\title{
Dispersion Engineered Radiative \& Guided-Wave Electromagnetic Structures for Efficient Wave Control
}

\author{
by \\ Mohamed K. Emara, B.Eng
}

A thesis submitted to the Faculty of Graduate and Postdoctoral Affairs in partial fulfillment of the requirements for the degree of

Master of Applied Science

in

Electrical \& Computer Engineering

Carleton University

Ottawa, Ontario

(C) 2018

Mohamed K. Emara

https://doi.org/10.22215/etdsw/2018.2835 


\section{Abstract}

Dispersion engineering is the process of controlling the phase response of an electromagnetic (EM) structure as a function of frequency, which in turn determines the propagation characteristics of EM waves in the structure. This thesis presents two novel dispersion engineered structures for controlling the propagation properties of EM waves for two different applications. The first structure is a low-profile dielectric choke ring for multipath mitigation for global navigation satellite systems (GNSS) applications. The proposed structure has a dual-band surface impedance resonance and it is designed by engineering the surface impedance to strategically introduce two resonant frequencies around the GNSS bands of L2 (1227.60 MHz) and L1 (1575.42 $\mathrm{MHz}$. This provides capacitive surface impedances around both bands simultaneously, required for efficient surface wave suppression for higher multipath rejection performance. To demonstrate the proposed principle of surface impedance engineering, an in-house choke ring prototype of the proposed design is tested with the widely used Dorne \& Margolin (DM) antenna on top of it. A high multipath rejection in both GNSS bands is experimentally confirmed with comparable performances to an otherwise bulky, heavy, and expensive conventional metallic choke ring. The proposed design further provides $58 \%$ reduction in height and $26 \%$ reduction in diameter using a low-cost FR4 dielectric material. The second structure is an amplitude-equalized group delay device, called a phaser, for analog signal processing (ASP) applications, implemented at microwave and millimeter-wave (mm-wave) frequencies. The proposed devices achieve a flat magnitude transmission while preserving their strong dispersive group delay response. The microwave phaser is realized using C-sections, and the mm-wave phaser is realized using micro-ring resonators implemented using half-mode substrate integrated waveguide (HMSIW) structures. Both types of phasers are successfully demonstrated using full-wave simulations, with experimental demonstration currently underway for the active configurations. 


\section{Acknowledgements}

My deepest gratitude and appreciation go to my supervisors Prof. Shulabh Gupta and Prof. Jim Wight. I would like to thank Prof. Wight for bringing in his unparalleled knowledge and experience whenever I faced challenges along the way. I would also like to thank Prof. Gupta for his encouragement, mentorship, and for always being available to advise and guide me. Prof. Gupta's tremendous energy and passion for research have inspired me to learn and grow which made my experience truly enjoyable. I would also like to thank my research group members, Muhammad Ali, Daniel King, Kimi Maheshwari, and Naquash Sheikh for their support and for giving me their feedback on my work during our weekly meetings.

I would also like to acknowledge the support I received from the engineering team at Tallysman Wireless Inc. I would like to thank Gyles Panther and Dr. Julien Hautcoeur for their guidance and mentorship. I would also like to thank Marc Brisebois, Micah Grinstead, and Chloe Xiao for their help with the fabrication processes.

I am also grateful to the thesis committee, Prof. Rony Amaya, Prof. Derek McNamara, Dr. Aldo Petosa, and Prof. Sreeraman Rajan, for taking the time to attend my defence and for providing me with constructive feedback on my work.

Special thanks to Nagui Mikhail from Carleton University for assisting me in the testing phase and for going above and beyond to help me finish my projects on time.

Finally, I wish to express my thanks to my family and friends for their continuous support. I am especially grateful to my parents, Karam and Hanaa, and my sisters, Merna and Maram, for their encouragement and for always believing in me. 


\section{Contents}

$\begin{array}{lll}\text { Abstract } & \text { ii }\end{array}$

$\begin{array}{ll}\text { Acknowledgements } & \text { iii }\end{array}$

Contents

List of Tables $\quad$ vii

List of Figures $\quad$ viii

List of Acronyms $\quad$ xiii

Nomenclature $\quad$ xv

1 Introduction 1

1.1 Dispersion Engineering . . . . . . . . . . . . . . . . 1

1.2 Thesis Objectives $\ldots \ldots \ldots \ldots \ldots \ldots \ldots \ldots$

1.3 Thesis Contributions . . . . . . . . . . . . . . . . . 3

1.4 Thesis Organization . . . . . . . . . . . . . . . . . . 4

2 Dual-Band Dielectric Choke Ring for GNSS Applications 6

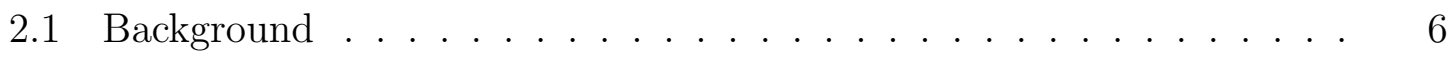

2.1.1 Global Navigation Satellite Systems . . . . . . . . . . . 6 
2.1 .2 Interference $\ldots \ldots \ldots \ldots \ldots \ldots \ldots \ldots \ldots$

2.2 Theory . . . . . . . . . . . . . . . . . . . . . 9

2.2 .1 Surface Waves . . . . . . . . . . . . . . . . . . . . . . 9

2.2.2 Perfect Electric Conductors . . . . . . . . . . . . . . . . 11

2.2 .3 Perfect Magnetic Conductors _. . . . . . . . . . . . . 12

2.2.4 Artificial Magnetic Conductors . . . . . . . . . . . . . . 12

2.2 .5 Choke Ring . . . . . . . . . . . . . . . . . . . . . 13

2.3 Literature Review . . . . . . . . . . . . . . . . . . . . . . 16

2.4 Proposed Design $\ldots \ldots \ldots \ldots$

2.4.1 Choke Ring Analyses . . . . . . . . . . . . . . . . . . . . . 19

2.4.2 Proposed Dielectric Choke Ring . . . . . . . . . . . . . . 21

2.4.3 Full-Wave Demonstration . . . . . . . . . . . . . . . . 24

2.4.4 Experimental Demonstration . . . . . . . . . . . . . . . 26

2.4 .5 Discussion . . . . . . . . . . . . . . . . . 36

2.4 .6 Conclusion . . . . . . . . . . . . . . . . . . . . . 38

3 Active Phasers for Analog Signal Processing Applications 39

3.1 Background . . . . . . . . . . . . . . . . . . . 39

3.2 Proposed Active Microwave C-Section . . . . . . . . . . . . . 40

3.2.1 Contra-directional Couplers based Phaser . . . . . . . . . . . 40

3.2 .2 Proposed Active Phaser . . . . . . . . . . . . . . . . 42

3.3 Proposed Active Millimeter-Wave Phaser . . . . . . . . . . . . 45

3.3.1 Co-directional Couplers based Phaser . . . . . . . . . . . 45

3.3.2 Proposed Active Phaser _. . . . . . . . . . . . . . . 49

3.4 Full-Wave Demonstration . . . . . . . . . . . . . . . . . 51 
3.4.1 Half-Mode Substrate-Integrated Waveguide Implementation 51

3.4.2 Single \& Cascaded Active Phasers . . . . . . . . . . . . . . . . 51

3.5 Experimental Demonstration . . . . . . . . . . . . . . 53

3.5.1 Design Prototypes . . . . . . . . . . . . . . 53

3.5.2 Testing . . . . . . . . . . . . . . . . . . 55

3.5.3 Results...................... 55

3.6 Conclusion . . . . . . . . . . . . . . . . . . . . 59

4 Conclusions \& Future Work $\quad 60$

4.1 Conclusions . . . . . . . . . . . . . . . . . . . . 60

4.2 Future Work . . . . . . . . . . . . . . . . . 61

4.2.1 Dual-Band Dielectric Choke Ring GNSS Applications . . . . . 61

4.2.2 Active Phasers for Analog Signal Processing Applications . . . 61

$\begin{array}{ll}\text { Appendices } & 72\end{array}$

$\begin{array}{ll}\text { A Dual-Band Choke Ring Drawing } & 73\end{array}$ 


\section{List of Tables}

Table 2.1 Choke ring corrugation depths and their effect on the surface impedance. . . . . . . . . . . . . . . . . 15 


\section{List of Figures}

Figure 1.1 EM properties of a corrugated metallic structure based on air and dielectric choke rings. a) Reflection phase, and b) surface impedance. 3

Figure 2.1 a) An illustration of direct LOS signals and reflected signals from an obstacle and ground reaching the antenna, and b) an illustration of the down-up ratio calculation. . . . . . . . . . . .

Figure 2.2 Illustration of Transverse Electric (TE) and Transverse Magnetic $(\mathrm{TM})$ surface waves on a plane. . . . . . . . . . . . .

Figure 2.3 Photograph of a commercial conventional metallic choke ring with dimensions $a=380 \mathrm{~mm}, d=63 \mathrm{~mm}, s=25 \mathrm{~mm}$ and $t=3 \mathrm{~mm}$. The cross-section of the choke ring is also shown below. . . . . . . .

Figure 2.4 FEM-HFSS choke ring model used to compute reflection phase and surface impedance. . . . . . . . . . . . . . . . .

Figure 2.5 Comparison of the EM properties of three choke ring configurations: a conventional choke ring, a choke ring with corrugation cavities filled with FR4, and a choke ring with corrugations cavities filled with Rogers 6010. a) Reflection phase, and b) surface impedance. . . . . .

Figure 2.6 Two electromagnetically equivalent dielectric choke rings. a) Multi-dielectric choke ring made by filling corrugation cavities with FR4 and Neltec NX9240. The dimensions of the structure required to achieve dual resonance at L2 and L1 are as follows: $a=280 \mathrm{~mm}$, $s=10 \mathrm{~mm}, d=29.2 \mathrm{~mm}$, and $t=3 \mathrm{~mm}$. b) Proposed grooved dielectric choke ring made by filling corrugation cavities with FR4 with air grooves. The dimensions of the structure required to achieve dual resonance at L2 and L1 are as follows: $a=280 \mathrm{~mm}, s=10 \mathrm{~mm}, d=$ $29.2 \mathrm{~mm}, d_{g}=15 \mathrm{~mm}, w=4 \mathrm{~mm}$, and $t=3 \mathrm{~mm} . \ldots \ldots$. . . 
Figure 2.7 Dual-band EM response of the multi-dielectric design and the proposed grooved dielectric design. a) Reflection phase, and b) surface impedance.

Figure 2.8 Surface impedance comparison between ungrooved and proposed grooved FR4 choke rings. . . . . . . . . . . . . . . .

Figure 2.9 Parametric study of the grooved-dielectric design to independently control two AMC points for dual-band surface suppression operation. a) Tuning the second frequency L1 by changing the depth of the FR4 grooves, and b) tuning the first frequency L2 by changing the height of the whole structure and the depth of the FR4 grooves. . . .

Figure 2.10 FEM-HFSS simulation models of the Dorne \& Margolin (DM) antenna in various configurations: a) stand-alone, b) on top of a conventional choke ring, and c) on top of the proposed dielectric choke ring. . . . . . . . . . . . . . . . . . .

Figure 2.11 FEM-HFSS simulated radiation patterns, down-up ratio, and axial ratio of the DM antenna in various configurations: standalone, on top of a conventional choke ring, and on top of the proposed dielectric choke ring at a) L2 and b) L1 frequency bands. All plots are at $\phi=0^{\circ}$ principal cut. . . . . . . . . . . . . . . .

Figure 2.12 FEM-HFSS model of the proposed dielectric choke ring implementation with copper posts replacing walls. . . . . . . . . .

Figure 2.13 a) Reflection phase of multiple number of copper posts replacing metallic walls. Number of posts replacing each ring, starting from the inner wall: Case $1(90,120,120,180,180$ [total = 600]), Case 2 (60, $60,60,60,60[$ total $=300])$, and Case $3(36,40,45,60,60$ [total $=$ 241]), and b) Case 2 before and after adjusting dimensions to get the phase response close to metallic walls. . . . . . . . . . .

Figure 2.14 Top and bottom views of the fabricated grooved dielectric choke ring with the DM antenna. . . . . . . . . . . . .

Figure 2.15 Testing the DM antenna in various configurations: a) standalone and b) on top of the conventional choke ring, using the Satimo system at Polytechnique Montreal, and c) on top of the proposed dielectric design in the anechoic chamber at Carleton University. . . . .

Figure 2.16 Experiment and FEM-HFSS simulation radiation patterns of the DM antenna at a) L2, and b) L1. . . . . . . . . . . . . . . 
Figure 2.17 Comparison between the experiment and FEM-HFSS simulation results of the radiation patterns of the proposed design at L2 and various other frequencies around the L2 band a) L2 (1227.60 MHz), b) $1250 \mathrm{MHz}$, c) $1260 \mathrm{MHz}$, and d) $1270 \mathrm{MHz}$. . . . . . . . . . . .

Figure 2.18 Comparison between the experiment and FEM-HFSS simulation results of the radiation patterns of the proposed design at L1 and various other frequencies around the L1 band a) $1560 \mathrm{MHz}$, b) L1 (1575.42 MHz), c) $1590 \mathrm{MHz}$, and d) $1600 \mathrm{MHz}$. . . . . . . . . . .

Figure 2.19 Comparison between the down-up ratio of the proposed design (experiment \& FEM-HFSS simulation) and the conventional metallic choke ring (experiment) at L2 and various other frequencies around the L2 band a) L2 (1227.60 MHz), b) $1250 \mathrm{MHz}$, c) $1260 \mathrm{MHz}$, and d) $1270 \mathrm{MHz} \ldots \ldots \ldots \ldots \ldots \ldots$

Figure 2.20 Comparison between the down-up ratio of the proposed design (experiment \& FEM-HFSS simulation) and the conventional metallic choke ring (experiment) at L1 and various other frequencies around the L1 band a) $1560 \mathrm{MHz}$, b) L1 (1575.42 MHz), c) $1590 \mathrm{MHz}$, and d) $1600 \mathrm{MHz} \ldots \ldots \ldots \ldots \ldots \ldots$

Figure 2.21 Measured axial ratio of a) the proposed design, and b) the conventional choke ring. . . . . . . . . . . . . . . . . . .

Figure 2.22 FEM-HFSS demonstration of effect of surface impedance on axial ratio. . . . . . . . . . . . . . . . . . .

Figure 3.1 a) Contra-directional coupler, and b) microwave C-section based on contra-directional coupler. . . . . . . . . . . . . . .

Figure 3.2 Proposed configuration of an amplitude-equalized microwave C-section, where the through and isolated ports of the corresponding coupler are connected through a load $G$. . . . . . . . . . . . .

Figure 3.3 The magnitude transmission and group delay responses of an active microwave C-section of Fig. 3.2 computed using (3.6). The responses of a passive C-section is also shown using dashed curves for comparison. The design parameters are: $k=0.71, \alpha \ell=0.15$ and the gain $G_{0}$ calculated using $(3.7) \ldots \ldots \ldots$

Figure 3.4 FEM-HFSS Simulated S-parameters and group delay response of an active $\mathrm{C}$-section implemented in stripline technology, for different values of Gain load, including the optimum value $G_{\mathrm{opt}}=0.17 \mathrm{~dB}$. 
Figure 3.5 Co-directional coupler with length, $l$ and gap, $g$. The codirectional coupling is $s_{1}$, and through is $s_{2}$. In an ideal co-directional coupler, contra-directional coupling, $s_{3}=0$, and reflection, $s_{4}=0$. . .

Figure 3.6 Phaser based on co-directional coupler formed by making a ring from one section of the coupler. . . . . . . . . . . . . . . . 46

Figure 3.7 Parametric study of the effect of ring length on the phaser response. a) Transmission as a function of frequency, and b) group delay as a function of frequency, with various ring lengths. . . . . . .

Figure 3.8 Parametric study of the effect of coupling coefficient on the phaser response. a) Transmission as a function of frequency, and b) group delay as a function of frequency, with various coupling coefficients. 47

Figure 3.9 Parametric study of the effect of attenuation constant on the phaser response. a) Transmission as a function of frequency, and b) group delay as a function of frequency, with various attenuation constants. . . . . . . . . . . . . . . . .

Figure 3.10 Proposed active phaser design involving adding a gain element inside the phaser ring to achieve a flat transmission response. . . . . .

Figure 3.11 Parametric study of the effect of gain on the phaser response of a lossy structure. a) Transmission as a function of frequency, and b) group delay as a function of frequency, with various gains integrated within the phaser ring. . . . . . . . . . . . . . . . . .

Figure 3.12 Phaser implementation using HMSIW structure. . . . . . . . . 51

Figure 3.13 Single HMSIW phaser: comparison of passive vs. active responses. a) Transmission as a function of frequency, and b) group delay as a function of frequency. . . . . . . . . . . . . . .

Figure 3.14 Cascaded HMSIW phaser: comparison of passive vs. active responses. a) Transmission as a function of frequency, and b) group delay as a function of frequency. . . . . . . . . . . . . .

Figure 3.15 Photograph of MACOM MAAM-011100 variable gain amplifier test board. . . . . . . . . . . . . . . . . . . . .

Figure 3.16 Photograph of the fabricated bandpass filter test board. . . . . 53

Figure 3.17 Photographs of the fabricated a) single and b) cascaded passive phasers with the bandpass filter at the input. . . . . . . . . . 
Figure 3.18 Photograph of the fabricated single active phaser with the amplifier integrated in the ring and the bandpass filter at the input. . . .

Figure 3.19 Bandpass filter results: a) transmission $\left(S_{21}\right)$, b) group delay, c) input return loss $\left(S_{11}\right)$, and d) output return loss $\left(S_{22}\right) \ldots \ldots 56$

Figure 3.20 Single passive phaser results: a) transmission $\left(S_{21}\right)$, b) group delay, c) input return loss $\left(S_{11}\right)$, and d) output return loss $\left(S_{22}\right)$. . .

Figure 3.21 Cascaded passive phaser results: a) transmission $\left(S_{21}\right)$, b) group delay, c) input return loss $\left(S_{11}\right)$, and d) output return loss $\left(S_{22}\right)$. . .

Figure A.1 Mechanical drawing of the manufactured dual-band dielectric choke ring . . . . . . . . . . . . . . . 


\section{List of Acronyms}

\begin{tabular}{|c|c|}
\hline $\mathrm{ADC}$ & analog-to-digital converter \\
\hline $\mathrm{AMC}$ & artificial magnetic conductor \\
\hline ASP & analog signal processing \\
\hline BNS & Beido navigation satellite system \\
\hline $\mathrm{DAC}$ & digital-to-analog converter \\
\hline $\mathrm{DM}$ & Dorne \& Margolin \\
\hline DCMA & dispersion code multiple access \\
\hline DSP & digital signal processing \\
\hline DU ratio & down-up ratio \\
\hline DUT & device-under-test \\
\hline $\mathrm{EBG}$ & electromagnetic bandgap \\
\hline EM & electromagnetic \\
\hline FEM & finite element moment \\
\hline FDTD & finite-difference time-domain \\
\hline GA & genetic algorithm \\
\hline GLONASS & global navigation satellite system \\
\hline GNSS & global navigation satellite systems \\
\hline GPS & global positioning system \\
\hline HFSS & high frequency structure simulator \\
\hline HIS & high impedance surface \\
\hline HMSIW & half-mode substrate integrated waveguide \\
\hline PBG & photonic bandgap \\
\hline $\mathrm{PCB}$ & printed circuit board \\
\hline PDM & perfect dispersive medium \\
\hline
\end{tabular}




$\begin{array}{ll}\text { PEC } & \text { perfect electric conductor } \\ \text { PMC } & \text { perfect magnetic conductor } \\ \text { PML } & \text { perfectly matched layer } \\ \text { LHCP } & \text { left-hand circularly polarized } \\ \text { LOS } & \text { line-of-sight } \\ \text { TE } & \text { transverse electric } \\ \text { TEM } & \text { transverse electromagnetic } \\ \text { TM } & \text { transverse magnetic } \\ \text { RHCP } & \text { right-hand circularly polarized } \\ \text { RSW } & \text { reduced surface wave } \\ \text { SIW } & \text { substrate integrated waveguide } \\ \text { R-ASP } & \text { real-time analog signal processing } \\ \text { RTFT } & \text { real-time Fourier transformation } \\ \text { VGA } & \text { variable gain amplifier } \\ \text { VNA } & \text { vector network analyzer }\end{array}$




\section{Nomenclature}

c

$\omega$

$f$

$\lambda$

$\beta, k$

$\epsilon$

$\epsilon_{0}$

$\epsilon_{r}$

$\mu$

$\mu_{0}$

$\mu_{r}$

$\alpha$

$H$

E

$J$

$\sigma$

$\delta$

$\Gamma$

$\eta$

$Z_{S}$

$\kappa$

$\gamma$

$\tau_{g}$ speed of light in air or vacuum, $3 \times 10^{8} \mathrm{~m} / \mathrm{s}$

angular frequency, $\mathrm{rad} / \mathrm{s}$

frequency, $\mathrm{Hz}$

wavelength, $\mathrm{m}$

phase constant, $\mathrm{rad} / \mathrm{m}$

electric permittivity, $\mathrm{F} / \mathrm{m}$

electric permittivity of free space, $8.854 \times 10^{-12} \mathrm{~F} / \mathrm{m}$

relative electric permittivity

magnetic permeability, $\mathrm{H} / \mathrm{m}$

magnetic permeability of free space, $4 \pi \times 10^{-7} \mathrm{H} / \mathrm{m}$

relative magnetic permeability

attenuation constant, $\mathrm{Np} / \mathrm{m}$

magnetic field intensity, $\mathrm{A} / \mathrm{m}$

electric field intensity, $\mathrm{V} / \mathrm{m}$

electric current density, $\mathrm{A} / \mathrm{m}^{2}$

electric conductivity, $\mathrm{S} / \mathrm{m}$

skin depth, m

reflection coefficient

wave impedance, $\Omega$

surface impedance, $\Omega$

voltage coupling coefficient

propagation constant

group delay, s 


\section{Chapter 1}

\section{Introduction}

\section{$1.1 \quad$ Dispersion Engineering}

Dispersion of a given electromagnetic (EM) structure describes the frequency-dependent phase velocity, and by extension the phase constant, of an EM wave inside the structure [1]. Media exhibiting this phenomenon are called dispersive media. Dispersion is the property that causes white light to split into a spectrum by the popular dispersion prism [2]. While air is non-dispersive and allows all frequencies to propagate with the same velocity (i.e. $c$, speed of light in air or vacuum), a prism is dispersive so different frequencies have different phase velocities and different angles of refraction inside the prism. This causes different frequencies to separate in space, hence splitting white light to its constituent colors, some of which are visible. Another example of a dispersive structure is a waveguide in which the structure's geometry determines the propagation of EM waves inside, for example by imposing a cut-off frequency below which no propagation can occur [3].

From a mathematical point of view, dispersion can be described by the relationship between the phase constant, frequency, and the phase velocity. For a non-dispersive medium such as air, the phase constant can be described using the following equation:

$$
\beta(\omega)=\frac{\omega}{c}
$$

where $\beta$ is the phase constant in $\mathrm{rad} / \mathrm{m}, \omega$ is the angular frequency in $\mathrm{rad} / \mathrm{s}$ and $c=3 \times 10^{8} \mathrm{~m} / \mathrm{s}$ is the speed of light in air or vacuum. For a dispersive medium, the phase velocity is frequency-dependent, so the equation becomes:

$$
\beta(\omega)=\frac{\omega}{u_{p}(\omega)}
$$


where $u_{p}$ is the frequency-dependent phase velocity. From the previous equations, it is seen that the phase constant is a linear function of frequency for a non-dispersive medium, but not necessarily for a dispersive medium.

Group delay is the time delay of the amplitude of a signal as it passes through a structure, and it is defined as the derivative of phase with respect to frequency, as a function of frequency as follows:

$$
\tau_{g}(\omega)=-\frac{d \phi(\omega)}{d \omega}
$$

For a non-dispersive medium with the phase constant being a linear function of frequency, group delay is constant. For dispersive media, group delay could have a non-constant profile.

Another quantity of interest and related to material dispersion is surface impedance, which is the ratio of the tangential electric field to the tangential magnetic field on a plane interface [4]. Surface impedance determines the propagation characteristics of EM waves on the surface (called surface waves). For any given surface, a relationship can be found between surface impedance and the reflection phase of a plane wave at the surface. For example, a corrugated metallic structure such as the choke ring (to be discussed in more detail in Sec. 2.2.5 and Sec. 2.4.1) has a linear relationship between reflection phase and frequency with air as shown in Fig. 1.1(a). On the other hand, the reflection response is highly non-linear when a dielectric is used instead of air, thus making the phase response strongly dispersive. The zero reflection phase occurs at the frequency where the depth of the corrugations is a quarter-wavelength. The same phenomenon can be described in terms of surface impedance, where zero reflection phase corresponds to an open circuit. Fig. 1.1(b) shows the corresponding surface impedance as a function of frequency where a surface impedance resonance occurs at the same frequency as the zero reflection phase. The surface impedance is inductive on one side of the resonance and capacitive on the other side.

Dispersion engineering is the process of controlling the phase response of an EM structure as a function of frequency, which in turn determines the propagation characteristics of EM waves in the structure. In this thesis, the concept of dispersion engineering is used for two applications:

1. Surface impedance engineering of a ground plane to control its interaction with an antenna for radiative EM applications related to multipath mitigation for global navigation satellite systems (GNSS) applications.

2. Group delay engineering of a waveguide structure to control the dispersion characteristics of guided EM waves for analog signal processing (ASP) applications. 


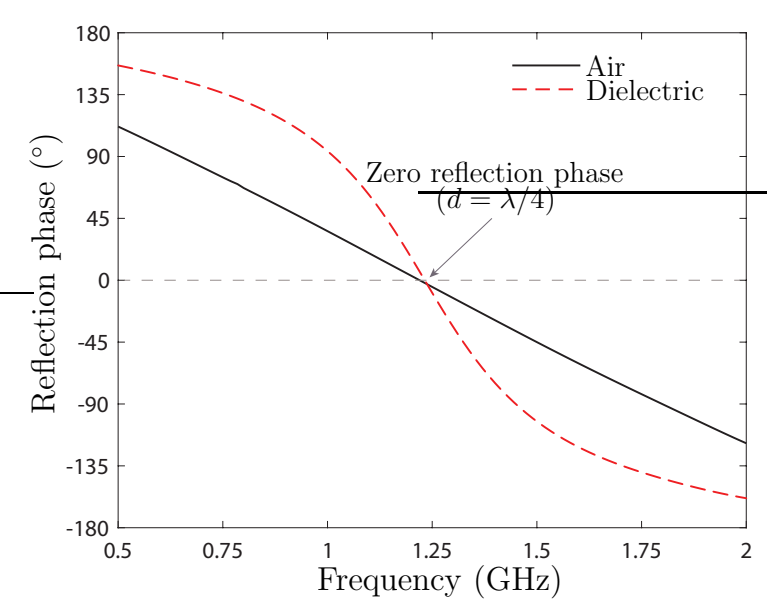

(a)

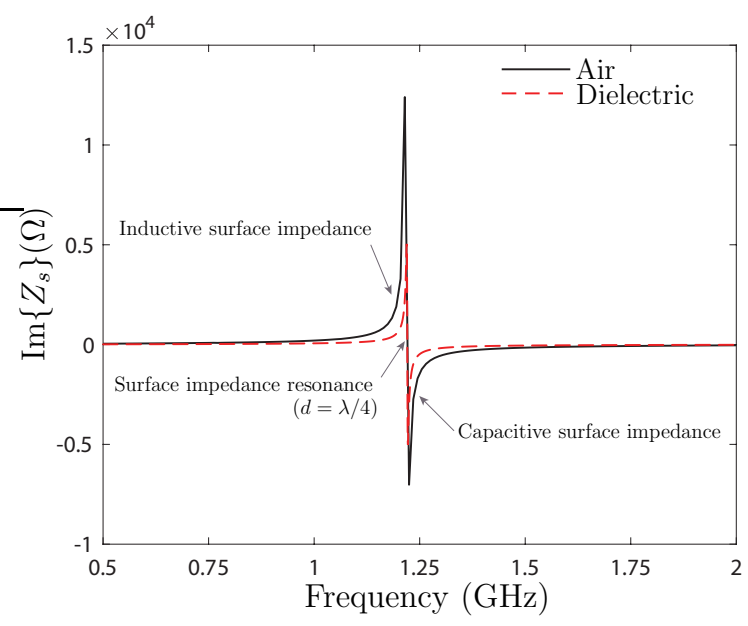

(b)

Figure 1.1: EM properties of a corrugated metallic structure based on air and dielectric choke rings. a) Reflection phase, and b) surface impedance.

\subsection{Thesis Objectives}

The thesis has two objectives:

1. Designing a low-profile low-cost ground plane for multipath mitigation for GNSS applications with comparable performance to the conventional choke ring ground plane.

2. Designing amplitude-equalized dispersive group delay devices, called phasers, for microwave and millimeter-wave (mm-wave) ASP applications.

\subsection{Thesis Contributions}

The work done in this thesis has led to two contributions:

1. A novel low-profile dual-band grooved-dielectric choke ring for multipath mitigation for GNSS applications. The proposed dielectric choke ring exhibits dual-band surface wave suppression characteristics at the GNSS bands of L2 $(1227.60 \mathrm{MHz})$ and L1 (1575.42 MHz), hence providing good multipath mitigation performance at the two bands simultaneously. This work has led to two paper submissions [5], [6]:

- "Surface Impedance Engineered Low-Profile Dual-Band Grooved-Dielectric Choke Ring for GNSS Applications" has been submitted to IEEE Transactions on Antennas and Propagation on April $6^{\text {th }}, 2018$. 
- "A Low-Profile Dual-Band Tunable AMC Structure for GNSS Antennas and its Performance Trade-Offs" has been submitted to the $18^{\text {th }}$ International Symposium on Antenna Technology and Applied Electromagnetics (ANTEM) conference to take place in August 2018.

2. Amplitude equalized single and cascaded active phasers based on co- and contradirectional couplers for microwave and mm-wave ASP applications. This work has led to one publication [7], and one paper submission [8]:

- "Active Phasers based on Co-directional Couplers for Millimeter-wave Analog Signal Processing" has been submitted to the $18^{\text {th }}$ International Symposium on Antenna Technology and Applied Electromagnetics (ANTEM) conference to take place in August 2018.

- "Amplitude-Equalized Microwave Phasers" has been accepted and presented in the International Applied Computational Electromagnetics Society (ACES) Symposium in March 2018.

\subsection{Thesis Organization}

The thesis is structured as follows:

- Chapter 1 provides an introduction to dispersion and how it relates to the dispersion engineered structures presented in the thesis. The chapter then discusses the thesis objectives, contributions, and organization.

- Chapter 2 deals with the first goal of the thesis and begins by providing a background of multipath interference as it relates to GNSS and the theory of surface waves and their propagation on corrugated ground planes. The chapter then gives a detailed account of the analysis and design process that led to the design of the dual-band dielectric choke ring and the fabrication of the prototype. Next, the chapter presents experimental demonstration of the characteristics of the dielectric choke ring tested with the Dorne \& Margolin (DM) antenna. The chapter then presents a discussion of the ground plane performance as it compares to the conventional choke ring. Finally, a conclusion of the work is provided.

- Chapter 3 deals with the second goal of the thesis and begins by providing a background of ASP and the need for dispersive group delay devices. The chapter then breaks into two sections. The first section deals with the operation of the active "microwave" phaser and its full-wave demonstration. The second section deals with the operation of the active "mm-wave" phaser, its full-wave demonstration, and the fabrication efforts for experimental demonstration. Finally, a conclusion of the work is provided. 
- Chapter 4 provides the thesis conclusion and a discussion of the future work related to the proposed dielectric choke ring and the proposed active phasers. 


\section{Chapter 2}

\section{Dual-Band Dielectric Choke Ring for GNSS Applications}

\subsection{Background}

\subsubsection{Global Navigation Satellite Systems}

Modern civilian and military technologies are highly reliant on the global navigation satellite systems (GNSS). GNSS includes the Global Positioning System (GPS) [9], the Global Navigation Satellite System (GLONASS) [10], Galileo [11], and BeiDou Navigation Satellite System (BNS). GPS is owned and operated by the Department of Defense of the United States and it is fully operational. GLONASS is Russia's constellation and it is also fully operational. Galileo, Europe's new constellation, operated by the European Space Agency, is expected to be fully operational by 2020 . BeiDou, China's constellation, is regionally operational and expected to provide global coverage by 2020. GNSS signals are right-hand circularly polarized (RHCP), so ideally a GNSS antenna must be RHCP with good cross-polarization (i.e. left-hand circular polarization (LHCP)) rejection. Of all GNSS signals, the GPS L2 $\left(f_{c}=1227.60 \mathrm{MHz}\right.$ and $24 \mathrm{MHz}$ bandwidth) and $\mathrm{L} 1\left(f_{c}=1575.42 \mathrm{MHz}\right.$ and $24 \mathrm{MHz}$ bandwidth) signals are the most popular. Other GNSS signals fall in frequency bands around L2 and L1, for example, the Galileo E1 signal has the same center frequency as L1 and bandwidth of $36 \mathrm{MHz}$. More information about GNSS signal plans can be found in [12].

With the introduction of new technologies such as automated vehicles, it is crucial for navigation systems to be accurate, precise, and reliable. Since the GNSS signals have low power by the time they arrive at the GNSS receiver, the presence of radio frequency signals from other communications systems can "drown" GNSS signals. Other major threats to GNSS reliability include jamming, spoofing, and multipath interference [13]. In order for GNSS measurements to be done with sub-centimeter 
level of accuracy and precision, the GNSS receiver must be resilient to various types of interference threats. The focus of this thesis is multipath interference, which is considered to be a major obstacle to high precision measurements at the antenna level of the GNSS receiver [14].

\subsubsection{Interference}

Interference between the direct satellite signal and other signals poses a threat to the integrity of GNSS. This section is dedicated to discussing various sources of interference, with a focus on multipath.

\subsubsection{Natural Sources of Interference}

Natural interference is caused by the earth's atmosphere. The ionosphere can introduce delays on the propagation time of the signal through it. The amount of delay is dependent on the ionosphere's total electron content, which is not constant [15]. Such a delay can be accounted for by using models of the ionosphere for single-frequency measurements. For dual-frequency measurements, the delay can be corrected without prior knowledge of the ionosphere. Theoretical treatment of ionospheric error correction using two frequencies can be found in [16].

\subsubsection{Artificial Interference}

GNSS signals have low power and are prone to be "drowned" by other communications signals. Artificial interference can be broken down into two categories: intentional and unintentional interference. Examples of unintentional interfering signals are harmonics and intermodulation products generated by transmitters of other signals. For example, the uplink frequency band of Inmarsat signals is $1626.5-1660.5 \mathrm{MHz}$ [17]. Two Inmarsat signals at $1630 \mathrm{MHz}$ and $1660 \mathrm{MHz}$, generate a third intermodulation product at $1600 \mathrm{MHz}$, which is in the G1 frequency band of GLONASS. This interference can be mitigated with proper filtering in the RF front-end.

Intentional interference which adds noise to the GNSS signals is called jamming and it causes the receiver to lose track of the satellite, and so poses a threat to GNSS signal availability. Intentional interference intended to introduce errors in position and timing by transmitting GNSS-like signals is called spoofing [13], and it tricks the receiver into tracking the spoofing signal instead of the satellite. Spoofing can be mitigated with sophisticated digital signal processing techniques [18], or antenna solutions [19], [20]. 


\subsubsection{Multipath Interference}

Multipath interference is a major source of performance degradation in GNSS antennas. Multipath is an example of self-interference which occurs when the antenna receives reflected and diffracted satellite signals in addition to the direct line-of-sight (LOS) signal [13]. Reflected signals can occur from structures in close proximity to the antenna, this is a problem especially in areas with high-rise buildings. Fig. 2.1(a) shows an illustration of direct LOS and multipath signals reaching an antenna. GNSS signals are RHCP and become LHCP with odd number of reflections. A parameter called "down-up ratio" (DU ratio, for short) is used to quantify the antenna rejection of a LHCP signal below horizon relative to a RHCP above horizon at the same angle [21], [22] as shown in Fig. 2.1(b). The DU ratio is calculated for every elevation angle, $\theta$, using the following equation:

$$
\mathrm{DU}(\theta)[\mathrm{dB}]=G_{\mathrm{LHCP}}(-\theta)[\mathrm{dB}]-G_{\mathrm{RHCP}}(+\theta)[\mathrm{dB}]
$$

The lower the DU ratio, the better the rejection of LHCP multipath signals arriving from underneath the antenna.

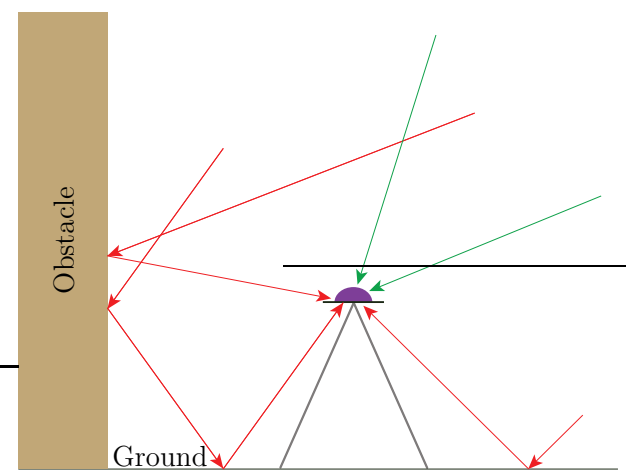

(a)

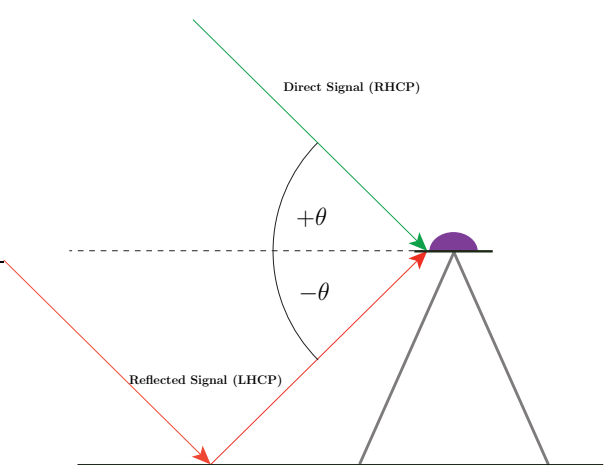

(b)

Figure 2.1: a) An illustration of direct LOS signals and reflected signals from an obstacle and ground reaching the antenna, and b) an illustration of the down-up ratio calculation.

Receiving replicas of satellite signals introduces errors in the carrier phase measurements, which results in pseudorange measurement errors [23]. Since the majority of multipath signals arrive from underneath the antenna or at angles of low elevation (i.e. close to the horizon), multipath signals can be rejected by designing an antenna having a sharp gain roll-off at horizon and low back and side lobes [24], [25]. For this purpose, antennas are usually supplemented with ground planes. Choke ring ground planes are typically used with reference antennas to mitigate the effects of multipath interference by blocking the propagation of surface waves generated by multipath signals close to the antenna, and thus reducing side lobes [24], [26]. 


\subsection{Theory}

\subsubsection{Surface Waves}

Understanding the behavior of surface waves is important in designing electromagnetic structures for multipath rejection. Multipath signals usually arrive close to the horizon from reflectors around the antenna and so can reach the antenna in the form of surface waves (also referred to as surface currents) on the ground plane.

Surface waves exist at the interface between two media, and while they are bound to the interface and have a propagation constant along the surface, they decay exponentially away from the surface [27]. Surface waves can propagate on conducting surfaces and when a finite conducting surface is used as an antenna ground plane, surface waves can radiate at the edges (a property known as edge diffraction [28]) and distort the radiation pattern of the antenna. Surface waves can also cause interference with other radiation patterns if multiple antennas share the same ground plane [29]. Several techniques are known in literature to suppress the propagation of surface wave. For example covering a conducting surface with small bumps [30], and using corrugated metallic structures [31], [32], [33], such as the choke ring [25]. The most recent development in surface wave suppression is the introduction of a thin printed circuit board (PCB) structure known as the mushroom structure [34]. Analysis of surface waves on plane interfaces can be found in [27], [35], and on corrugated surfaces in [27], [26].

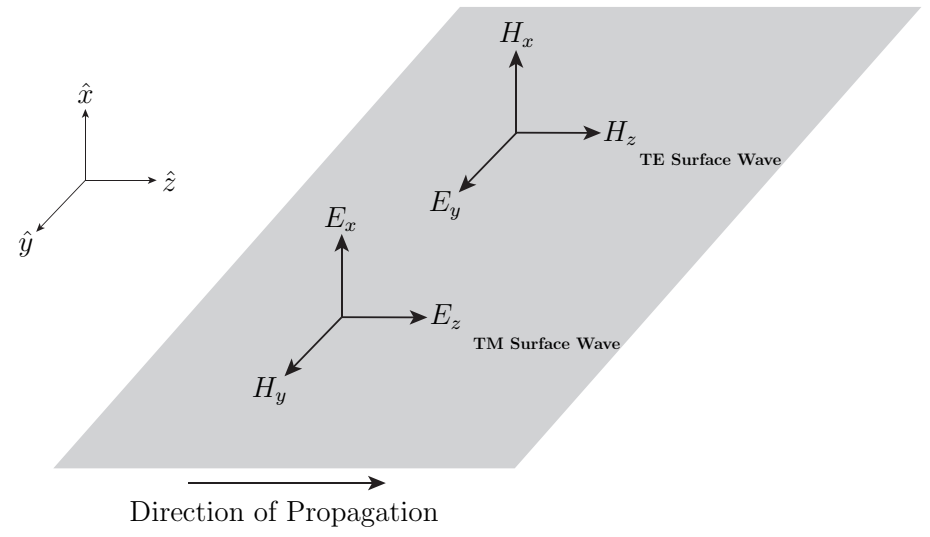

Figure 2.2: Illustration of Transverse Electric (TE) and Transverse Magnetic (TM) surface waves on a plane.

Two types of surface waves can exist along a plane, Transverse Magnetic (TM) polarized waves and Transverse Electric (TE) polarized waves, as shown in Fig. 2.2. Both types of waves will be analyzed here. The fields of a TM polarized wave can be represented in phasor form (and excluding time dependance $e^{j \omega t}[3]$ ) as follows: 


$$
\begin{aligned}
H_{y} & =\left(H_{y o} e^{-\alpha x} e^{-j k_{z} z}\right) \hat{y} \\
E_{z} & =\left(E_{z o} e^{-\alpha x} e^{-j k_{z} z}\right) \hat{z} \\
E_{x} & =\left(E_{x o} e^{-\alpha x} e^{-j k_{z} z}\right) \hat{x}
\end{aligned}
$$

where $H_{y o}, E_{z o}$, and $E_{x o}$ are the field amplitudes, $\alpha$ is the attenuation constant in the axis perpendicular to the surface (+x-direction), and $k_{z}$ is the phase constant in the direction of propagation (+z-direction).

The E- and H-fields are related by Maxwell's equation (in phasor form):

$$
\nabla \times H=J+j \omega \epsilon_{0} E
$$

In this analysis, the surface wave exists on an air-material interface and decays into air, therefore the conduction current can be neglected (i.e. $J \approx 0$ ), and the equation simplifies to $\nabla \times H=j \omega \epsilon_{0} E$. Taking the curl of the H-field yields:

$$
\nabla \times H=\left(j k_{z} H_{y o} e^{-\alpha x} e^{-j k_{z} z}\right) \hat{x}+\left(-\alpha H_{y o} e^{-\alpha x} e^{-j k_{z} z}\right) \hat{z}
$$

Equating this to the $j \omega \epsilon_{0} E$ results in the following equation:

$$
\begin{aligned}
& \left(j k_{z} H_{y o} e^{-\alpha x} e^{-j k_{z} z}\right) \hat{x}+\left(-\alpha H_{y o} e^{-\alpha x} e^{-j k_{z} z}\right) \hat{z} \\
= & \left(j \omega \epsilon_{0} E_{x o} e^{-\alpha x} e^{-j k_{z} z}\right) \hat{x}+\left(j \omega \epsilon_{0} E_{z o} e^{-\alpha x} e^{-j k_{x} x}\right) \hat{z}
\end{aligned}
$$

Using this equation, the amplitudes of the E-field components can be related to the magnetic field as:

$$
\begin{aligned}
& E_{x o}=\frac{k_{z}}{\omega \epsilon_{0}} H_{y o} \\
& E_{z o}=-\frac{\alpha}{j \omega \epsilon_{0}} H_{y o}
\end{aligned}
$$

The surface impedance can then be defined as:

$$
Z_{S}(\mathrm{TM})=\frac{E_{z o}}{H_{y o}}=-\frac{\alpha}{j \omega \epsilon_{0}}=\frac{j \alpha}{\omega \epsilon_{0}}
$$

A TE wave on the same surface has the following field components: 


$$
\begin{aligned}
& E_{y}=\left(E_{y o} e^{-\alpha x} e^{-j k_{z} z}\right) \hat{y} \\
& H_{z}=\left(H_{z o} e^{-\alpha x} e^{-j k_{z} z}\right) \hat{z} \\
& H_{x}=\left(H_{x o} e^{-\alpha x} e^{-j k_{z} z}\right) \hat{x}
\end{aligned}
$$

Applying the previous analysis to the TE surface wave yields the following surface impedance:

$$
Z_{S}(\mathrm{TE})=-\frac{E_{y o}}{H_{z o}}=\frac{-j \omega \mu_{0}}{\alpha}
$$

The previous analysis has shown that a TM surface wave requires a surface with inductive surface impedance, while a TE surface wave requires a surface with capacitive surface impedance. Therefore the surface impedance of an arbitrary structure will reveal whether it supports either types of surface waves. In addition, the surface impedance of a conducting plane was derived in [34]:

$$
Z_{S}=\frac{1+j}{\sigma \delta}
$$

where $\sigma$ is the conductivity and $\delta$ is the skin depth.

Based on the surface impedance of a conducting plane, one can conclude that such a plane supports TM surface waves but not TE surface waves (which is expected based on the boundary condition $E_{t}=0$ ). This explains why a conducting surface used as an antenna ground plane does not provide a good shield to low-elevation multipath signals since these signals can propagate on the ground plane and reach the antenna. A surface-wave-suppressing structure must effectively block the propagation of both TM and TE surface waves within the required frequency band to achieve multipath rejection.

\subsubsection{Perfect Electric Conductors}

Metals, such as copper, are usually treated as perfect electric conductors (PECs), meaning they are assumed to be lossless with conductivity $\sigma \approx \infty$. As such, no fields can exist inside a PEC (as opposed to conductors with finite conductivity which have a skin depth through which fields can exist). Applying boundary equations to a PEC results in boundary conditions that enforce no tangential electric fields (i.e. $E_{t}=0$ ) and non-zero tangential magnetic field (i.e. $H_{t} \neq 0$ ) [36].

Based on the analysis of plane waves [36], a PEC has wave impedance of $\eta=0$ and a reflection coefficient of $\Gamma=-1=1 \angle \pm 180^{\circ}$. Due to these properties, a PEC 
can be considered analogous to a short circuit $(V=0$ and $I \neq 0)$. Because of their unity reflection coefficient, good conductors are used as ground planes for antennas to improve antenna gain [37]. However due to their boundary conditions, a separation of $\lambda / 4$ between the antenna and the conductor is needed in order to have reflected fields add in-phase at the antenna for optimal radiation efficiency [34]. Good conductor ground planes partially shield the antenna from signals below horizon, but have the disadvantage of supporting surface wave propagation as was shown in Sec. 2.2.1 and so suffer from significant edge diffraction which distorts the radiation pattern [14]. Surface waves also cause mutual coupling between antennas sharing the same ground plane [29], [34].

\subsubsection{Perfect Magnetic Conductors}

Perfect magnetic conductors (PMCs) do not exist in nature, but can be artificially designed with narrow bandwidths (these structures are called artificial magnetic conductors or AMC). Just like a PEC, there are no fields inside a PMC, but a fictitious magnetic current density can exist on the surface. Applying boundary equations to this theoretical surface results in boundary conditions that enforce no tangential magnetic fields (i.e. $H_{t}=0$ ) and non-zero tangential electric field (i.e. $E_{t} \neq 0$ ) due to the theoretical magnetic current density.

While a PEC is analogous to a short-circuit, a PMC is analogous to an opencircuit $(V=\infty$ and $I=0)$. Using this analogy, the reflection phase of a PMC is $\Gamma=1 \angle 0^{\circ}$ and its surface impedance is infinite. PMCs can be used as ground planes for antennas without the quarter-wave separation imposed by a PEC since a PMC does not short out electric current [34].

\subsubsection{Artificial Magnetic Conductors}

Artificial magnetic conductors (AMCs) are periodic structures that emulate the boundary conditions and reflection characteristics of a PMC. An AMC structure exhibits the aforementioned properties at a single frequency with a bandwidth, defined as the range of frequencies over which the reflection phase is in the range of $\pm 90^{\circ}$. An example of an AMC structure is the mushroom-like structure invented by Sievenpiper [34] [38]. This structure consists of via-ground periodic metallic patches on top of a PEC-backed substrate, whereby the patches function as LC resonant circuits. Due to its in-phase reflection characteristics, the mushroom structure is a high impedance surface (HIS). The mushroom structure also suppresses the propagation of surface waves in a forbidden frequency band and hence can be referred to as an electromagnetic band gap (EBG) structure. At optical frequencies, a structure with a forbidden bandgap is called a photonic band gap (PBG) structure [39]. 
The in-phase reflection and surface wave suppression characteristics of a mushroomlike EBG and a uniplanar compact EBG [40] (similar the mushroom structure but with no vias to ground) were analyzed in [41]. A finite-difference time-domain (FDTD) model was used to analyze the in-phase behaviour where a single EBG cell was simulated with periodic boundary conditions (PBC) on four sides and a perfectly matched layer (PML) on the top to absorb the reflected wave. Oblique and normally incident waves were launched towards the surface and their reflection phase was measured. To analyze the surface wave suppression characteristics, the transmission through a suspended stripline passing over the EBG surface was measured. It was found that the $\pm 90^{\circ}$ phase reflection frequency region is not the same as the surface wave bandgap for the mushroom-like EBG for a normally incident wave, but for an oblique wave, the two bands match. For the uniplanar compact EBG, the in-phase reflection and surface wave bandgap matched for both normal and oblique incident waves. The paper has shown that the in-phase frequency of the mushroomlike structure is dependent on the polarization and angle of incidence of the wave. The in-phase reflection band and surface wave bandgap will only match if the polarization and incidence of the wave used in the stripline was used to measure the reflection phase.

Due to their in-phase reflection property, AMC structures can be used as ground planes for antennas without the need for the quarter-wavelength separation imposed by a PEC, resulting in low-profile designs. A few antenna designs that use an AMC or EBG structure as a ground plane include: a dual-band GPS patch antenna on a dual-band fractal EBG structure [42], a dual-band monopole antenna on a dual-band AMC with a cross shaped slot for zero reflection phase tuning [43], and a dual-band crossed asymmetric dipole antenna on a dual-band tunable AMC with slits in the mushroom patch for dual-frequency operation [44]. AMCs have also been used for antenna beam shaping and gain enhancement [45], [46].

Other developments in AMC and EBG technology include: a dual-band tunable AMC structure designed using periodic metallic loops loaded with reactive elements on a grounded substrate [47], a dual-band AMC structure designed using a capacitive grid on a grounded substrate [48], a novel technique for designing AMC surfaces using FDTD combined with genetic algorithm (GA) optimizations [49], a compact EBG designed using reverse split rings [50], and a dual-band tunable AMC composed of dual-layer mushroom patches [51].

\subsubsection{Choke Ring}

A choke ring is a corrugated metallic structure as shown in Fig. 2.3. It consists of several concentric metallic rings on a metallic base, with the antenna in the center. The choke ring is the standard ground plane used for surveying and reference antennas due to its ability to suppress surface waves on the ground plane. Surface wave suppression 
reduces antenna side and back lobes, and hence multipath signals arriving close to the horizon and from underneath the antenna are rejected. While conventional choke rings like the one shown in Fig. 2.3 provide excellent multipath mitigation at their design frequencies, they have the disadvantage of being bulky, heavy, and expensive.

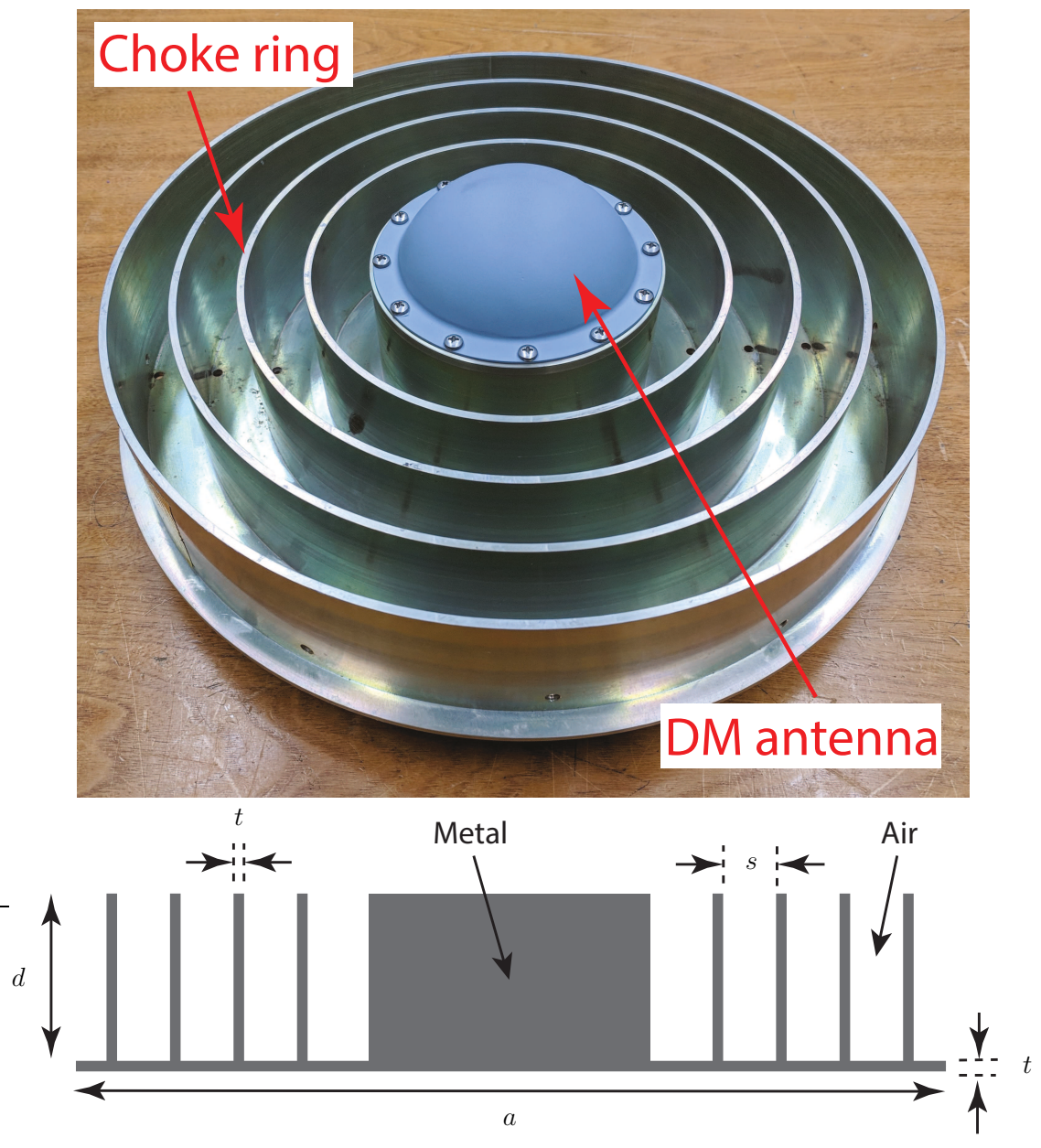

Figure 2.3: Photograph of a commercial conventional metallic choke ring with dimensions $a=380 \mathrm{~mm}, d=63 \mathrm{~mm}, s=25 \mathrm{~mm}$ and $t=3 \mathrm{~mm}$. The cross-section of the choke ring is also shown below.

The operation of the choke ring can be understood from the interaction of the choke ring with the two types of surface waves (TE \& TM) discussed in Sec. 2.2.1. This section will demonstrate that the choke ring suppresses the propagation of both types of surface waves (TE \& TM) in a certain frequency bandwidth specified by the depth of the corrugations. For a choke ring, the top surface of the metallic walls effectively suppresses the propagation of TE surface waves. On the other hand, TM surface waves are supported by metallic ground planes and so the ground plane must be engineered to block the propagation of TM surface waves for complete surface wave suppression.

The openings at the top of the choke ring have a surface impedance based on the 
depth, $d$, of the corrugations and can be calculated as follows [26]:

$$
Z_{S}=Z_{0} \frac{Z_{\text {bottom }}+j Z_{0} \tan k d}{Z_{0}+j Z_{\text {bottom }} \tan k d}
$$

where $Z_{0}=377 \Omega$ is the intrinsic impedance of air or free space, and $k=\frac{2 \pi}{\lambda}$ is the phase constant. Since the choke ring openings are shorted on the bottom, $Z_{\text {bottom }}=$ $0 \Omega$, and the equation can be simplified to [26]:

$$
Z_{S}=j Z_{0} \tan k d
$$

Note that for a corrugation depth of $d=\lambda / 4$ at the design frequency $\omega_{0}, Z_{S}\left(\omega_{0}\right)=$ $j(377)(\tan \pi / 2)=j(\infty)$ and the reflection coefficient at the surface is $\Gamma\left(\omega_{0}\right)=1 \angle 0^{\circ}$. Thus a quarter-wavelength deep corrugated surface has very high surface impedance, and acts as a PMC at $\omega_{0}$. As was discussed in Sec. 2.2.4, structures designed to have zero reflection phase are called AMCs. Based on its high surface impedance and zero reflection phase, the choke ring is an AMC and a high impedance surface (HIS) at the design frequency set by the quarter-wavelength depth of the corrugations [34].

Next, the propagation of a TM surface will be investigated for various corrugation depths. In Sec. 2.2.1, it was demonstrated that a TM surface wave requires a positive reactive surface impedance to propagate. Since (2.12) has a "tan" expression for the surface impedance of the corrugation opening, it will change polarity depending on the corrugation depth.

Table 2.1 shows three cases for corrugation depths and their corresponding surface impedances. A corrugation depth of $d=0$ corresponds to a flat metal sheet, therefore it supports the propagation of surface waves. For a corrugation depth $0<d<\lambda / 4$, the surface impedance is inductive, therefore TM surface waves are supported. For a corrugation depth $\lambda / 4<d<\lambda / 2$, the surface impedance is capacitive, thereby suppressing the propagation of TM surface waves, as desired in the choke ring. Naturally, the TM surface wave suppression is stronger as the surface impedance becomes more capacitive [37] (i.e. closer to $\lambda / 4$ ). It is important to note that for all corrugation depths, TE surface waves cannot propagate due to the boundary conditions at the top surface of the metallic walls.

Table 2.1: Choke ring corrugation depths and their effect on the surface impedance.

\begin{tabular}{|l|l|c|}
\hline Corrugation Depth, $d$ & Surface Impedance, $Z_{s}$ & $\begin{array}{c}\text { TM Surface Wave } \\
\text { Propagation }\end{array}$ \\
\hline$d=0$ & $Z_{s}=0$ & Yes \\
\hline $0<d<\lambda / 4$ & $Z_{s}=+j X$ & Yes \\
\hline$\lambda / 4<d<\lambda / 2$ & $Z_{s}=-j X$ & No \\
\hline
\end{tabular}

Based on these results, it is concluded that a corrugated structure with corrugation 
depth $\lambda / 4<d<\lambda / 2$ has a capacitive surface impedance and blocks the propagation of all surface waves, required for multipath rejection applications. It is also concluded that the surface wave suppression property of choke rings is wideband, with better performance closer to $\lambda / 4$. For GNSS applications where L2 $(1227.60 \mathrm{MHz})$ and L1 $(1575.42 \mathrm{MHz})$ are the frequencies of interest, the corrugation depth must be chosen such that $\lambda_{L 2} / 4<d<\lambda_{L 1} / 2(61 \mathrm{~mm}<d<95 \mathrm{~mm})$ to ensure surface wave suppression at both frequencies [26]. In addition, to ensure only the fundamental mode exists in the corrugation cavities, the thickness of the metallic walls, $t$, and the spacing between the walls, $s$, have to satisfy the following condition [52]:

$$
t+s<\lambda / 2
$$

The properties of the choke ring were confirmed by a model developed in [25], which predicts the far-field amplitude and phase pattern of the DM antenna on top of the choke ring. The model showed that the choke ring improves the radiation pattern by imposing a sharp gain roll-off at the horizon and reducing side and back lobes, owing to its surface wave suppression capabilities.

\subsection{Literature Review}

Multipath can be corrected at the receiver using digital signal processing (DSP) techniques, or rejected at the antenna. DSP solutions may involve real-time processing [53] or post-processing techniques [54], [55]. However, DSP only works for long delay multipath when the receiver can recognize the reflected signal as a delayed version of the direct LOS signal and discard it [26]. Short delay multipath signals are harder to recognize so they must be prevented from reaching the antenna, either by using a ground plane or a stand-alone antenna with good multipath rejection. The literature review will discuss antenna and ground plane solutions to multipath interference for various applications.

In [24], two low-profile ground plane solutions for multipath rejection at L1 were presented. The first solution is a dielectric-filled metallic cup ground plane, which is similar to the choke ring except that it has one outer ring only. The dielectric chosen was Rogers 3010 with $\epsilon_{r}=10.2$, which allowed the height to be reduced from $\lambda / 4=47 \mathrm{~mm}$ to $\lambda_{g} / 4=19.4 \mathrm{~mm}$. The second solution is a low-profile choke ring for L1 operation which was designed using the same technique of dielectric fillings to

reduce the mass and volume. It was shown that the metallic cup and the choke ring have similar radiation performances at L1. The drawback of these designs is they cannot be used for dual- and wide-band applications because the dielectric imposes a narrow AMC bandwidth (this will be demonstrated in Sec. 2.4.1).

The invention of the mushroom high impedance surface [34] has inspired a number 
of ground plane designs for multipath mitigation. In [14], [28], a broadband PCBbased AMC ground plane was designed to operate in the GNSS band. This AMC design is a periodic array of two via-grounded copper patches with Rogers 4350 between them. Nomex honeycomb material is used as a spacer between the periodic structure and the ground plane. The design has $\pm 90^{\circ}$ reflection phase response and a surface wave bandgap across the entire GNSS band. The radiation characteristics were tested with the DM antenna on the AMC structure and compared with the choke ring. Results showed the AMC structure had acceptable multipath performance but it was inferior to the conventional choke ring at both L2 and L1.

Another paper explored the electromagnetic band gap property of the mushroom structure to design an EBG surface for multipath suppression. The structure exhibits surface wave suppression within the GNSS band [56]. The design was tested with the DM antenna and compared with the choke ring and was found to have comparable radiation characteristics with the advantage of being lighter and shallower than the choke ring. The disadvantage of this design is having a large diameter similar the choke ring.

In [57], an AMC structure designed to have zero reflection at L1 was tested with a microstrip antenna. It was shown that the AMC ground plane improves RHCP gain at zenith and reduces the LHCP gain, especially below horizon, hence improving the down-up ratio. The drawback of this design is that it is single-band.

A novel corrugated surface was presented in [26], [58]. The new design has corrugation depths $d \leq \lambda / 4$ and achieves multipath rejection by destructive interference of surface waves, as opposed to the choke ring which has corrugation depths $\lambda / 4<d<\lambda / 2$ and achieves multipath rejection by surface wave suppression. Due to its depth, the new design operates in the surface-wave non-cutoff in which "slow" surface waves can propagate. The idea is to have the LOS signal and the surface wave cancel at the edge of the corrugated surface, therefore eliminating edge diffraction. Wave cancellation is achieved by the design of the corrugation depth, ring gap, tooth thickness, number of rings, and height of radiating element above the corrugated surface. The non-cutoff design has a lower profile than the choke ring and has similar radiation performance. The drawback of this design is greater cost and fabrication complexity due to the large number of corrugations.

A novel dual-band choke ring for L1 and L2 operation was designed by incorporating an electromagnetic filter in the choke ring grooves [59], [60]. The presence of the filter makes the depth of the groove appear different depending on the frequency of the signal. The filter is designed to reflect L1 signals and allow L2 signals to pass through. The filter is placed at a depth equal to the quarter-wavelength at L1 such that L1 signals see that depth as the depth of the grooves. On the other hand, L2 signals pass through the filter and see the full depth of the groove. The dual-band choke ring was tested and has shown superior performance than the conventional choke ring in terms of down-up ratio both L1 and L2. The drawback of this design is 
its fabrication complexity.

A novel multi-band (L1, L2, \& L5) antenna supplemented with a novel choke ring design was presented in [61], [62]. The novel choke ring design is made from metallic disks with cylinders of varying diameters placed between the disks. The novel choke ring has a lower profile than the conventional choke ring, but their performances have not been compared.

A planar dual-band (L1 \& L2) microstrip antenna based on the reduced surface wave (RSW) concept was developed in [63] (an L1 only version of the same antenna was introduced in [64]). The RSW concept was developed by Jackson et. al [65]. An RSW antenna is characterized by having low susceptibility to multipath interference without the need for an additional ground plane for surface wave suppression. To achieve this goal, the antenna has low horizon and back radiation combined with high cross-polarization rejection above horizon. The antenna was tested and compared with the choke ring and was found to have better radiation performance, especially in rejecting low-angle multipath. Other antenna designs based on the RSW concept can be found in [66], [67], [68]. The drawback of these antennas is that they are narrow-band.

A novel antenna, called the Pinwheel antenna, with a stable phase center and good multipath rejection was presented in [69]. The design is a phased array of aperture-coupled spiral slots for radiation and an outside array of concentric slot rings for suppression of diffracted and reflected signals. The result is an antenna that receives low-elevation RHCP signals while rejecting low-elevation LHCP signals which normally arise from multipath. The drawback of this antenna design is its fabrication complexity.

A novel choke ring structure was designed by Leica which uses rings with different heights and slots within the rings [70]. The largest ring height is approximately equal to quarter-wavelength at L2 while the smallest height is approximately equal to quarter-wavelength at L1. The main advantage of the design is improving multipath mitigation while also tracking low elevation satellites. This is due to the design's conical shape which does not force a sharp gain roll-off near horizon, unlike the choke ring. However the design has the disadvantage of being bulkier, heavier, and more expensive to manufacture than the conventional choke ring.

With the same design goal of improving low-elevation gain as Leica, Topon designed a convex ground plane using straight pin on a convex shaped ground plane [71]. Similar to Leica's design, Topcon's design improves low-elevation gain while maintaining good multipath signal rejection as evident from the down-up ratios reported for L1 and L2.

Other techniques for multipath mitigation presented in the literature include a low-profile ceramic choke ring with surface tapering for moisture run-off and a novel cheaper manufacturing process [72], a single-feed wideband GNSS patch antenna on 
a conventional choke ring [73], a crossed-dipole wideband reference antenna for GPS and Galileo on a wideband AMC ground plane [22], a single-band (L1) Galileo patch antenna on an EBG substrate with acceptable multipath rejection and axial ratio performance but worse than the choke ring [21], a wide-band monopole antenna over a dual-band tunable AMC for reduced back radiation and improved boresight gain [74], a dual-band dipole antenna for WLAN applications on a dual-band AMC ground plane with increased front gain and reduced back radiation [75], a single-band (L1) microstrip patch antenna on a hexagonal EBG substrate [76], and an X-band (8.025$8.4 \mathrm{GHz}$ ) choke ring horn antenna for multipath mitiation for space applications [77], and a choke ring structure for surface wave suppression for aviation applications [78].

\subsection{Proposed Design}

\subsubsection{Choke Ring Analyses}

Choke ring theory was presented in Sec. 2.2.5. To further study the properties of the choke ring. Finite element method (FEM) High Frequency Structure Simulator (HFSS) was used to measure the reflection phase and extract the corresponding surface impedance characteristics as shown in Fig. 2.4. An incident plane wave was excited using a waveport at distance of $150 \mathrm{~mm}$ from surface of the choke ring and de-embedded to the surface to get the correct reflection phase and surface impedance [79], [80].

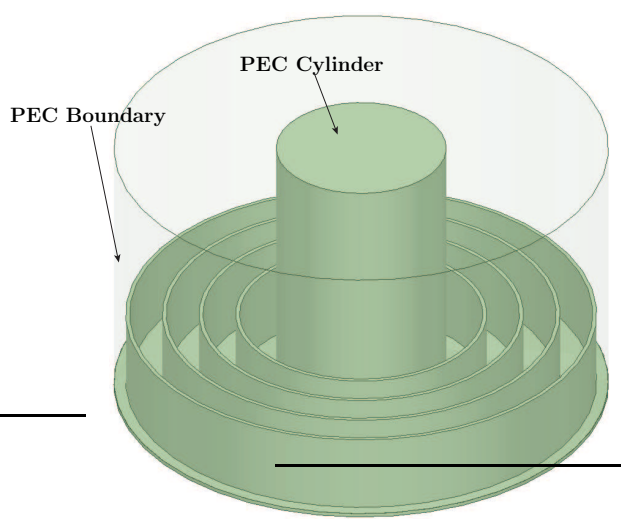

(a)

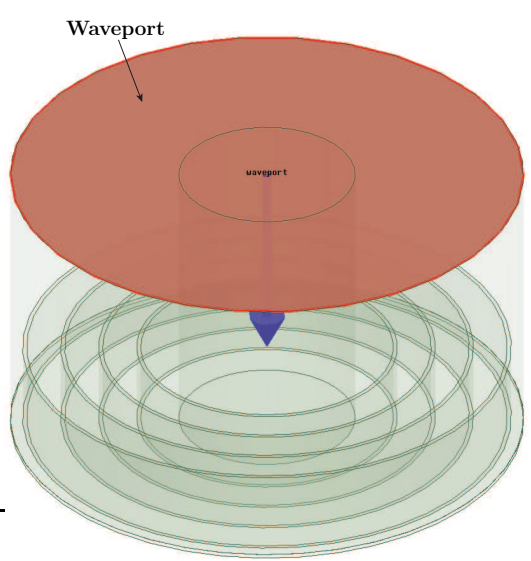

(b)

Figure 2.4: FEM-HFSS choke ring model used to compute reflection phase and surface impedance.

The zero reflection phase corresponds to the resonant frequency of the surface, beyond which the surface impedance becomes capacitive as shown in Fig. 2.5. For a conventional metallic choke ring with $d=61.3 \mathrm{~mm}$, the desired frequency L2 $(\lambda / 4=$ 
$61 \mathrm{~mm})$ is thus slightly to the right of this resonance to achieve a highly capacitive response required for efficient surface wave suppression, as shown in Fig. 2.5(b). On the other hand, it exhibits a moderate but still capacitive response at the second frequency band L1, thereby making the choke ring operation acceptable at this band.

To achieve a compact and a low-profile choke ring, a metallic air choke ring can be replaced by a dielectric-filled choke ring design, as introduced in [24]. However, this comes at the expense of narrower AMC bandwidth and reduced wave suppression capabilities, at the second band, L1. To demonstrate this, FR4 $\left(\epsilon_{r}=4.4\right.$, $\tan \delta=0.02)$ and Rogers $6010\left(\epsilon_{r}=10.2, \tan \delta=0.0023\right)$ dielectrics were used to fill the corrugation cavities and the corrugation depths were adjusted for the guided quarter-wavelength in each dielectric. Just like with the conventional choke ring, the corrugation depths chosen were slightly larger than $\lambda_{g} / 4$ to achieve capacitive surface impedance for TM surface wave suppression, at the design frequency L2. As shown in Fig. 2.5, the larger the dielectric constant, the narrower the AMC bandwidth (defined as the range of frequencies where reflection phase is in the range of $\pm 90^{\circ}$ ). Having a narrow AMC bandwidth around L2 makes dielectric choke rings inadequate for wideand dual-band GNSS applications because the reflection phase at L1 deviates significantly from zero and starts to appear as a metallic ground plane. As a consequence of the narrow AMC bandwidth, the capacitive surface impedance bandwidth also gets narrower as shown in Fig. 2.5(b) where the surface impedance approaches zero faster, as the dielectric constant becomes higher. As a result, the TM surface wave suppression degrades at the higher frequency band (L1).

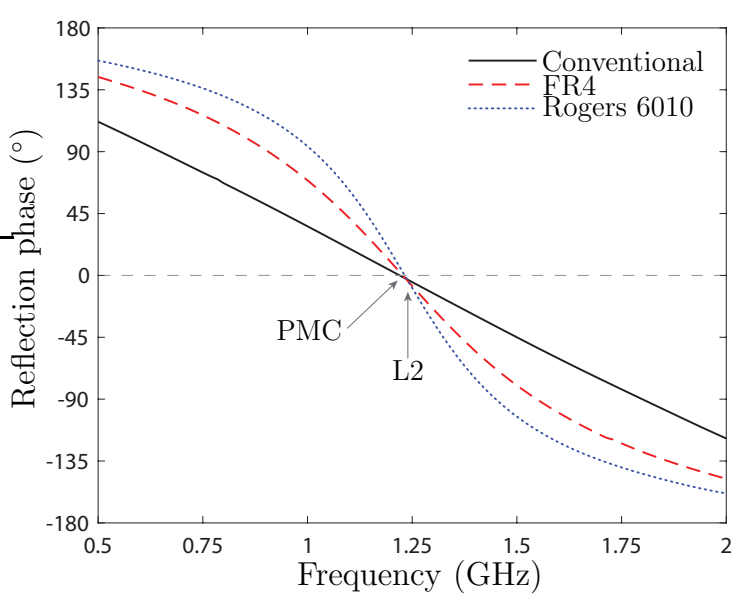

(a)

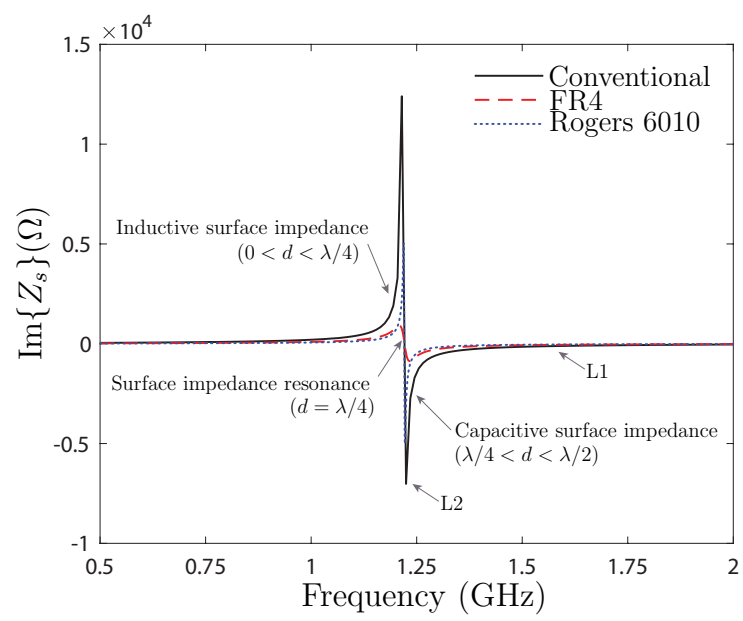

(b)

Figure 2.5: Comparison of the EM properties of three choke ring configurations: a conventional choke ring, a choke ring with corrugation cavities filled with FR4, and a choke ring with corrugations cavities filled with Rogers 6010. a) Reflection phase, and b) surface impedance. 


\subsubsection{Proposed Dielectric Choke Ring}

To solve the problem of narrow bandwidth of dielectric based choke rings, a new structure is proposed here, where a second resonance is created in the surface impedance of the choke ring, around the L1 band, in addition to the one at L2. This effect can be achieved by using two different dielectric fillings inside the choke ring at optimal locations, as shown in Fig. 2.6(a). By varying the dielectric constants and their locations, the dispersion, and thus the surface impedances, can be engineered at both L2 and L1 bands. This concept is similar to the choke rings using multiple corrugation depths to achieve a multi-band response, at the cost of high complexity and costly fabrication [70].

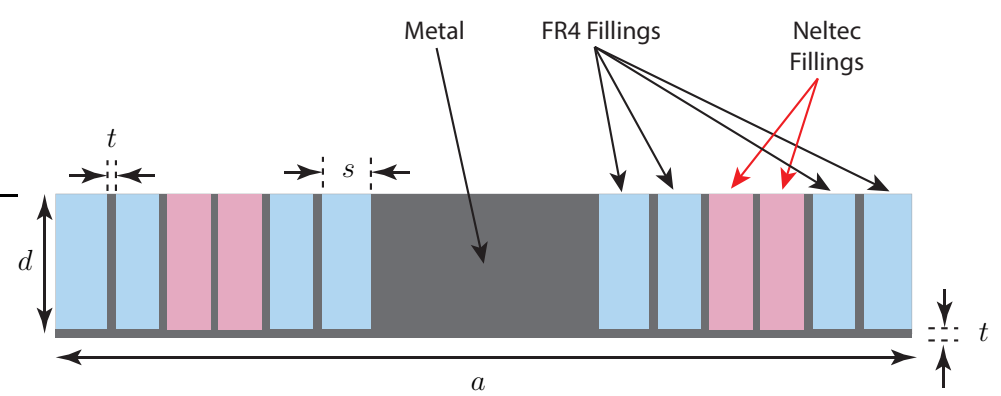

(a)

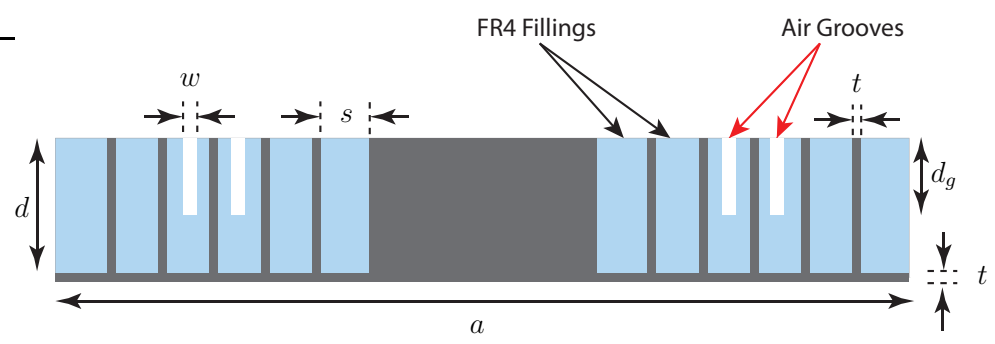

(b)

Figure 2.6: Two electromagnetically equivalent dielectric choke rings. a) Multidielectric choke ring made by filling corrugation cavities with FR4 and Neltec NX9240. The dimensions of the structure required to achieve dual resonance at L2 and L1 are as follows: $a=280 \mathrm{~mm}, s=10 \mathrm{~mm}, d=29.2 \mathrm{~mm}$, and $t=3 \mathrm{~mm}$. b) Proposed grooved dielectric choke ring made by filling corrugation cavities with FR4 with air grooves. The dimensions of the structure required to achieve dual resonance at L2 and L1 are as follows: $a=280 \mathrm{~mm}, s=10 \mathrm{~mm}, d=29.2 \mathrm{~mm}, d_{g}=15 \mathrm{~mm}, w=$ $4 \mathrm{~mm}$, and $t=3 \mathrm{~mm}$.

To demonstrate the dual-band nature of the proposed structure, Fig. 2.7 shows the reflection phase and the surface impedance achieved using a multi-dielectric design of Fig. 2.6(a), with four cavities filled with FR4 and two filled with Neltec NX9240 $\left(\epsilon_{r}=2.4, \tan \delta=0.0016\right)$. As can be seen, the structure exhibits zero reflection phase, corresponding to two resonant frequencies, around the L2 and L1 bands. More importantly, by engineering the geometrical dimensions of the structure, the surface 
impedance is now made highly capacitive at L1, as required for efficient surface wave suppression at both the bands.

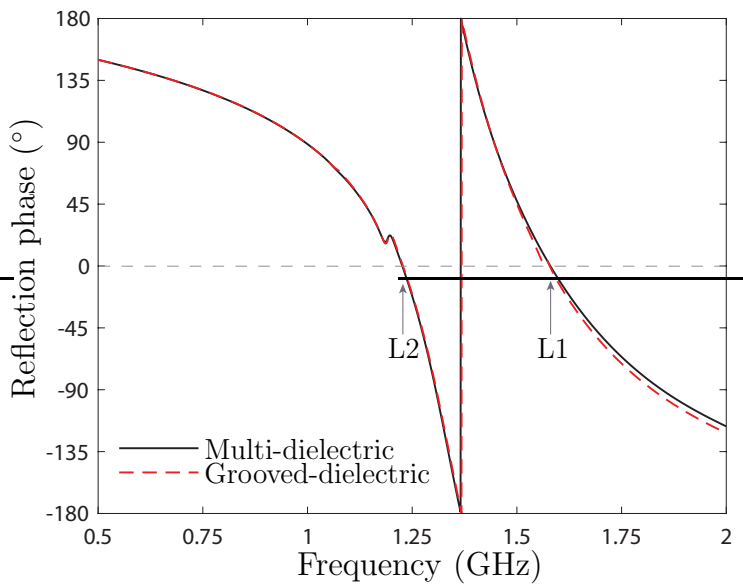

(a)

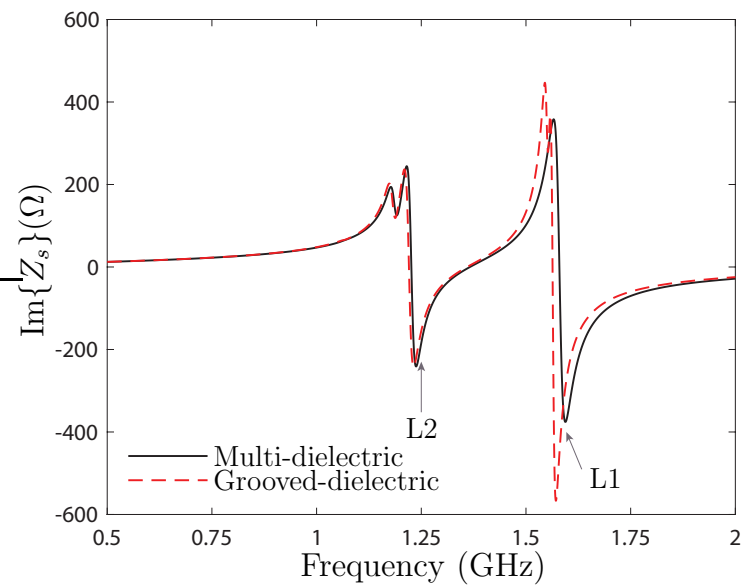

(b)

Figure 2.7: Dual-band EM response of the multi-dielectric design and the proposed grooved dielectric design. a) Reflection phase, and b) surface impedance.

The same dual-band response can also be achieved by replacing Neltec NX9240 with FR4 with air-grooves. The grooved-dielectric design, shown in Fig. 2.6(b), is a choke ring with all cavities filled with FR4 and with grooves made within the two FR4 fillings where there was Neltec NX9240. Compared to the multi-dielectric choke ring, the grooved dielectric choke ring is advantageous from a fabrication and cost point of view as it uses only one dielectric material. Moreover, the grooved-dielectric design provides extra degrees of freedom to control the dual resonance response using one dielectric filling material, so as to achieve a high capacitive surface impedance at two independent frequencies. This is demonstrated in Fig. 2.7 where the grooved-dielectric design shows nearly identical reflection phase and surface impedance compared to the multi-dielectric design.

Next, Fig. 2.8 shows the comparison between the surface impedances of the full FR4 choke ring (i.e. ungrooved) and the grooved FR4 choke ring. While introducing the grooves adds a second resonance around L1 with the desired capacitive response, it also results in a lower capacitive surface impedance at the lower band L2, compared to the original design. In this sense, the proposed structure has a trade-off where it improves the performance at L1 at the expense of a degradation at L2. While this may appear as a drawback of the proposed choke ring, it will be shown in the the full-wave (Sec. 2.4.3) and experimental (Sec. 2.4.4) demonstrations that the surface impedance achieved in both bands is sufficiently capacitive to provide excellent surface wave suppression in both bands simultaneously.

Finally, to further understand the behavior of the grooved choke ring structure, a parametric study was done. It was found that the depth $\left(d_{g}\right)$ of the FR4 grooves allows for tuning of the second AMC frequency point (L1), as shown in Fig. 2.9(a). 


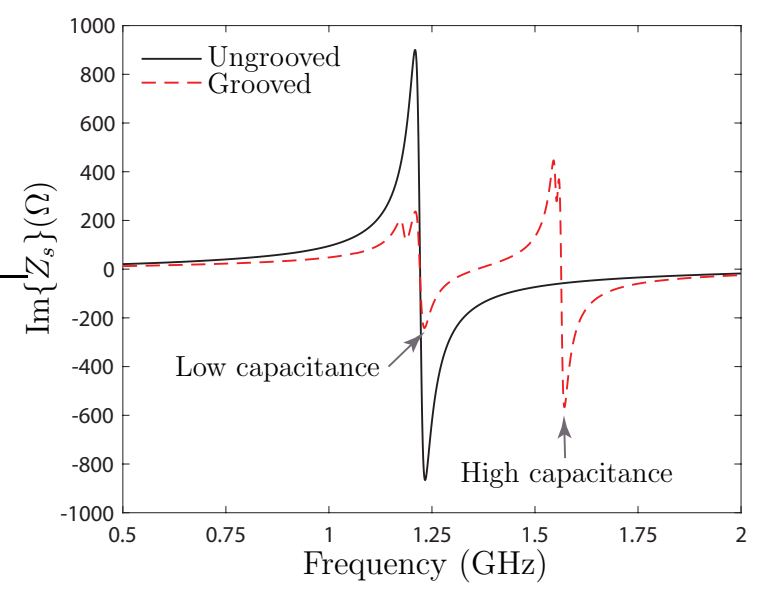

Figure 2.8: Surface impedance comparison between ungrooved and proposed grooved FR4 choke rings.

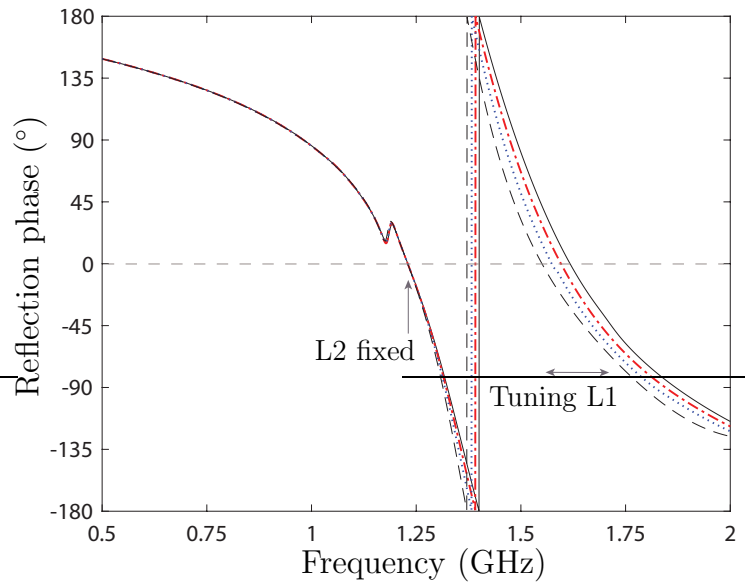

(a)

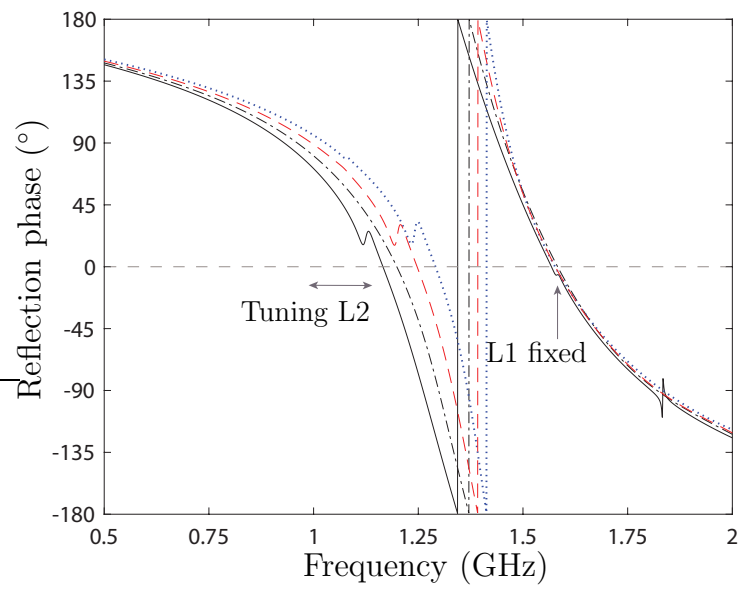

(b)

Figure 2.9: Parametric study of the grooved-dielectric design to independently control two AMC points for dual-band surface suppression operation. a) Tuning the second frequency L1 by changing the depth of the FR4 grooves, and b) tuning the first frequency L2 by changing the height of the whole structure and the depth of the FR4 grooves.

In order to tune the L2 frequency, the height $(d)$ of the whole structure and the depth of the FR4 grooves $\left(d_{g}\right)$ must be changed simultaneously, as shown in Fig. 2.9(b). In both cases, it is seen that it is possible to independently shift the two AMC points (and the surface impedance resonances) by optimizing the height and the depth of the structure. 


\subsubsection{Full-Wave Demonstration}

The Dorne \& Margolin (DM) antenna, shown in Fig. 2.10(a), was used to investigate the multipath rejection performance of the conventional choke ring and the proposed dielectric choke ring. The DM antenna is a widely used antenna for GNSS geodetic applications [14] [56]. It is a compact RHCP antenna based on a half Alford Loop [81] with a wideband response covering the entire GNSS band. First, the DM antenna was simulated by itself. Next, the DM antenna was simulated on top of the conventional choke ring and the proposed dielectric choke ring as shown in Fig. 2.10(b), Fig. 2.10(c), respectively. The simulated results at L2 and L1 are shown in Fig. 2.11.

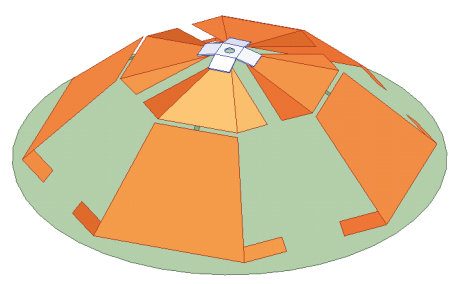

(a)

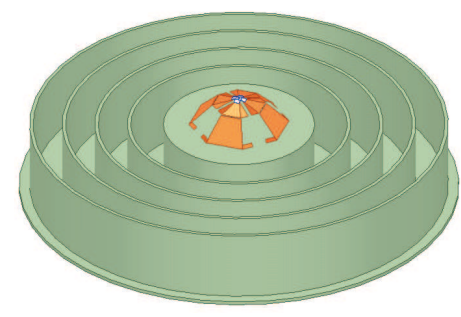

(b)

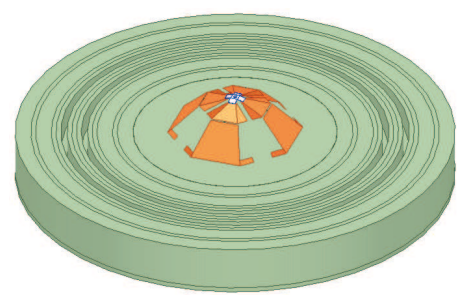

(c)

Figure 2.10: FEM-HFSS simulation models of the Dorne \& Margolin (DM) antenna in various configurations: a) stand-alone, b) on top of a conventional choke ring, and c) on top of the proposed dielectric choke ring.

FEM-HFSS results show that at L2 the proposed design has slightly less RHCP gain at zenith $\left(\theta=0^{\circ}\right)$ compared to the choke ring, and a higher LHCP gain in the back radiation (outside $\pm 90^{\circ}$ ). On the other hand, the proposed design performs better than the conventional choke ring at L1, exhibiting higher RHCP gain at zenith and lower LHCP gain at horizon $\left(\theta= \pm 90^{\circ}\right)$. The desired characteristic of using the choke ring and proposed design is the sharp RHCP gain rolloff from zenith to horizon and low back radiation, compared to the stand-alone DM antenna, which is evident from the radiation plots in Fig. 2.11. For example at L2, the RHCP gain rolloff of the stand-alone DM from zenith $(4 \mathrm{~dB})$ to horizon $(-4 \mathrm{~dB})$ is only about $8 \mathrm{~dB}$, while for the proposed design it is nearly $19 \mathrm{~dB}$.

The corresponding down-up ratios at L2 and L1 shows the multipath performance in terms of rejection of reflections coming from the ground beneath the antenna. While the proposed design performs worse than the choke ring at L2, as expected from Fig. 2.8, it still has good performance with a down-up ratio lower than $-25 \mathrm{~dB}$ at zenith. The dual-band structure is better than the choke ring at L1, especially near the horizon. This degradation of performance at L2, while maintaining excellent radiation characteristics at L1, is the primary trade-off in using the proposed design. The axial ratios at L2 and L1 are also shown in Fig. 2.11. The choke ring and the proposed design have comparable axial ratio in the range of $\pm 45^{\circ}$ at L2 and L1. Outside the $\pm 45^{\circ}$ range, at L2, the dual-band structure has a higher axial ratio with the worst case at horizon, at which point it is higher than the choke ring by $1 \mathrm{~dB}$. 

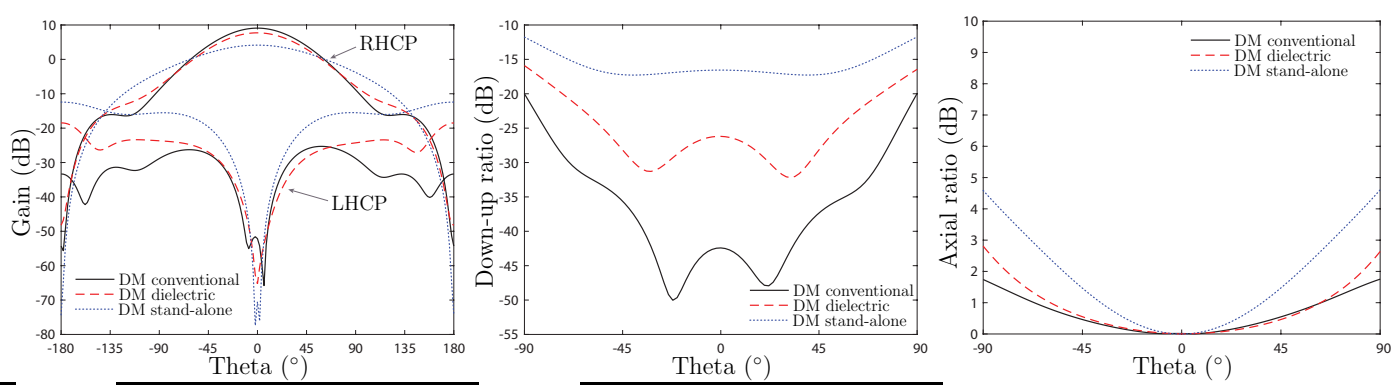

(a)
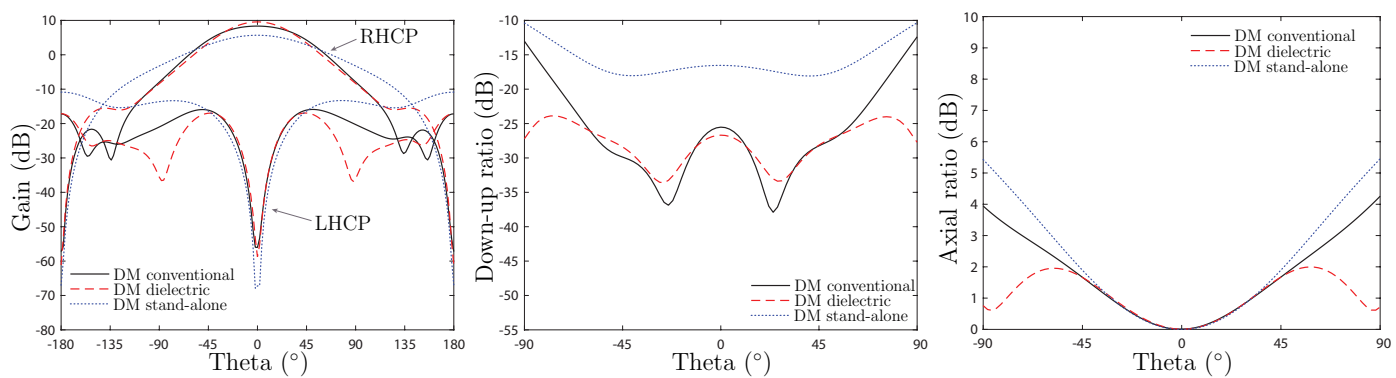

(b)

Figure 2.11: FEM-HFSS simulated radiation patterns, down-up ratio, and axial ratio of the DM antenna in various configurations: standalone, on top of a conventional choke ring, and on top of the proposed dielectric choke ring at a) L2 and b) L1 frequency bands. All plots are at $\phi=0^{\circ}$ principal cut.

At L1, the dual-band structure has a lower axial ratio, with the best case scenario occurring at horizon. Finally, it is seen that the choke ring and the proposed design have superior multipath rejection performance compared to the stand-alone DM at both L2 and L1, owing to their surface wave suppression characteristics. 


\subsubsection{Experimental Demonstration}

\subsubsection{Design Prototype}

It was decided to fabricate a prototype of the proposed design using a single machined FR4 part, a plurality of copper nails, and circular copper tape rings as shown in the simulation model in Fig. 2.12. Borrowing from the concept of substrate integrated waveguides (SIW) where a plurality of conducting posts or vias are used to replace metallic walls [82], the walls of the choke ring can be replaced by copper posts. In SIW, the propagation characteristics can be made similar to rectangular waveguides by making the metallic posts closely packed. The same concept can be applied to the choke ring walls. The characteristics of a choke ring with posts will approach that of a choke ring with walls the more closely packed the posts are.
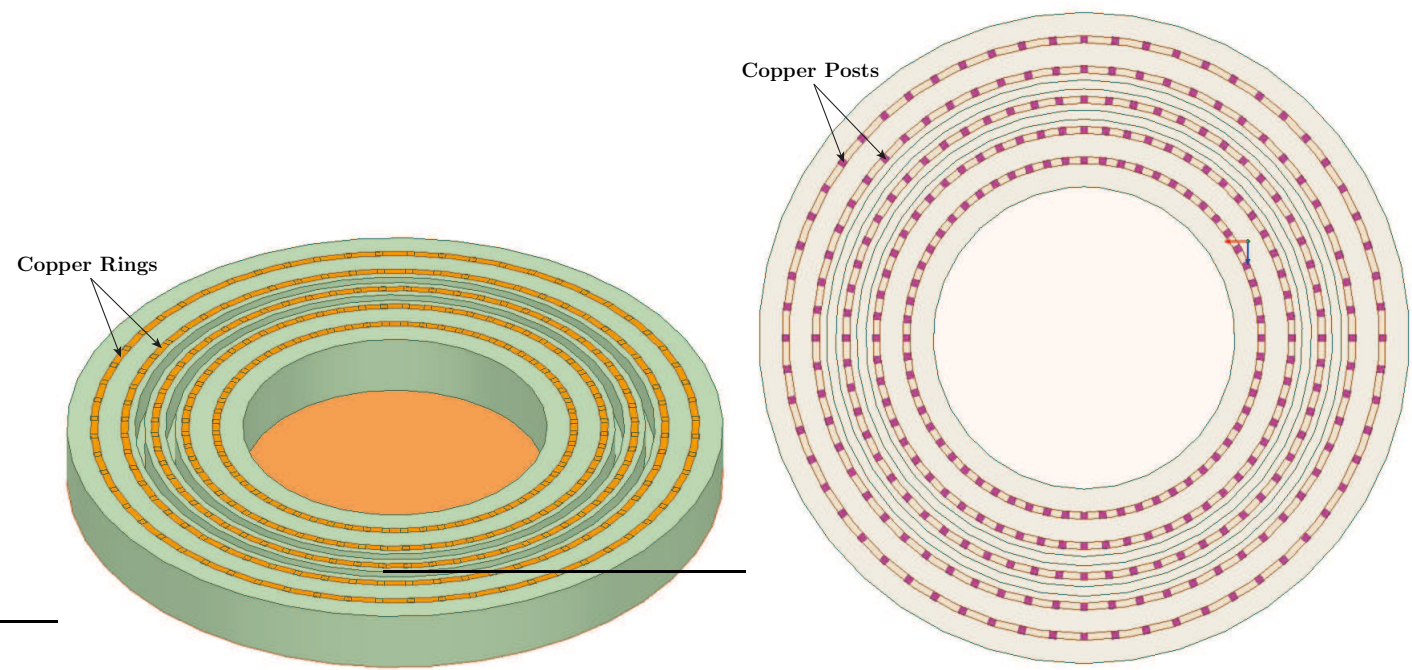

Figure 2.12: FEM-HFSS model of the proposed dielectric choke ring implementation with copper posts replacing walls.

First, the effect of replacing the metallic walls with metallic posts was simulated. Fig. 2.13(a) shows the reflection phase response for the proposed design with metallic walls and various cases involving different numbers of metallic posts. It is seen that replacing the walls with posts detunes the reflection phase response. The reflection phase of the structure with posts approaches that of the metallic walls the greater the number of posts, as expected. While using the greatest number of posts possible is advantageous from a performance point of view, it would result in higher costs and fabrication complexity. In an effort to find the best compromise between cost and performance, it was decided to replace with metallic walls with a total of 300 posts (Case 2 in Fig. 2.13(a)).

It was then discovered that adjusting the height of FR4 and the depth of the FR4 corrugations fixes the reflection phase detuning problem. Fig. 2.13(b) shows that 


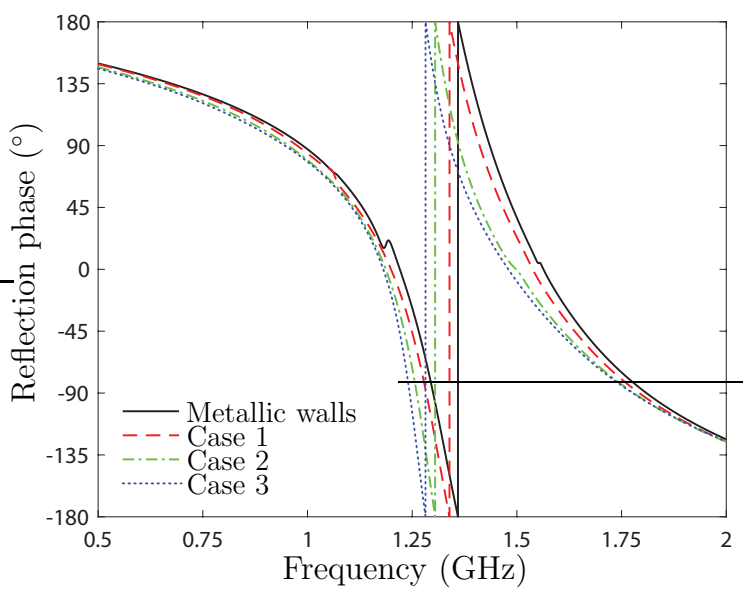

(a)

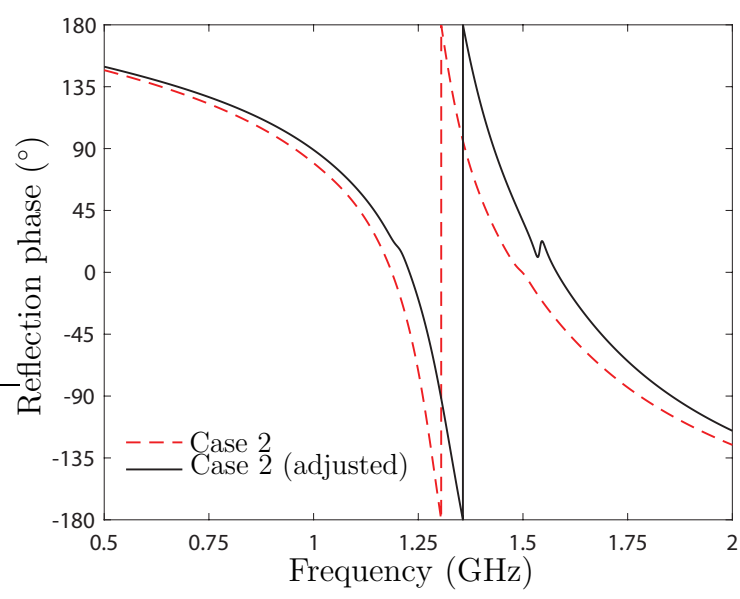

(b)

Figure 2.13: a) Reflection phase of multiple number of copper posts replacing metallic walls. Number of posts replacing each ring, starting from the inner wall: Case 1 (90, $120,120,180,180[$ total $=600])$, Case $2(60,60,60,60,60[$ total $=300])$, and Case 3 $(36,40,45,60,60$ [total $=241])$, and b) Case 2 before and after adjusting dimensions to get the phase response close to metallic walls.

the reflection phase response of the design with 300 posts can be tuned as close as possible to the design with metallic walls, hence causing minimal distortion to the performance of the prototype. Additional dimension changes were dictated by the FR4 used in fabrication which has dielectric constant $\epsilon_{r}=4.8$ unlike $\epsilon_{r}=4.4$ used in the simulation. The final dimensions are shown in the drawing in Appendix A.

Fig. 2.14 shows the in-house prototype of the proposed grooved-dielectric choke ring design fabricated using a single machined FR4 part, a plurality of copper nails, and circular copper tape rings to connect the top and bottom of the copper nails. 

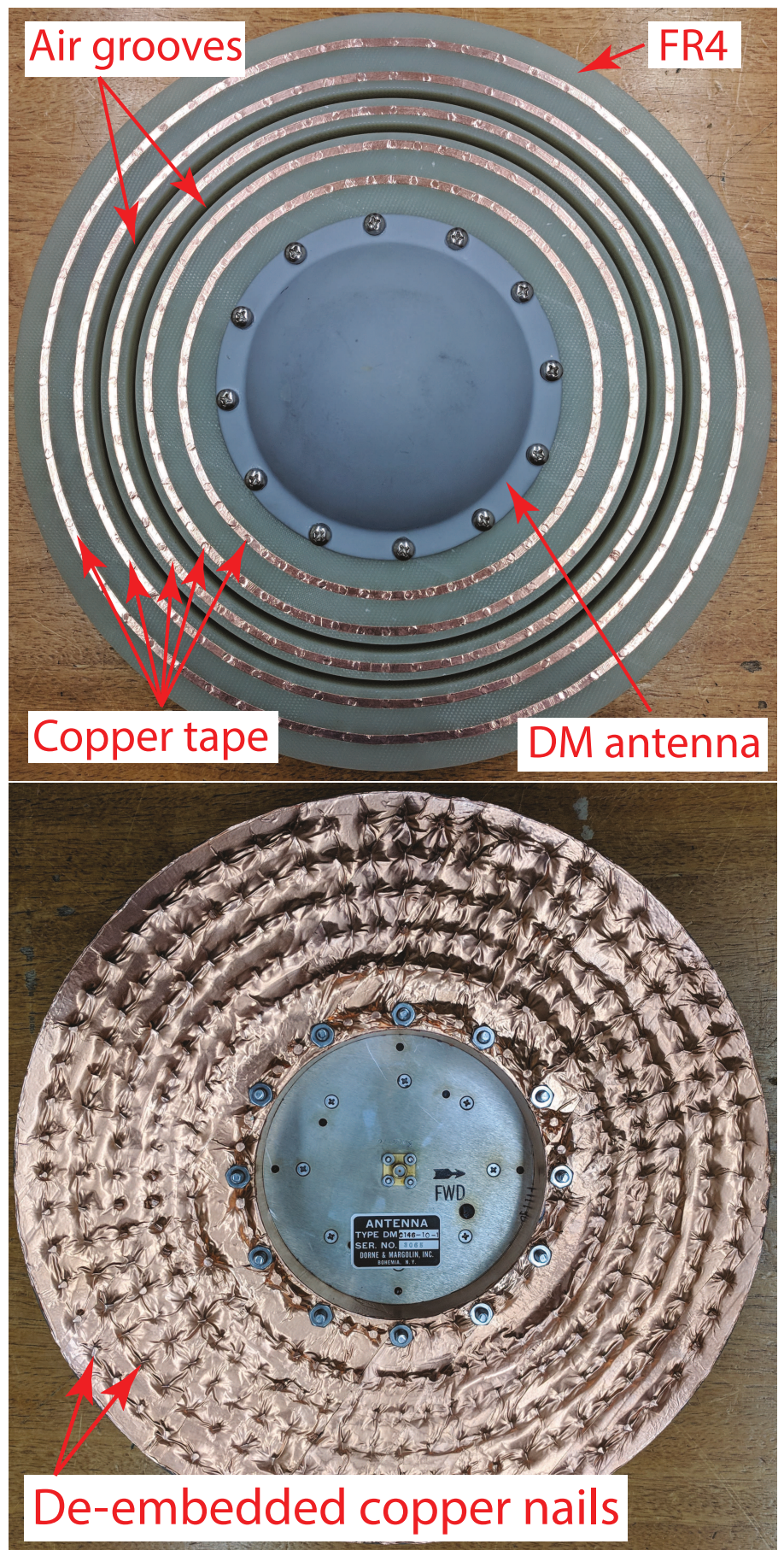

Figure 2.14: Top and bottom views of the fabricated grooved dielectric choke ring with the DM antenna. 


\subsubsection{Testing}

The DM antenna, stand-alone and on top of the conventional choke ring, was tested using the Satimo near-field system (frequency range: $800 \mathrm{MHz}$ to $6 \mathrm{GHz}$ ) at Polytechnique Montreal [83], as shown in Fig. 2.15(a) and Fig. 2.15(b). The device-under-test (DUT) is mounted on a turntable at the center of the chamber. The Satimo system uses fifteen pairs of two orthogonal dipoles set at 22.5 apart as shown in Fig. 2.15(a). To measure the 3D near field, the dipoles are electronically scanned and the turntable rotates the antenna. The near-field data is then post-processed to obtained the farfield. The RHCP and LHCP radiation patterns obtained from the Satimo system show the absolute gain of the antenna.

The DM antenna on top of the proposed design was tested in the far-field anechoic chamber at Carleton University, as shown in Fig. 2.15(c). The vector network analyzer (VNA) used in the test is a Keysight 8720ES VNA (50 MHz to $20 \mathrm{GHz}$ ). The VNA calibration was done using a standard short, open, load, and thru (SOLT) calibration kit. The transmit (Tx) antenna, connected to Port 1 of the VNA, is a Tallysman VP6000 passive GNSS antenna [84] which is configurable for RHCP or LHCP radiation. The receive $(\mathrm{Rx})$ antenna, connected to Port 2 of the VNA and mounted on a turntable, is the DM antenna on top of the proposed dielectric design. The transmission scattering parameter $\left(S_{21}\right)$ was obtained from the VNA for all elevation angles, $\theta$, and at the $\phi=0$ pattern cut. To get the absolute gain, the data was corrected by calibration using the DM antenna by itself, which has a known gain measured by the Satimo system. 


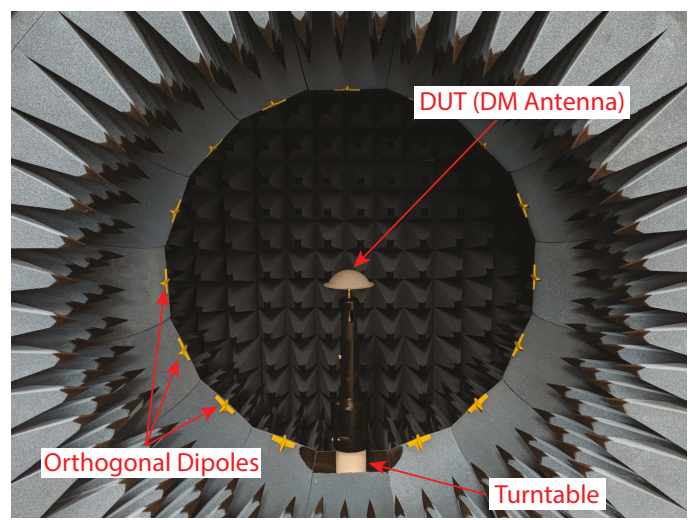

(a)

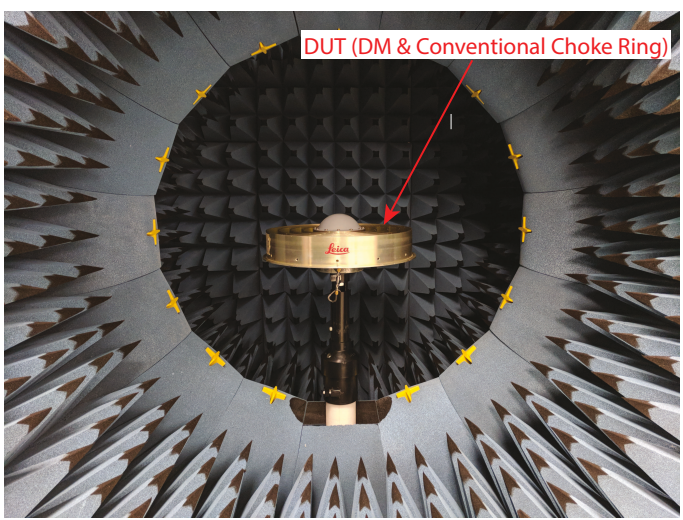

(b)

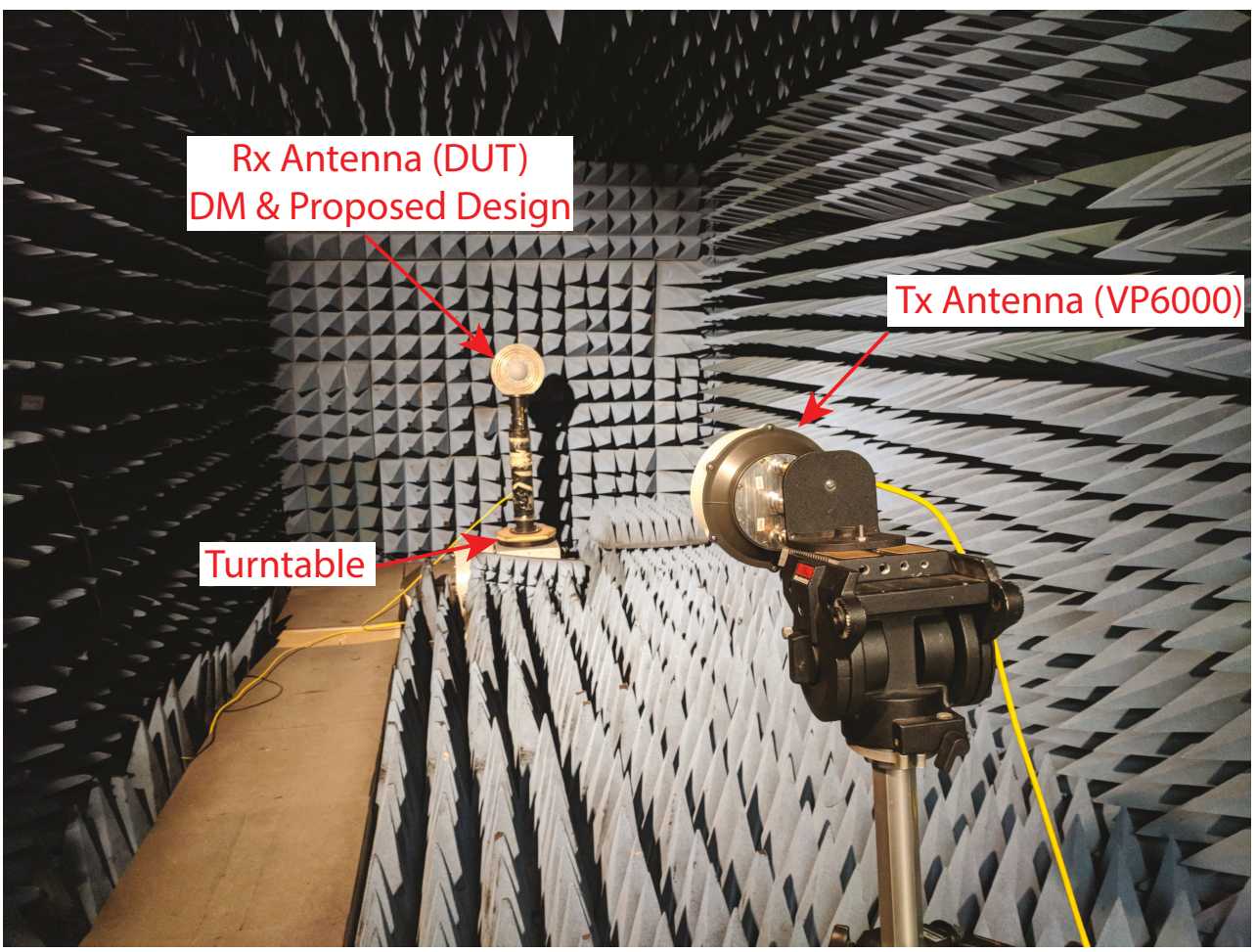

(c)

Figure 2.15: Testing the DM antenna in various configurations: a) stand-alone and b) on top of the conventional choke ring, using the Satimo system at Polytechnique Montreal, and c) on top of the proposed dielectric design in the anechoic chamber at Carleton University. 


\subsubsection{Results}

Fig. 2.16 shows the FEM-HFSS simulation and the measured radiation patterns of the DM antenna (stand-alone) at L2 and L1. While the DM antenna ideally exhibits zero cross-polarization as seen from the simulation results, a practical design features a finite LHCP gain, i.e. around $-20 \mathrm{~dB}$ at zenith. Moreover, it is also seen from Fig. 2.16 that the DM by itself lacks the sharp gain roll-off near horizon required for multipath rejection, which was expected from the simulations. For example, from Fig. 2.16(b) at L1, the RHCP gain roll-off from zenith $(5 \mathrm{~dB})$ to horizon $(-4 \mathrm{~dB})$ is only about $9 \mathrm{~dB}$.

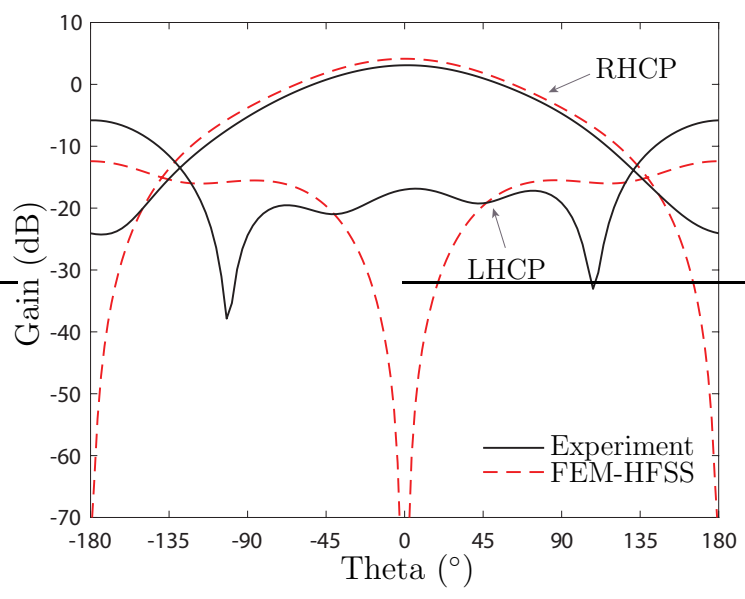

(a)

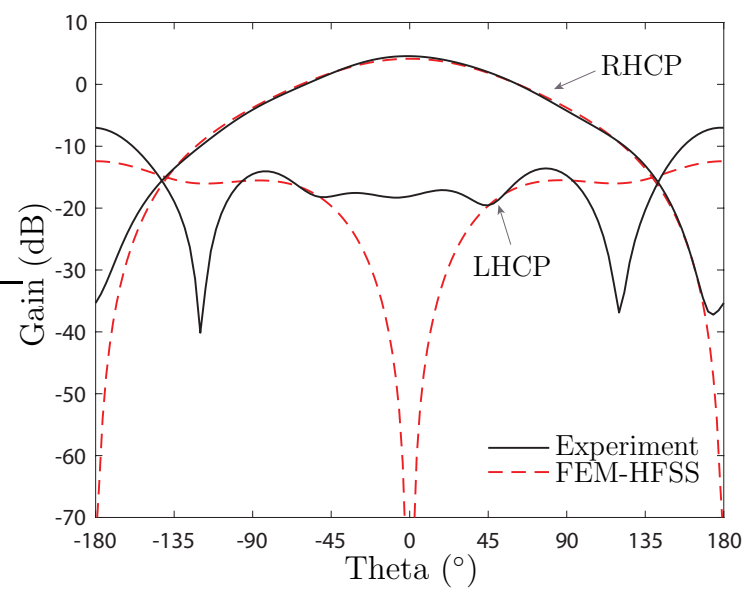

(b)

Figure 2.16: Experiment and FEM-HFSS simulation radiation patterns of the DM antenna at a) L2, and b) L1.

Fig. 2.17 and Fig. 2.18 show the measured radiation patterns of the DM antenna on top of the proposed dielectric choke ring at the desired L2 and L1 bands, along with several neighboring frequencies to account for any tolerances in fabrication. A good agreement is observed between the measurements and FEM-HFSS simulations. In particular, the radiation patterns of the proposed choke ring show a sharp gain roll-off near horizon for both RHCP and LHCP and low side and back lobes compared to the DM antenna by itself. For example, from Fig. 2.18(b) at L1, the RHCP gain roll-off from zenith $(9 \mathrm{~dB})$ to horizon $(-10 \mathrm{~dB})$ is about $19 \mathrm{~dB}$, which is $10 \mathrm{~dB}$ better than the DM without a ground plane. However, a high cross-polarization is observed in measurements, similar to that in the DM antenna by itself.

Fig. 2.19 and Fig. 2.20 show the measured DU ratios of the proposed dielectric choke ring and compared with that of the conventional metallic choke ring, at various frequency bands including L1 and L2. As expected from Fig. 2.7, high DU ratios are observed around the higher L1 band comparable to the conventional choke ring, due to higher capacitive surface impedances. On the other hand, the DU ratios in the lower L2 band, although not as high as the conventional choke ring, are still practically 


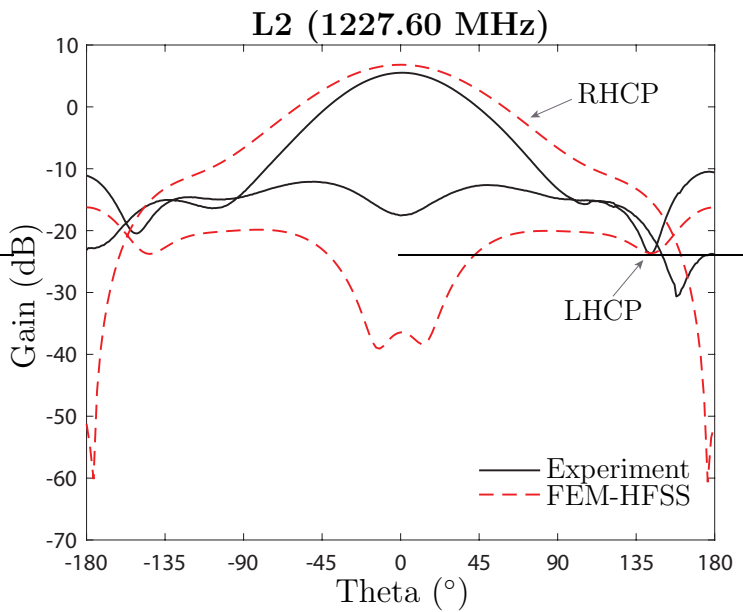

(a)

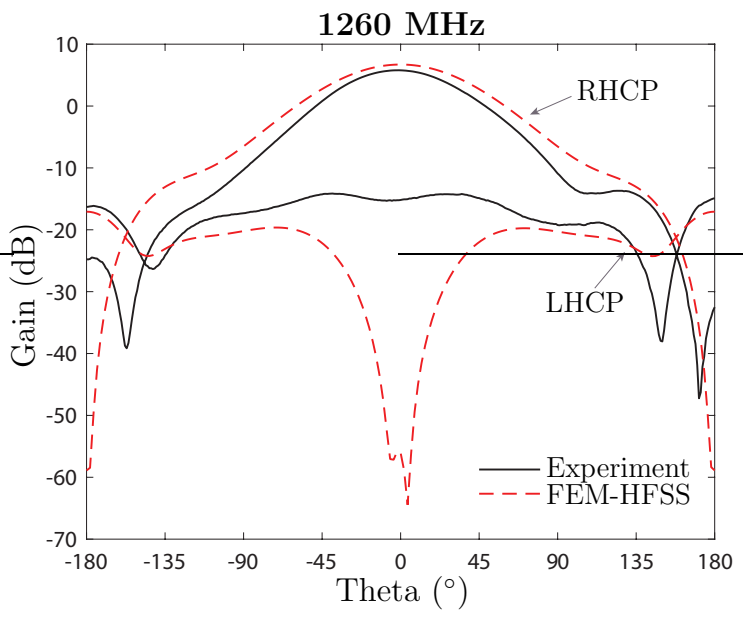

(c)

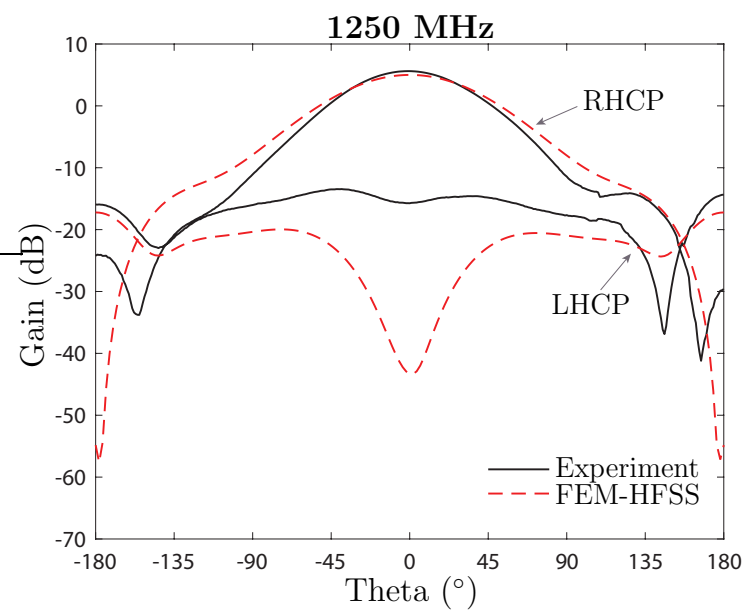

(b)

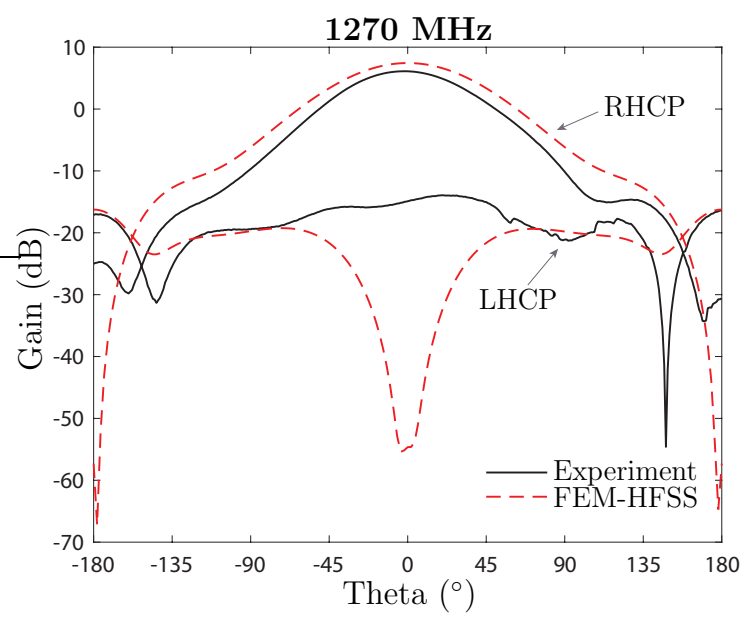

(d)

Figure 2.17: Comparison between the experiment and FEM-HFSS simulation results of the radiation patterns of the proposed design at L2 and various other frequencies around the L2 band a) L2 (1227.60 MHz), b) $1250 \mathrm{MHz}$, c) $1260 \mathrm{MHz}$, and d) 1270 $\mathrm{MHz}$.

high and comparable to that of the L1 band. In other words, the proposed choke ring successfully features high DU ratios, and thus strong surface wave suppression, around both L1 and L2 bands, while maintaining a compact and low-profile structure. 


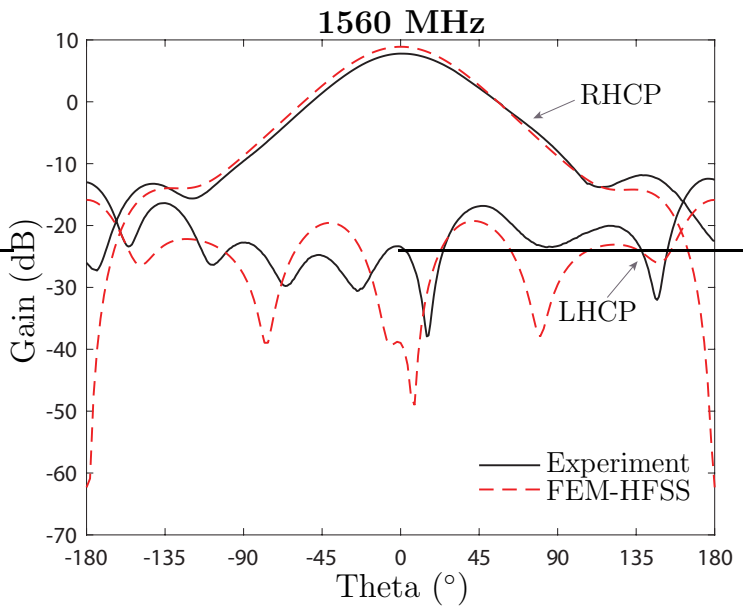

(a)

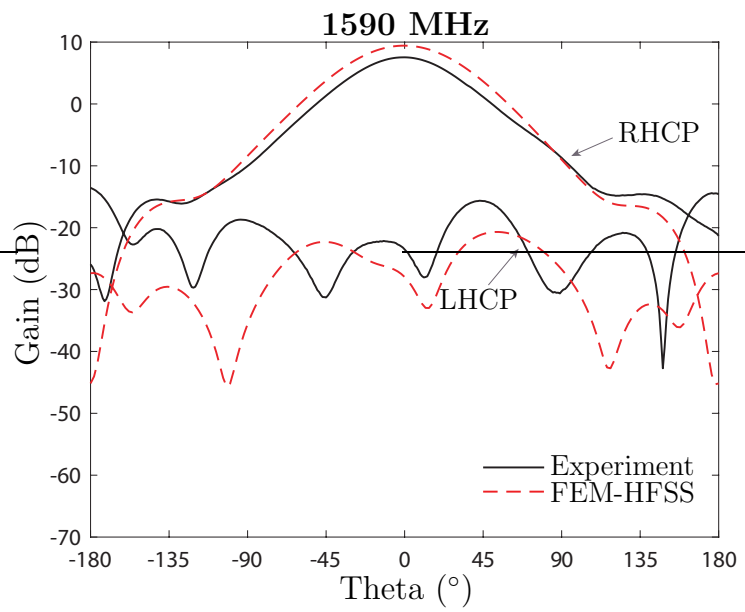

(c)

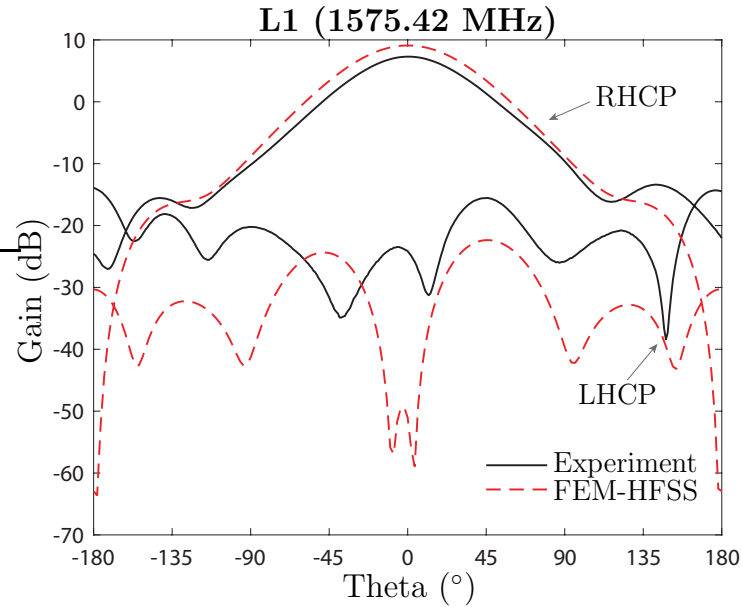

(b)

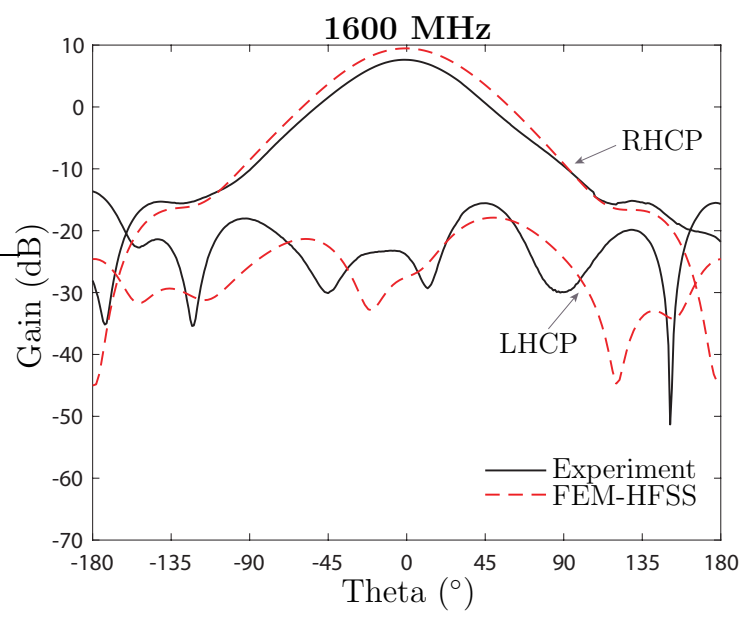

(d)

Figure 2.18: Comparison between the experiment and FEM-HFSS simulation results of the radiation patterns of the proposed design at L1 and various other frequencies around the L1 band a) $1560 \mathrm{MHz}$, b) L1 (1575.42 MHz), c) $1590 \mathrm{MHz}$, and d) 1600 $\mathrm{MHz}$. 


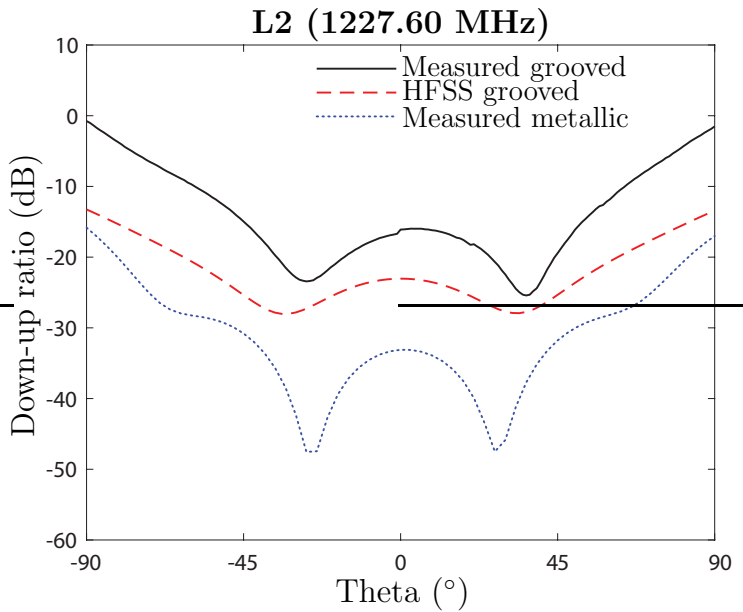

(a)

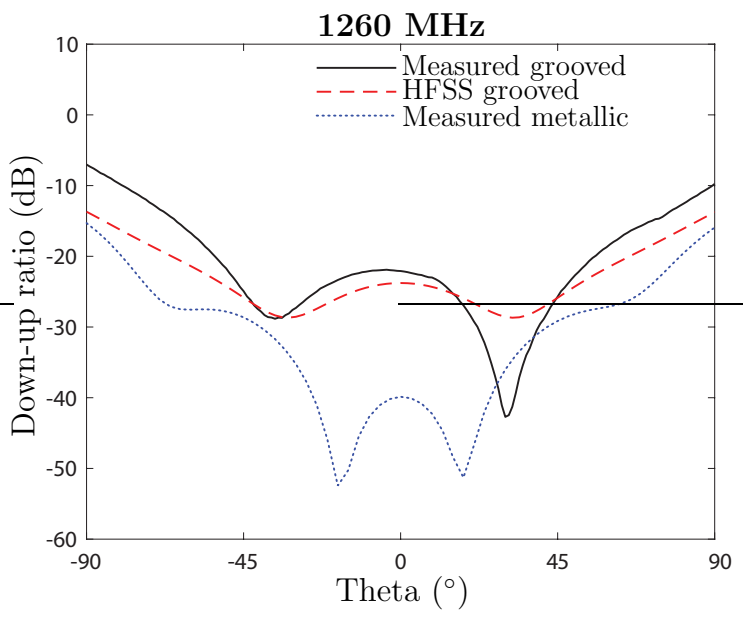

(c)

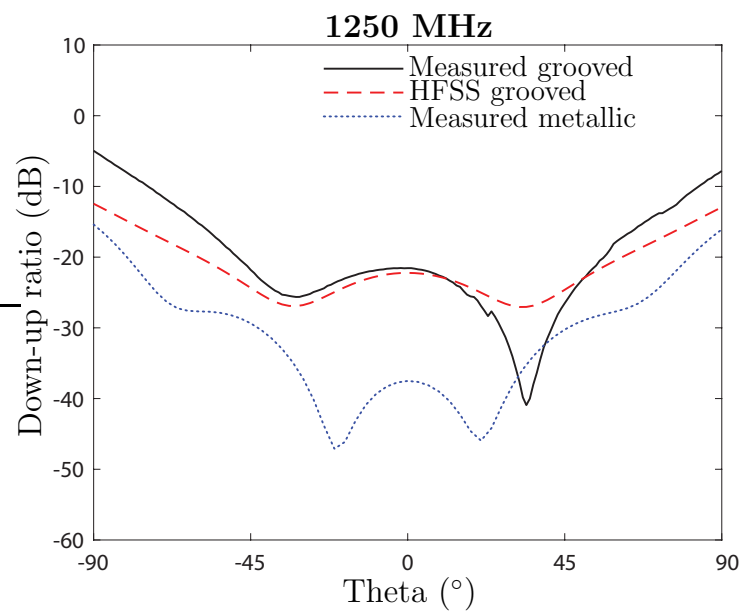

(b)

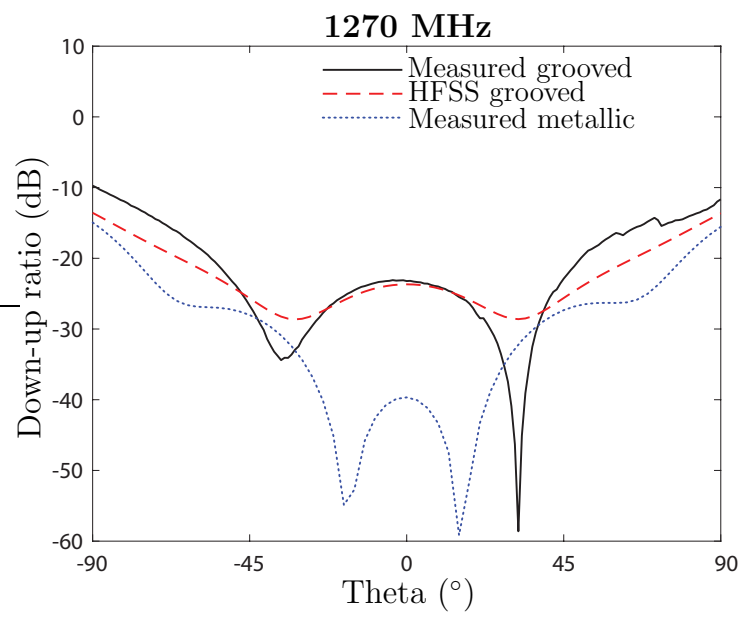

(d)

Figure 2.19: Comparison between the down-up ratio of the proposed design (experiment \& FEM-HFSS simulation) and the conventional metallic choke ring (experiment) at L2 and various other frequencies around the L2 band a) L2 (1227.60 MHz), b) 1250 $\mathrm{MHz}$, c) $1260 \mathrm{MHz}$, and d) $1270 \mathrm{MHz}$. 


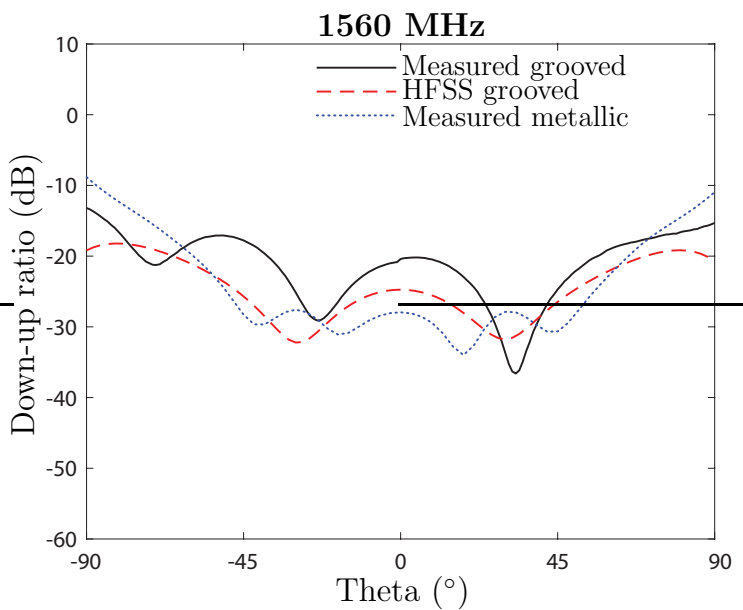

(a)

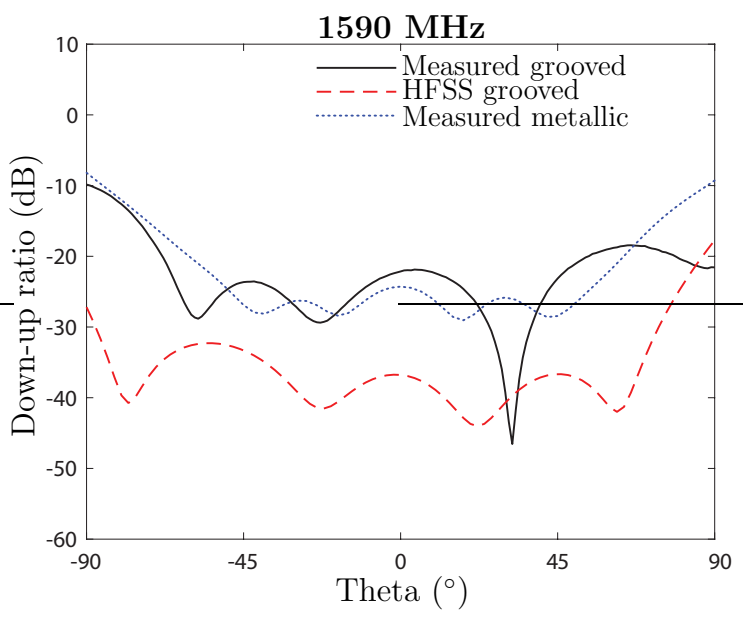

(c)

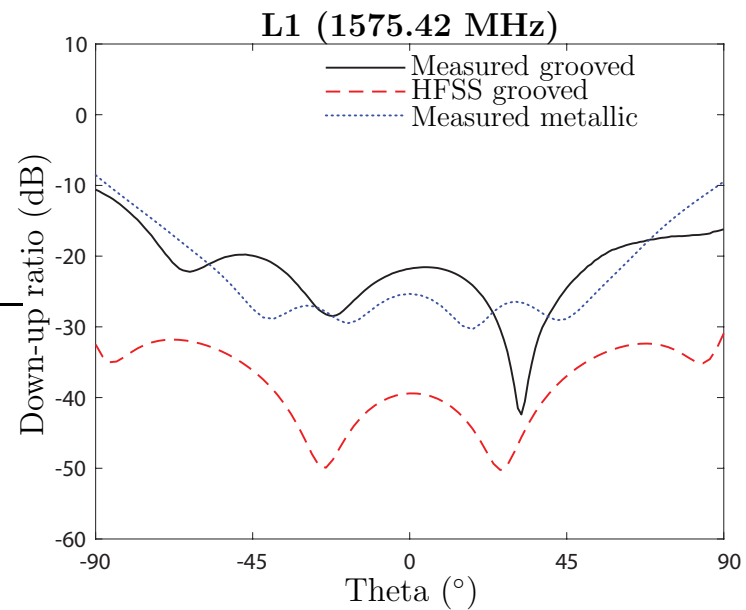

(b)

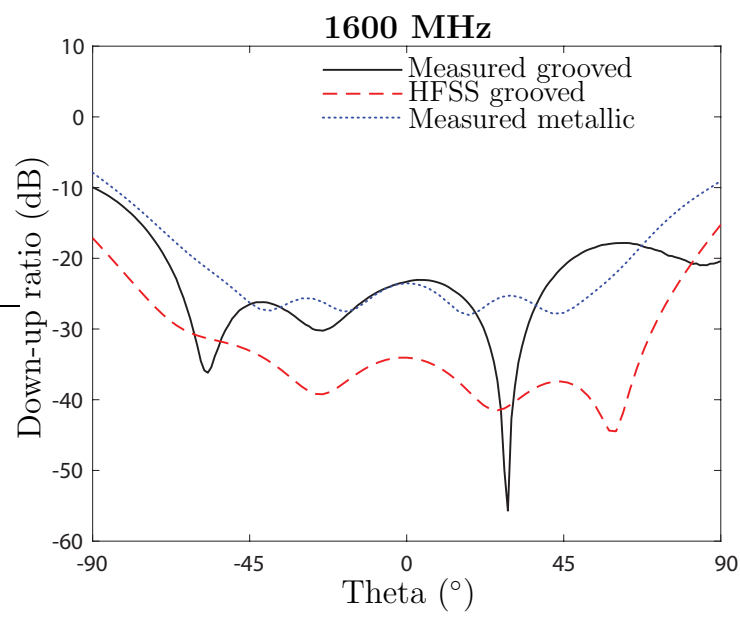

(d)

Figure 2.20: Comparison between the down-up ratio of the proposed design (experiment \& FEM-HFSS simulation) and the conventional metallic choke ring (experiment) at L1 and various other frequencies around the L1 band a) $1560 \mathrm{MHz}$, b) L1 (1575.42 $\mathrm{MHz}$, c) $1590 \mathrm{MHz}$, and d) $1600 \mathrm{MHz}$. 


\subsubsection{Discussion}

As was mentioned in Sec. 2.4.1, the conventional metallic choke ring is optimized for the lower L2 band, while offering practically good surface wave suppression capabilities around L1 band. The proposed grooved dielectric structure sacrifices the optimized performance at the L2 band, while maintaining good performance in both L1 and L2 bands, offering smaller dimensions and lighter weight. This is the main tradeoff involved in this structure. It should be noted that this performance is naturally superior to other single-resonant dielectric based structures which are dominantly optimized for only one band (L2 or L1).

Finally, the measured axial ratios of the proposed grooved dielectric structure and that of the metallic choke ring are shown in Fig. 2.21. While the proposed design exhibits better axial ratio at the higher band frequencies, with respect to the metallic choke ring, the lower band is found to exhibit relatively higher axial ratio, particularly at the L2 frequency. This can be attributed to the high fabrication tolerances in the rudimentary in-house prototype, where due to the resonant nature of the structure, any fabrication error in the height may shift the operation frequency from the desired capacitive region to the undesired inductive region. This is suggested by the improved axial ratios at $1260 \mathrm{MHz}$ and $1270 \mathrm{MHz}$, as compared to that of L2 frequency. To demonstrate this, the axial ratio was simulated for the proposed design at the design frequency L2 (1227.60 MHz) and two surrounding frequencies around the resonance (1200 MHz \& $1260 \mathrm{MHz}$ ), as shown in Fig. 2.22. It is seen that the axial ratio at L2 and $1260 \mathrm{MHz}$ is low due to the frequencies falling on the capacitive side of surface impedance. On the other hand, the axial ratio is high at $1200 \mathrm{MHz}$ due to the high inductive surface impedance. The high axial ratio measured at L2 can be remedied in the future by device prototyping using standard PCB processes including continuous

copper plating instead of copper nails, and accurate radiation pattern measurements in the anechoic chamber. 


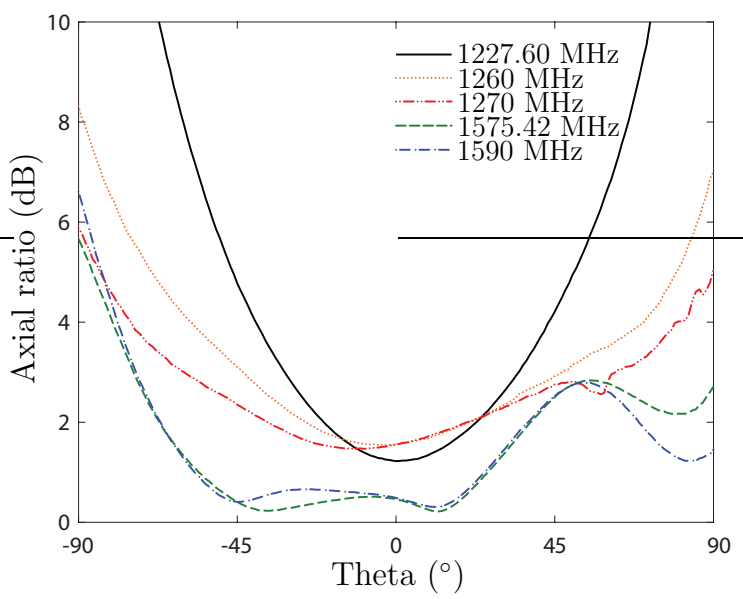

(a)

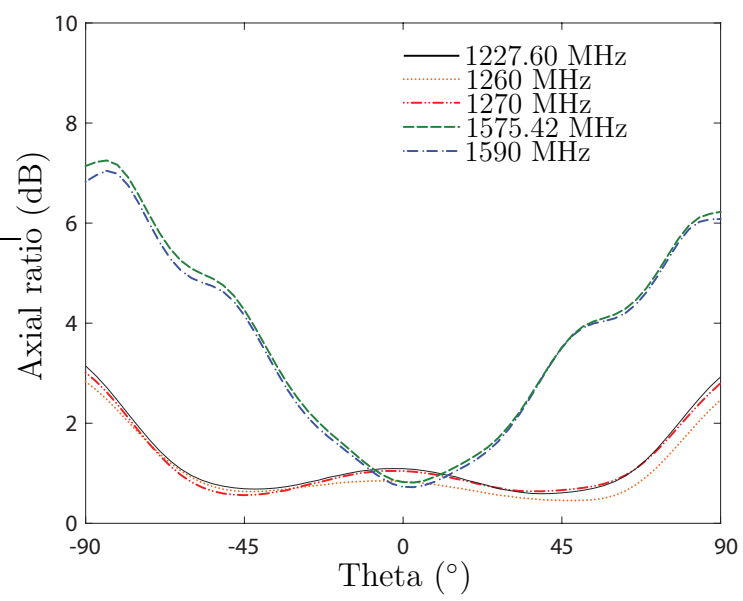

(b)

Figure 2.21: Measured axial ratio of a) the proposed design, and b) the conventional choke ring.

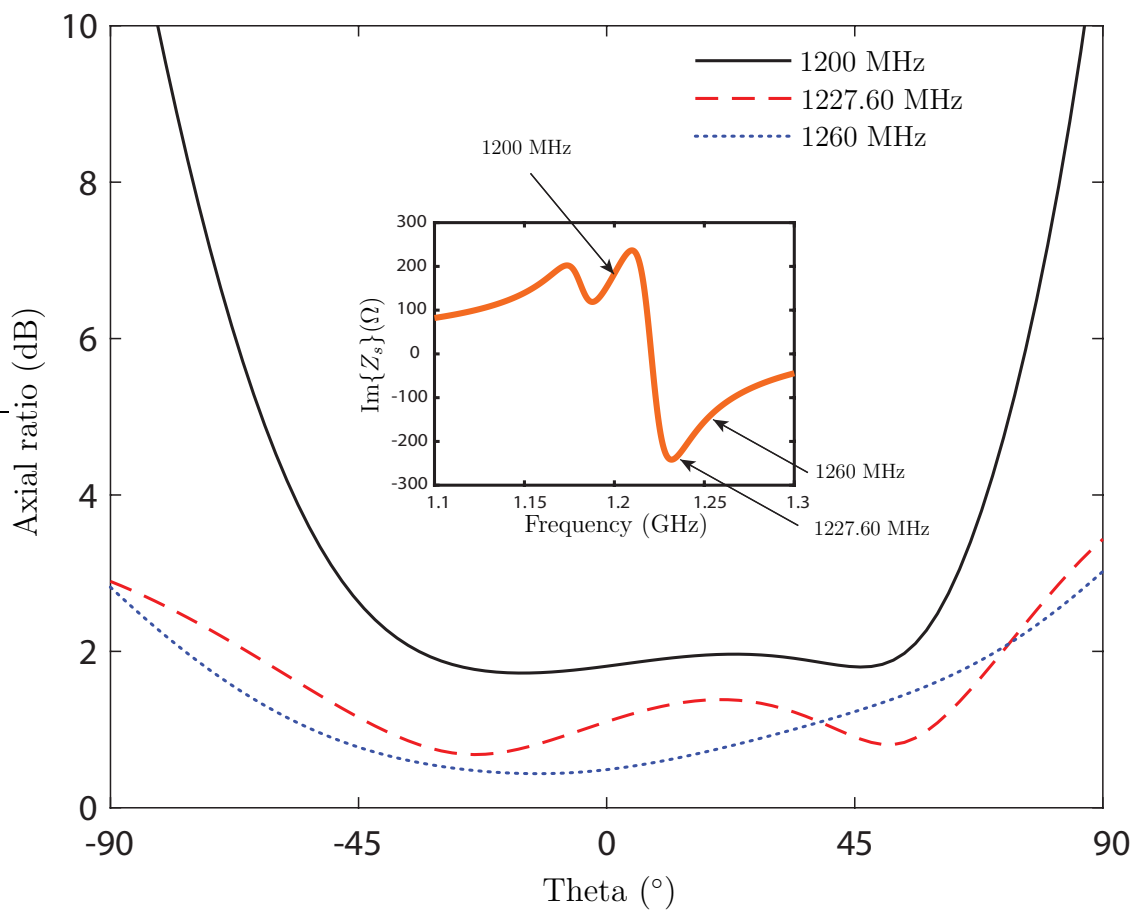

Figure 2.22: FEM-HFSS demonstration of effect of surface impedance on axial ratio. 


\subsubsection{Conclusion}

A novel low-profile grooved dielectric choke ring with dual-band surface impedance resonance has been proposed and experimentally demonstrated. The proposed structure is based on engineering the surface impedance of the dielectric based choke ring, by strategically introducing two resonant frequencies around the GNSS bands of L2 and L1. This has been shown to provide capacitive surface impedances around both bands simultaneously, required for efficient surface wave suppression for higher multipath rejection performance. An in-house choke ring prototype of the proposed design has been tested with the DM antenna and has shown high multipath rejection performances in both GNSS bands, comparable to an otherwise bulky, heavy, and expensive conventional metallic choke ring. The proposed design further provides $58 \%$ reduction in height and 26\% reduction in diameter using the FR4 material employed, and is an attractive low-cost solution for high performance GNSS applications. 


\section{Chapter 3}

\section{Active Phasers for Analog Signal Processing Applications}

\subsection{Background}

While the previous chapter focused on surface impedance engineering for radiative applications, dispersion engineering can also be used for guided-wave applications. This will be the focus of this chapter.

Digital signal processing (DSP) has been the predominant paradigm for processing signals at low frequencies due to its flexibility, reliability, and compactness. However, at high frequencies, digital technologies begin to suffer from high complexity, reduced performance, and high power consumption, due to the challenging implementation of analog-to-digital (ADC) and digital-to-analog converters (DAC) [86] [87]. To overcome these challenges, real-time analog signal processing (R-ASP) has reemerged as an alternative to DSP for processing signals at the mm-wave frequencies without relying on conventional digital techniques [85]. The fundamental components of such systems are dispersion engineered devices, whose group delay is designed according to a specified function of frequency, suitable for a given specific application. These devices are known as phasers.

Group delay dispersion in phasers is achieved using coupled transmission lines. Two types of coupling can occur when two or more transmission lines are brought close together: contra-directional coupling (or backward coupling), and co-directional coupling (or forward coupling). Contra-directional couplers operate within the realm of TEM wave propagation and are suitable for low frequency applications using microstrip transmission lines. Co-directional coupling is a concept borrowed from optics which is based on non-TEM wave propagation and hence it is suitable for high frequency applications where substrate-integrated waveguides (SIW) can be used as the transmission lines. 
While various phasers have been demonstrated at the microwave frequencies, very little work has been done in developing high performance phasers in the higher frequency bands (typically more than the X-band). This is of particular relevance as the real-time signal processing technology is more suitable for the mm-wave bands, where the digital technologies begin to suffer from high complexity, reduced performance, and high power consumption [86] [87].

In various signal processing applications, such as real-time Fourier transformers (RTFT) and frequency discriminators, large group delay dispersion is typically required in a large bandwidth, i.e. large group delay-bandwidth product. Moreover, following Kramers-Kronig relations, a strong dispersion leads to strong frequency dependent transmission magnitude, which is an unfavorable and an undesired feature of a phaser [88]. The focus of this thesis is to design active phasers based on contradirectional couplers, which are suitable for microwave frequency operation, and active phasers based on co-directional couplers, which are suitable for mm-wave frequency operation. Active phasers would provide a strong dispersive response without transmission magnitude distortions.

\subsection{Proposed Active Microwave C-Section}

\subsubsection{Contra-directional Couplers based Phaser}

Low frequency phasers are based on contra-directional TEM coupled-line couplers [90] as shown in Fig. 3.1(a). A microwave C-section is a 2-port device designed by connecting the two ends of the coupled lines together as shown in Fig. 3.1(b). A Csection represents a fundamental building unit of microwave dispersive devices such a wide-band phase shifters [89], equalizers [90] and real-time signal processors [91][92]. Recently, they have been used extensively to build dispersive devices exhibiting complex group delay profiles, owing to their all-pass transmission properties, which have found important applications in R-ASP technology [93].

Under ideal lossless conditions, a C-section provides a flat transmission $\left[\left|S_{21}(\omega)\right|=\right.$ $1]$ with a strongly dispersive frequency-dependent group delay response $[\tau=\tau(\omega)]$. However, all practical devices exhibit dissipation losses, and therefore, a flat transmission is never achieved in practice, i.e. $\left|S_{21}(\omega)\right| \neq 1$. Its magnitude transmission is always dependent on the dispersion response following Kramers-Kronig relations (3.1) and the corresponding Bode gain-phase relations (3.2) [94]. 


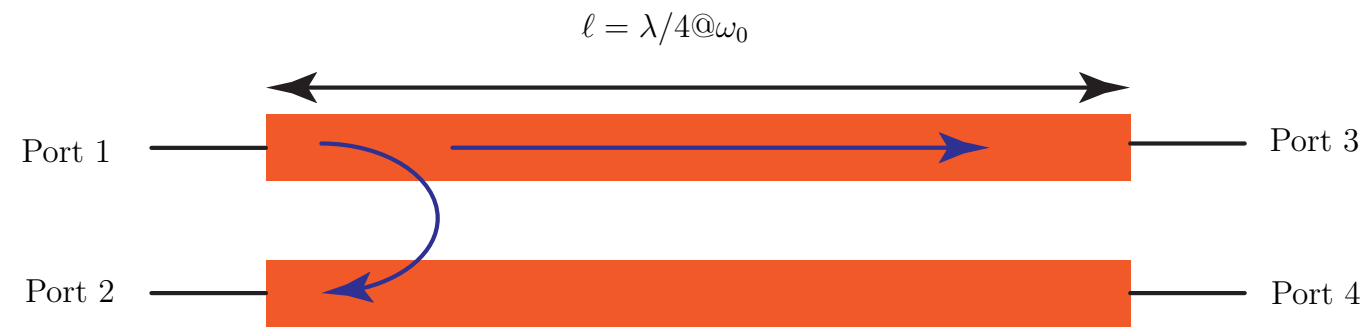

(a)

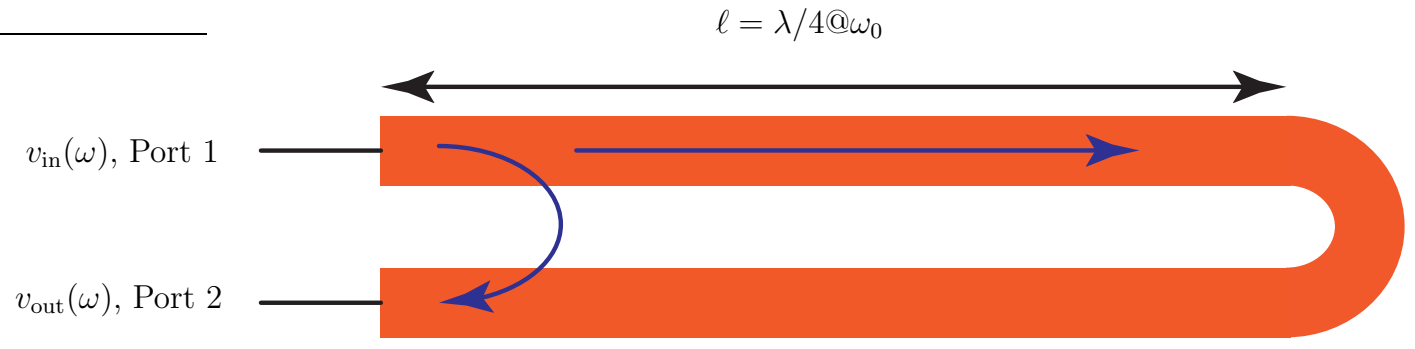

(b)

Figure 3.1: a) Contra-directional coupler, and b) microwave C-section based on contra-directional coupler.

$$
\begin{gathered}
\chi^{\prime \prime}(\omega)=\frac{2 \omega}{\pi} \int_{0}^{+\infty} \frac{\chi^{\prime}(\omega)}{\omega^{\prime 2}-\omega^{2}} d \omega^{\prime} \\
\chi^{\prime}(\omega)=-\frac{2}{\pi} \mathcal{P} \int_{0}^{+\infty} \frac{\omega^{\prime} \chi^{\prime \prime}(\omega)}{\omega^{\prime 2}-\omega^{2}} d \omega^{\prime} \\
\angle T(\omega)= \pm \frac{2 \omega}{\pi} \mathcal{P} \int_{0}^{+\infty} \frac{\ln \left\{\left|T\left(\omega^{\prime}\right)\right|\right\}}{\omega^{\prime 2}-\omega^{2}} d \omega^{\prime}
\end{gathered}
$$

This frequency-dependent magnitude transmission subsequently leads to undesired signal distortion at the output of the corresponding dispersive devices. To combat this issue, the concept of a perfect dispersive medium (PDM) was recently developed in [94] to decouple the magnitude and phase response of an electromagnetic device, while satisfying all causality requirements, i.e. achieving $\left|S_{21}(\omega)\right|=1$ and $\tau=\tau(\omega)$, simultaneously. The 1-D counterpart of a PDM was demonstrated using microwave C-sections in [95], where each super-cell consisted of two separate loss and gain C-sections leading to amplitude-equalization of the super-cell and dispersion reconfigurability. While dispersion reconfigurability is specific to certain systems such as dispersion code multiple access (DCMA), amplitude equalization is a desired characteristic of any other dispersion based system such as real-time Fourier transformers [96][97], spectrum sniffers or frequency discriminators [98].

In this work, the same flat transmission response and frequency-dependent group 
delay are achieved simultaneously using a single microwave C-section. It will be shown that the losses of the $\mathrm{C}$-section can be perfectly compensated in a wide band by integrating a gain-load inside a microwave C-section.

The analytical transfer function of a microwave C-section is given by [99][100]

$$
S_{21}(\theta)=\left(\frac{1-j \rho \tan \theta}{1+j \rho \tan \theta}\right)
$$

where $\rho=\sqrt{(1-k) /(1+k)}$, with $k$ as the voltage coupling coefficient and $\operatorname{Re}\{\theta(\omega)\}=$ $\gamma(\omega) \ell$ as the electrical length of the transmission with $\gamma$ as the complex propagation constant of the isolated line. The complex propagation constant $\gamma(\omega)=\beta(\omega)-j \alpha(\omega)$, where $\beta$ and $\alpha$ are the per-unit propagation and attenuation constants of the isolated transmission lines, respectively. For a practical C-section with $\alpha \neq 0$, the transfer function can be re-written as [95]

$$
S_{21}(\omega)=\left\{\frac{1-\rho \tanh \alpha \ell-j(\rho-\tanh \alpha \ell) \tan \beta \ell}{1+\rho \tanh \alpha \ell+j(\rho+\tanh \alpha \ell) \tan \beta \ell}\right\},
$$

It can be verified that the transmission magnitude $\left|S_{21}(\omega)\right| \neq 1$, and is a strong function of frequency. In addition, the minimum transmission, $\min \left\{\left|S_{21}(\omega)\right|\right\}$, is at the resonant frequency $\omega_{0}$ of the coupler where its length $\ell$ is a quarter wavelength long.

A C-section also exhibits a strong dispersion (frequency dependent group delay). Its group delay response $\tau(\omega)=-d \angle S_{21}(\omega) / d \omega$. It can be easily verified that the maximum group delay $\tau$ occurs at the resonant frequency $\omega_{0}$ of the coupler, and is given by

$$
\max \{\tau(\omega)\}=\frac{2}{\rho} \frac{d \beta(\omega)}{d \omega} \ell
$$

\subsubsection{Proposed Active Phaser}

To achieve a flat magnitude transmission from a microwave C-section while preserving its strongly dispersive characteristic, let us modify the microwave C-section where the through and the isolated ports of the corresponding coupler are now connected through a matched load $G$, as shown in Fig. 3.2. Using the wave-interference approach, the transfer function of such a $\mathrm{C}$-section is given by 


$$
S_{21}(\theta)=a(\theta)+\frac{G \cdot b^{2}(\theta)}{1-a(\theta) \cdot G}
$$

where $a(\theta)$ and $b(\theta)$ are the backward coupling and through responses, respectively. When $G=1,(3.6)$ corresponds to a passive C-section, and reduces to (3.4), with $\left|S_{21}(\omega)\right| \neq 1$. This expression suggests that the load parameter $G$ acts as a free parameter which maybe used to compensate for the frequency-dependent losses in a practical C-section. Let us intuitively enforce the amplitude-equalization at the fundamental resonant frequency $\omega_{0}(\beta \ell=\pi / 2)$ of the coupler, i.e.

$$
a(\pi / 2-j \alpha \ell)+\frac{G \cdot b^{2}(\pi / 2-j \alpha \ell)}{1-a(\pi / 2-j \alpha \ell) \cdot G}= \pm 1
$$

so that $\left|S_{21}\left(\omega_{0}\right)\right|=1$. The \pm signs on the RHS corresponds to resonant or antiresonant conditions exhibiting the two group delay extrema. This equation can be easily solved for an optimum value of $G=G_{0}$, in closed-form to achieve a unity transmission across the $\mathrm{C}$-section at the resonant frequency.

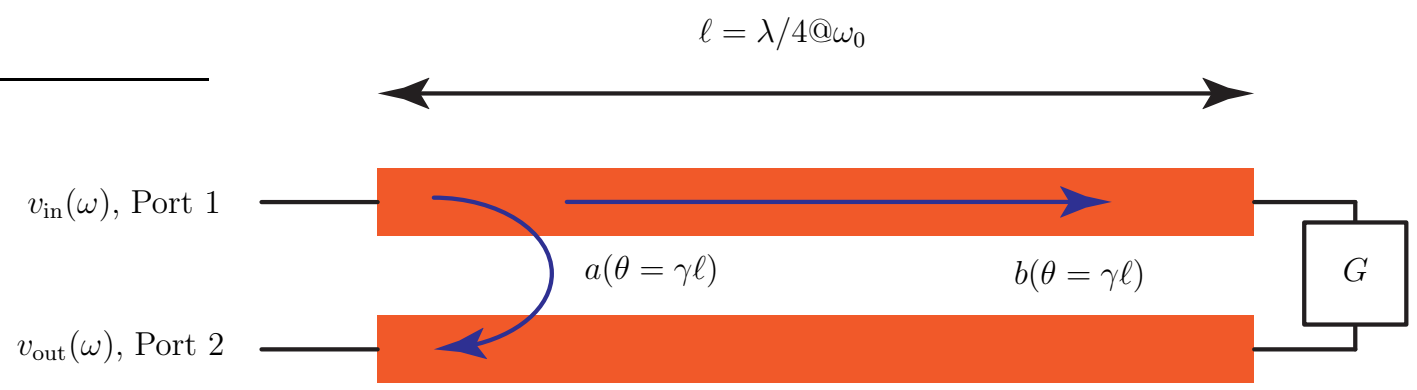

Figure 3.2: Proposed configuration of an amplitude-equalized microwave C-section, where the through and isolated ports of the corresponding coupler are connected through a load $G$.

Note that in the previous analysis, the losses and the integrated gain, $G$, are assumed to be constant with respect to frequency, while in practical implementations of the active C-section, the gain will be frequency-dependent. Under these circumstances, a perfect flat transmission may not be achieved.

Fig. 3.3 shows the modified magnitude and group delay responses of the amplitudeequalized C-section, following the optimum value of $G_{0}$. The transmission magnitude is now perfectly equalized to $0 \mathrm{~dB}$. Naturally, the C-section maintains a strong dependence of the group delay on frequency, as desired. Fig. 3.4 confirms this feature of an active C-section, using full-wave simulations in FEM-HFSS, for a stripline structure (not shown here). The optimal gain load required agrees with the numerical value from (3.7), thereby confirming the operation of the active C-section. 

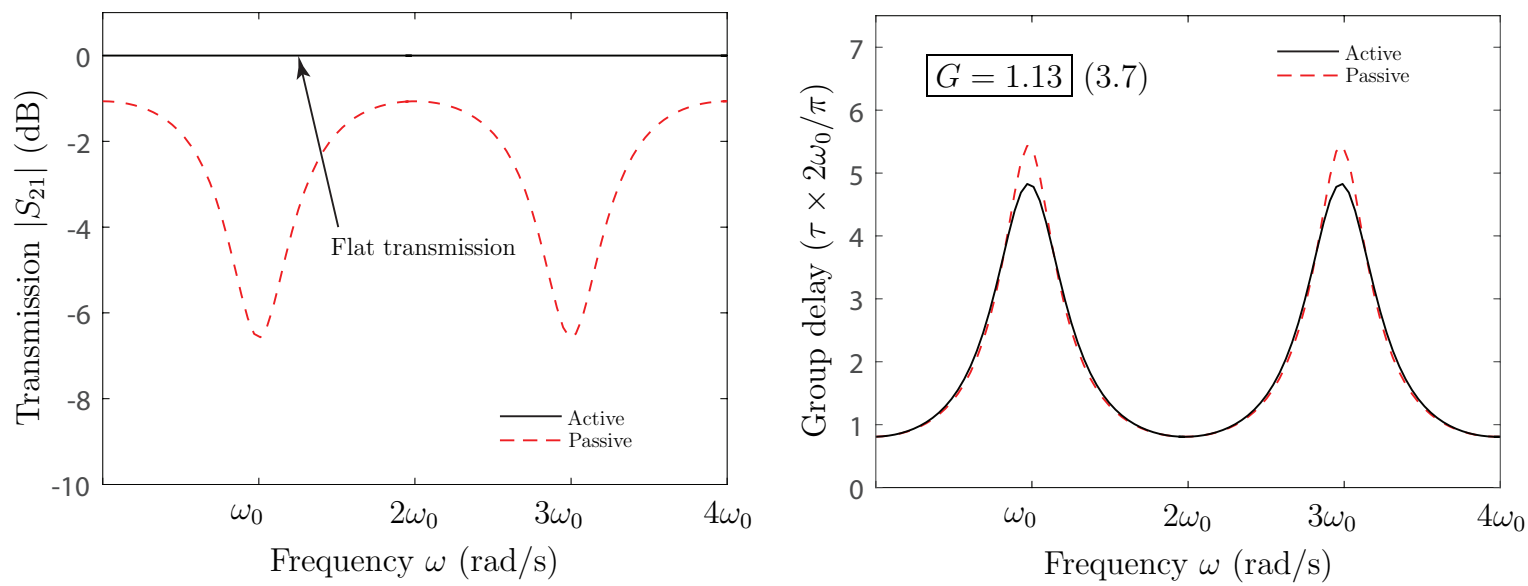

Figure 3.3: The magnitude transmission and group delay responses of an active microwave C-section of Fig. 3.2 computed using (3.6). The responses of a passive Csection is also shown using dashed curves for comparison. The design parameters are: $k=0.71, \alpha \ell=0.15$ and the gain $G_{0}$ calculated using (3.7).
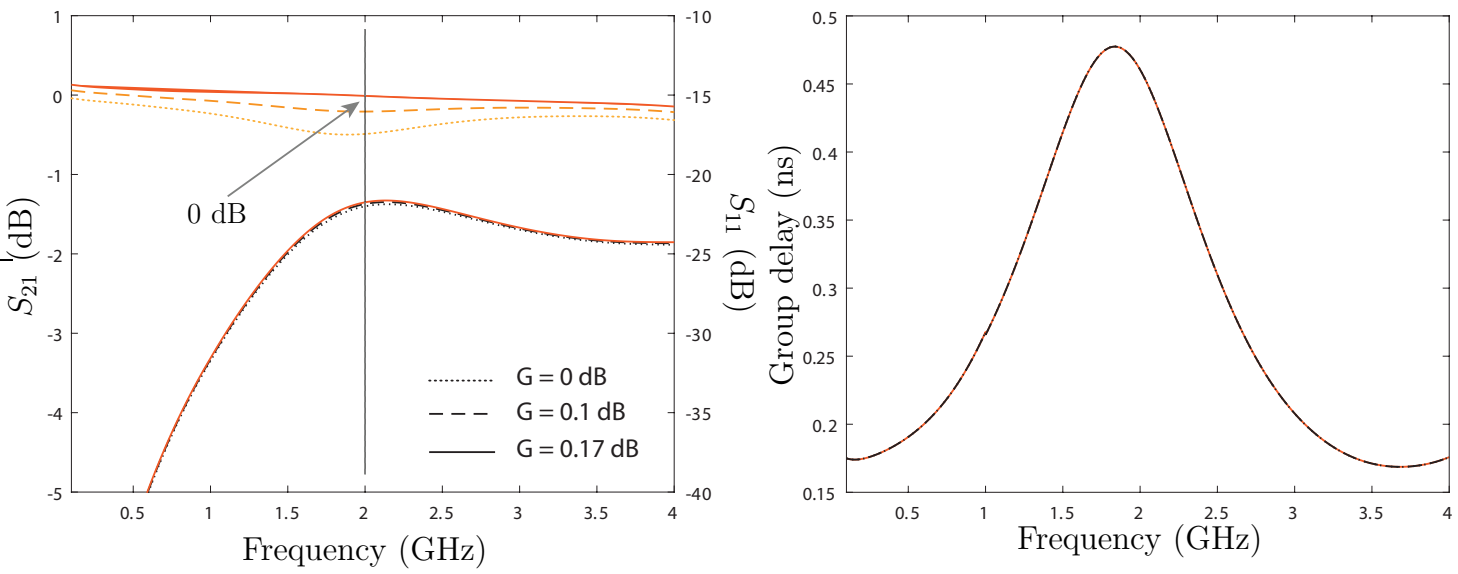

Figure 3.4: FEM-HFSS Simulated S-parameters and group delay response of an active C-section implemented in stripline technology, for different values of Gain load, including the optimum value $G_{\mathrm{opt}}=0.17 \mathrm{~dB}$. 


\subsection{Proposed Active Millimeter-Wave Phaser}

\subsubsection{Co-directional Couplers based Phaser}

In order to implement phasers at mm-wave frequencies, co-directional coupling must be chosen over contra-directional coupling. As mentioned earlier, co-directional coupling operates with non-TEM wave propagation so they can be implemented using substrate integrated waveguide (SIW) structures. SIW structures are more practical than microstrip lines at higher frequencies due to their low loss.

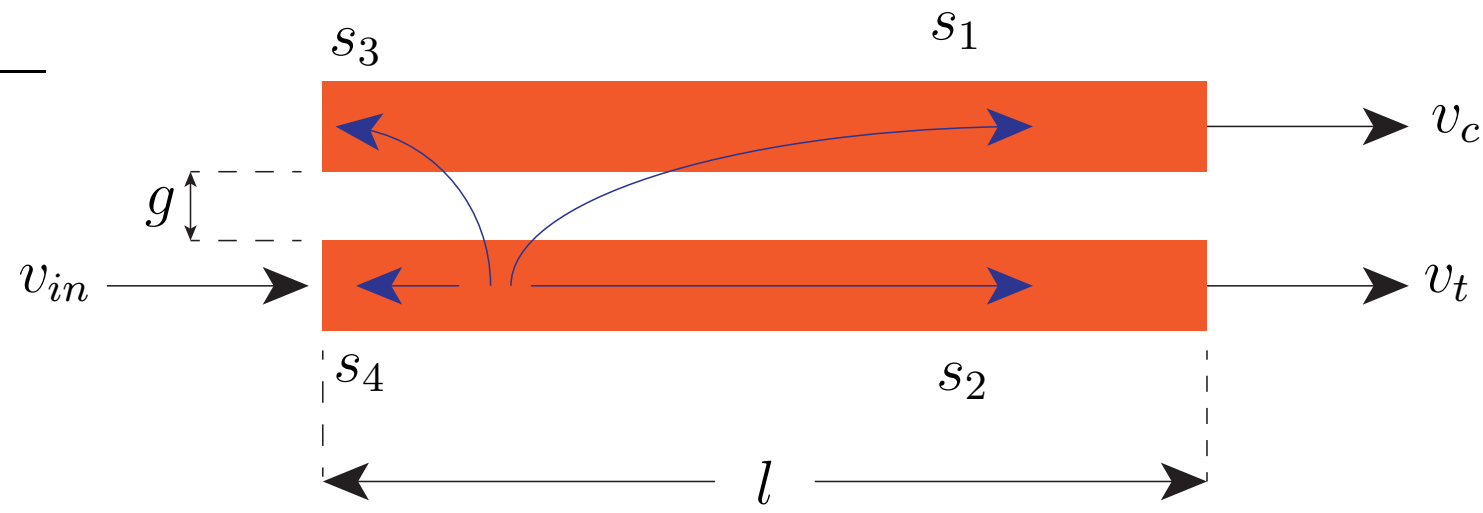

Figure 3.5: Co-directional coupler with length, $l$ and gap, $g$. The co-directional coupling is $s_{1}$, and through is $s_{2}$. In an ideal co-directional coupler, contra-directional coupling, $s_{3}=0$, and reflection, $s_{4}=0$.

A co-directional coupler is a four-port device as shown in Fig. 3.5. Analytical treatment of co-directional couplers can be found in [2]. The transmission matrix for a coupler can be written as follows:

$$
T=\left[\begin{array}{ll}
A(l) & B(l) \\
C(l) & D(l)
\end{array}\right]=\left[\begin{array}{cc}
\cos (\kappa l) & -j \sin (\kappa l) \\
-j \sin (\kappa l) & \cos (\kappa l)
\end{array}\right]
$$

from which the forward coupled and through responses can be extracted, as

$$
\begin{gathered}
s_{1}=-j \sin (\kappa l) e^{-j \gamma l} \\
s_{2}=\cos (\kappa l) e^{-j \gamma l}
\end{gathered}
$$

where $l$ is the coupled length [m], $\kappa$ is coupling per unit length $[\mathrm{rad} / \mathrm{m}]$, and $\gamma=\beta-j \alpha$ is the complex propagation constant. The gap between the two lines and the coupled length, $l$, determine the coupling coefficient. In an ideal forward coupler, the reflection $s_{4}$ and isolation $s_{3}$ are both zero. 


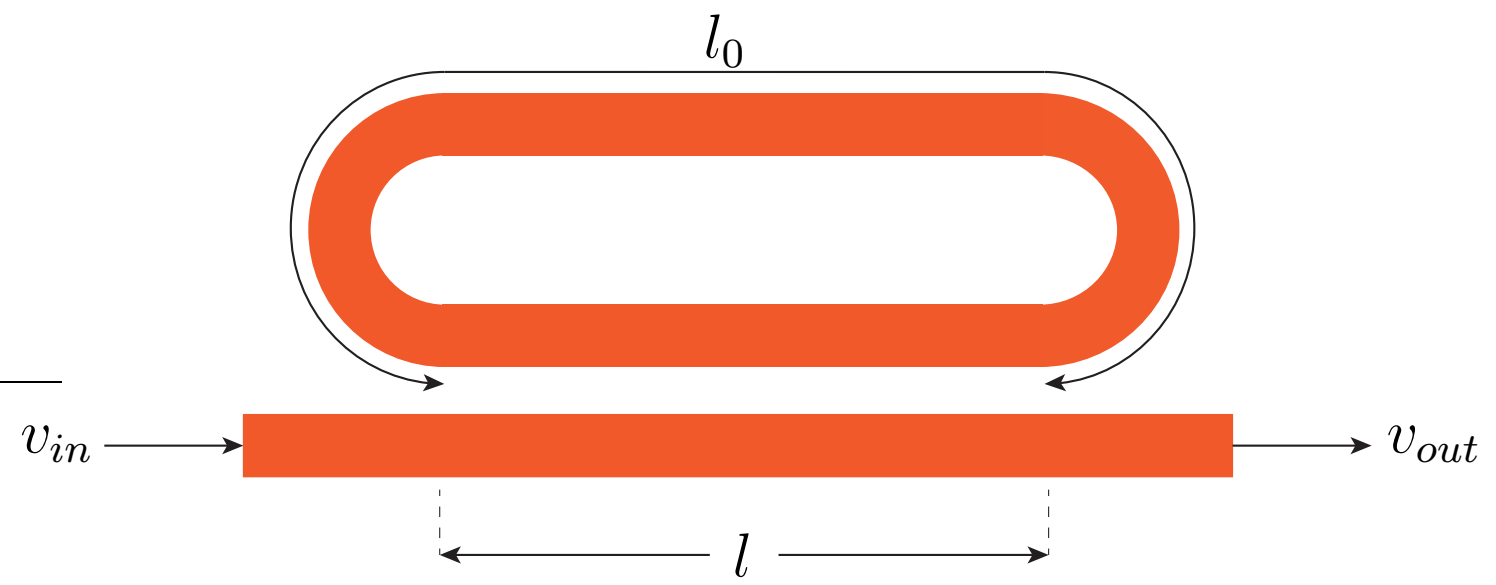

Figure 3.6: Phaser based on co-directional coupler formed by making a ring from one section of the coupler.

A two-port phaser can be formed by connecting the ends of one of the coupled lines together to make a ring as shown in Fig. 3.6. The transmission response of the phaser can be formed by summing up the through and around-the-ring responses of the phaser as follows:

$$
\begin{aligned}
T(\omega) & =s_{2}+s_{1} e^{-j \gamma l_{0}} s_{1}+s_{1} e^{-j \gamma l_{0}} s_{2} e^{-j \gamma l_{0}} s_{1}+\ldots \\
& =s_{2}+\frac{s_{1}^{2} e^{-j \gamma l_{0}}}{1-s_{2} e^{-j \gamma l_{0}}}
\end{aligned}
$$

The first expression in the equation is the through response, the second expression is due to the first turn around the ring, and the third is due to the second turn around, and so on. In this equation, $l_{0}$ is the total length of the phaser ring (minus the coupled length, $l$ ), as shown in Fig. 3.6(a). The corresponding group delay is given by,

$$
\tau_{g}(\omega)=-\frac{d \phi_{T}(\omega)}{d \omega}
$$

where $\phi_{T}$ is the phase of the transmission coefficient $T(\omega)$.

Fig. 3.7, Fig. 3.8, and Fig. 3.9 show parametric studies of the effects of $l_{0}, k$, and $\alpha$, respectively, on the transmission and group delay responses of a phaser. Fig. 3.7 shows that the length of the ring determines the resonant frequency of the phaser with no effect on group delay or transmission. Fig. 3.8 shows that the coupling coefficient, which is determined by the coupled length and the gap, controls the group delay and transmission characteristics of the phaser. Finally, Fig. 3.9 shows that attenuation in the phaser affects the transmission response with negligible effect on the group delay, with the ideal lossless case having a zero flat transmission response, as expected. 


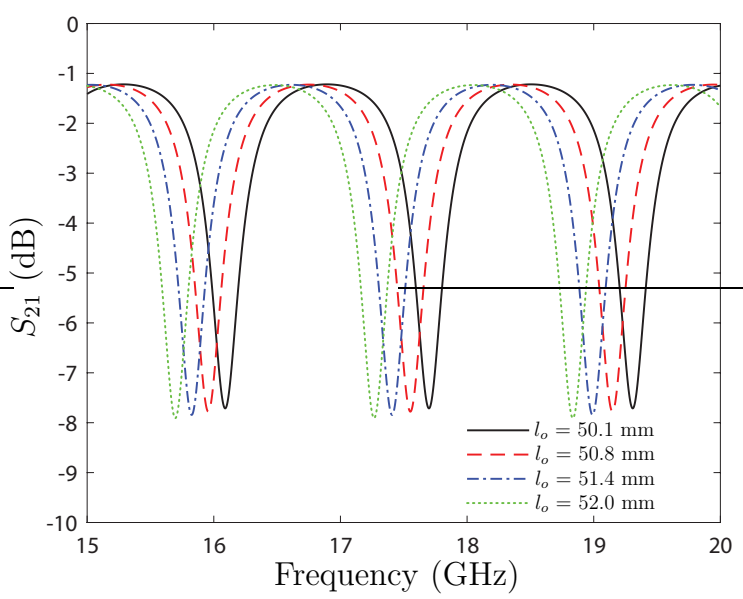

(a)

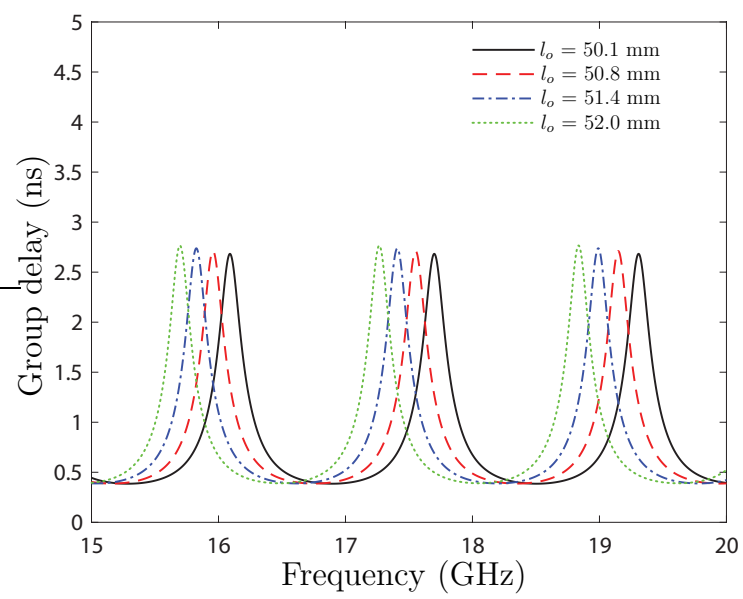

(b)

Figure 3.7: Parametric study of the effect of ring length on the phaser response. a) Transmission as a function of frequency, and b) group delay as a function of frequency, with various ring lengths.

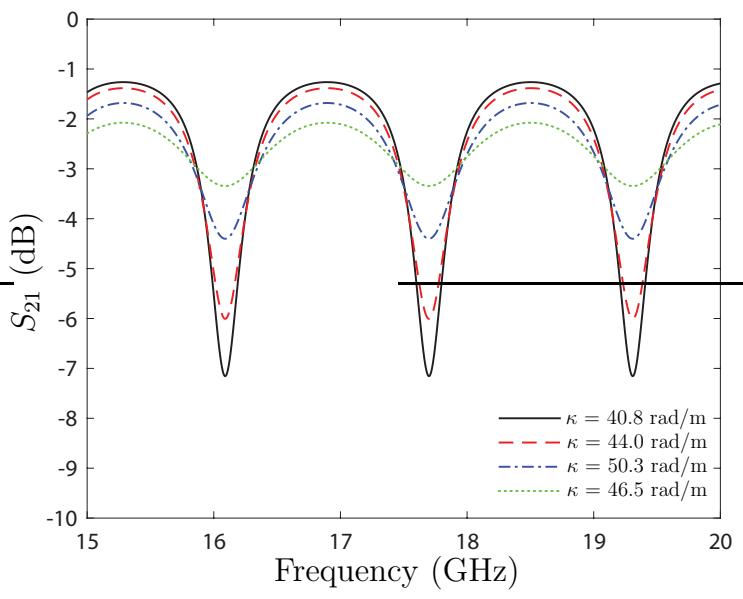

(a)

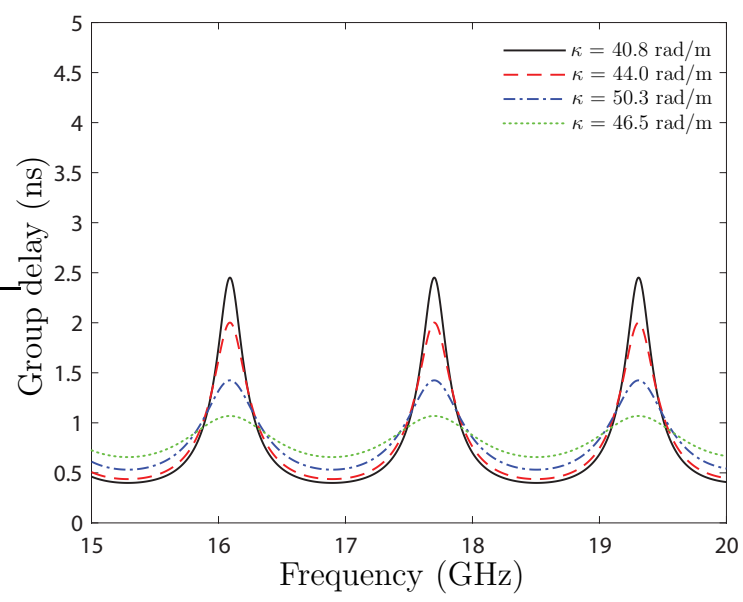

(b)

Figure 3.8: Parametric study of the effect of coupling coefficient on the phaser response. a) Transmission as a function of frequency, and b) group delay as a function of frequency, with various coupling coefficients. 


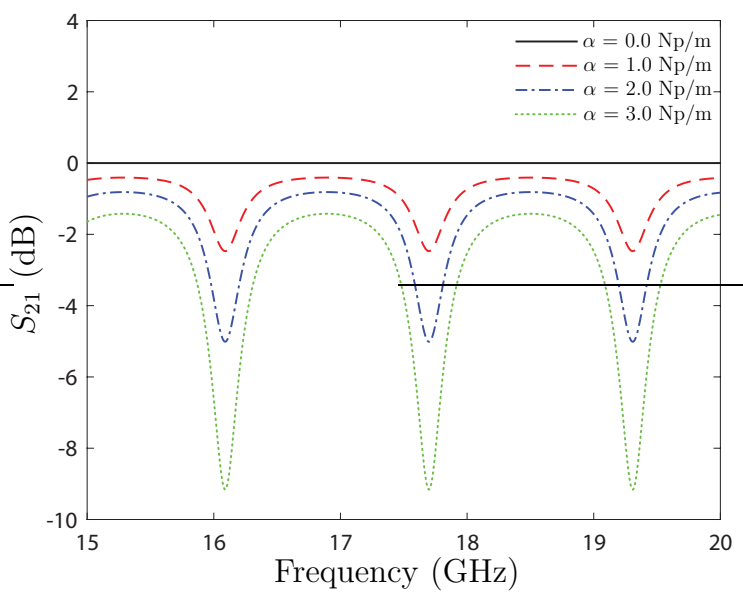

(a)

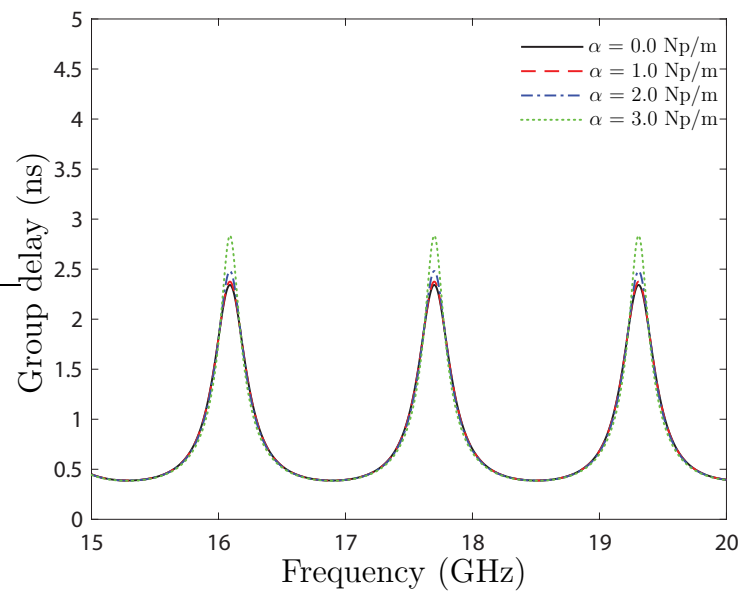

(b)

Figure 3.9: Parametric study of the effect of attenuation constant on the phaser response. a) Transmission as a function of frequency, and b) group delay as a function of frequency, with various attenuation constants. 


\subsubsection{Proposed Active Phaser}

While the phaser shown above is suitable for high-frequencies due to its non-TEM nature, its frequency dependent group delay inherently leads to strong transmission magnitude distortions, following the Kramers-Kronig relations. In particular, the effect of this is a significant drop in transmission magnitude at the group delay resonant frequency. To solve this problem the proposed design involves adding a gain element inside the phaser ring as shown in Fig. 3.10 to compensate for dissipation losses and hence achieving a perfectly flat transmission response while preserving the group delay dispersion [101].
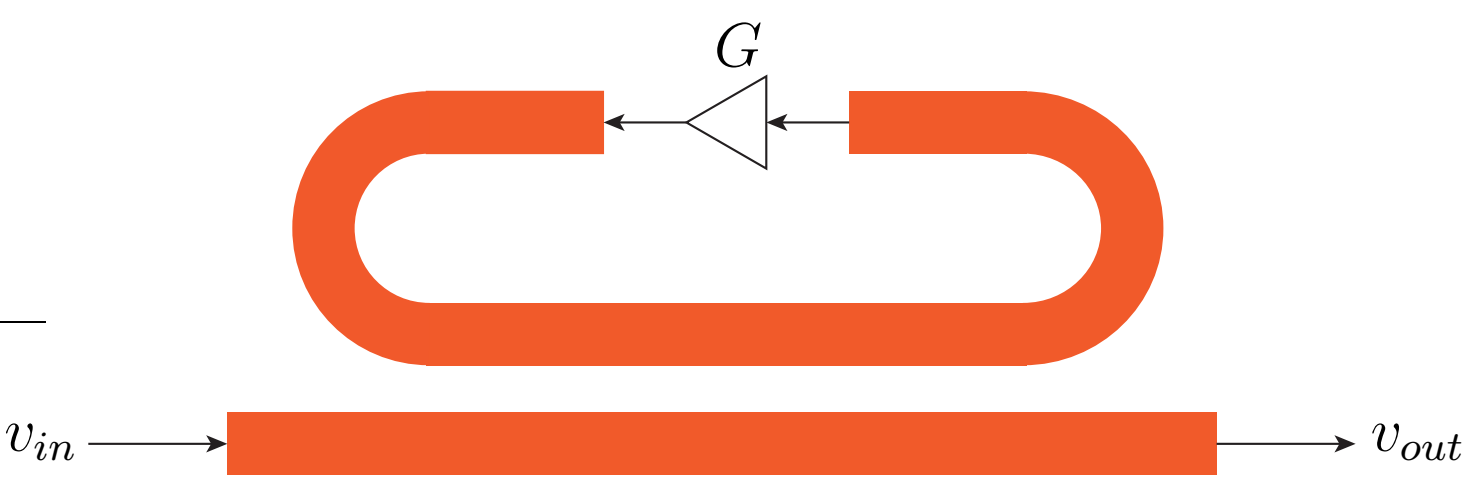

Figure 3.10: Proposed active phaser design involving adding a gain element inside the phaser ring to achieve a flat transmission response.

Adding the gain element with gain, $G$, inside the phaser ring modifies the transmission response of the phaser to

$$
T=s_{2}+\frac{s_{1}^{2} e^{-j \gamma l_{0}}}{1-G s_{2} e^{-j \gamma l_{0}}}
$$

A parametric study was conducted to investigate the effect of the gain element on the transmission and group delay responses of the phaser, as shown in Fig. 3.11. It is seen that with a specific value of gain, the transmission response can ideally be flattened across the entire bandwidth with a minimal effect on the group delay response. Note that even though the transmission response is flat, it is not perfectly lossless due losses in the through coupled section of the structure which are not compensated for with the gain element in the loop. These losses can however be compensated using an additional gain element at the output of the phaser. 


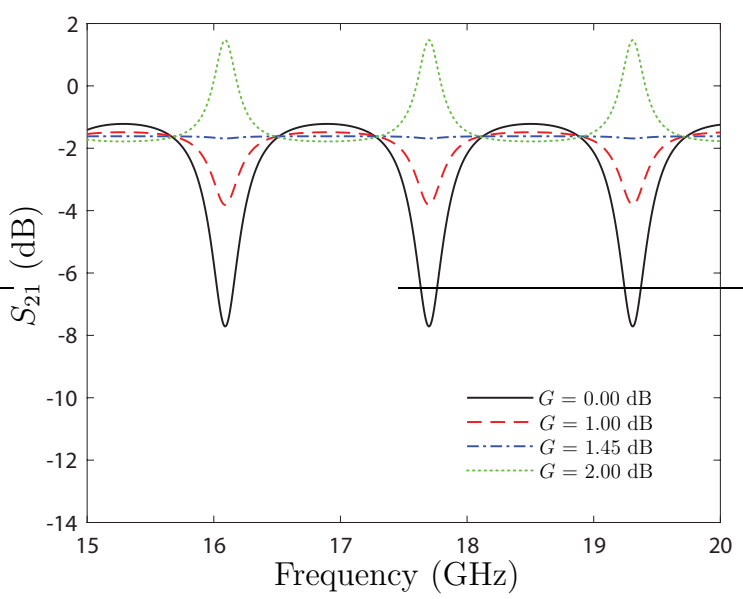

(a)

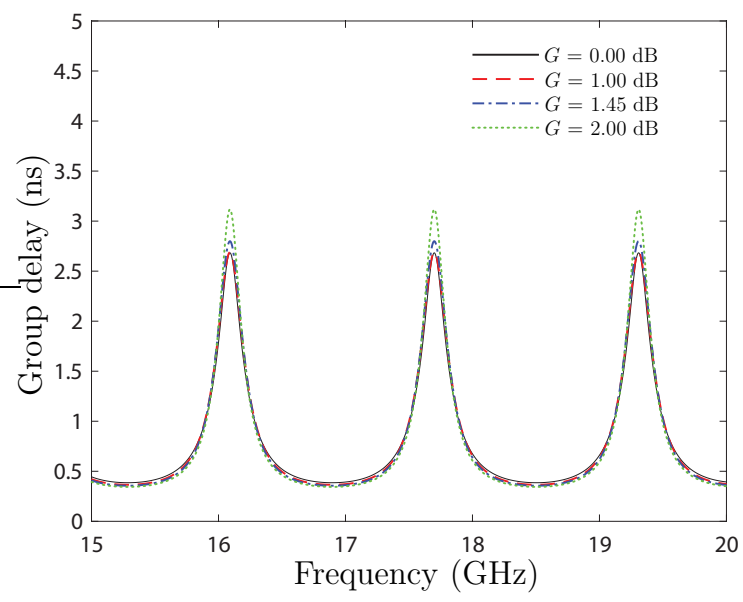

(b)

Figure 3.11: Parametric study of the effect of gain on the phaser response of a lossy structure. a) Transmission as a function of frequency, and b) group delay as a function of frequency, with various gains integrated within the phaser ring. 


\subsection{Full-Wave Demonstration}

\subsubsection{Half-Mode Substrate-Integrated Waveguide Implemen- tation}

The proposed design was implemented using a microstrip-fed half-mode substrate integrated waveguide (HMSIW) as shown in Fig. 3.12. Substrate integrated waveguides (SIW) are formed by connecting the top layer metallic layer of a substrate to ground with vias, where the plurality of vias emulates a metallic wall [102]. By designing the via diameter and the spacing between the vias, an SIW can have the same response as an equivalent rectangular waveguide [103]. The SIW implementation was chosen for a number of reasons, mainly because it supports non-TEM wave propagation required for co-directional coupling [2]. The SIW also has better performance at higher frequencies compared to microstrip lines in terms of lower radiative and conductive losses [104]. The HMSIW is formed by cutting the SIW along with middle and discarding one half, hence having a waveguide supporting half of the mode the full SIW supports, with low attenuation and compact size, thus suitable for realizing the co-directional coupler needed here [105].

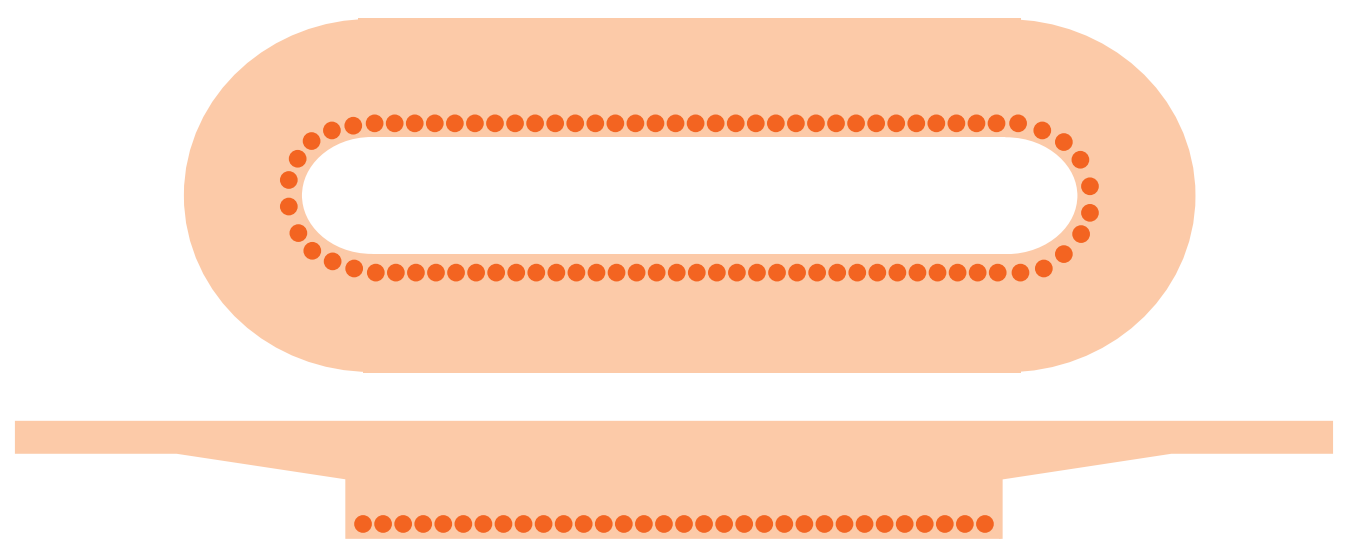

Figure 3.12: Phaser implementation using HMSIW structure.

\subsubsection{Single \& Cascaded Active Phasers}

FEM-HFSS was used to produce full-wave results for single and cascaded passive and active phaser implementations on a 25 mil thick Rogers $3006\left(\epsilon_{r}=6.15, \tan \delta=\right.$ $0.0025)$ substrate. It was decided to implement the proposed phasers in the frequency range of $16-17 \mathrm{GHz}$ as an initial proof-of-concept, with future plans to implement at mm-wave frequencies. Fig. 3.13 and Fig. 3.14 show the transmission and group delay responses of the passive and active phasers. 
As seen from Fig. 3.13(a), the passive single phaser shows a large dissipation $(>15 \mathrm{~dB})$ at the dispersive group delay frequency. This dissipation was equalized by adding a gain $G=2.8 \mathrm{~dB}$ inside the phaser ring. As seen in Fig. 3.13, the transmission magnitude was brought to nearly $0 \mathrm{~dB}$ and the group delay decreased by about 2.5 ns. For the cascaded phasers, individual phasers were equalized using the same gain and then cascaded. The result is a loss of over $30 \mathrm{~dB}$ in the passive case nearly equalized across the bandwidth in the active case, as shown in Fig. 3.14(a). Just like the single phaser, the group delay decreased and while there are three distinct peaks in the passive case, the active case shows a near linearly decreasing group delay in a $300 \mathrm{MHz}$ operational bandwidth from 16.2-16.5 GHz, as seen in Fig. 3.14(b). This characteristic is promising for RTFT applications, for instance.

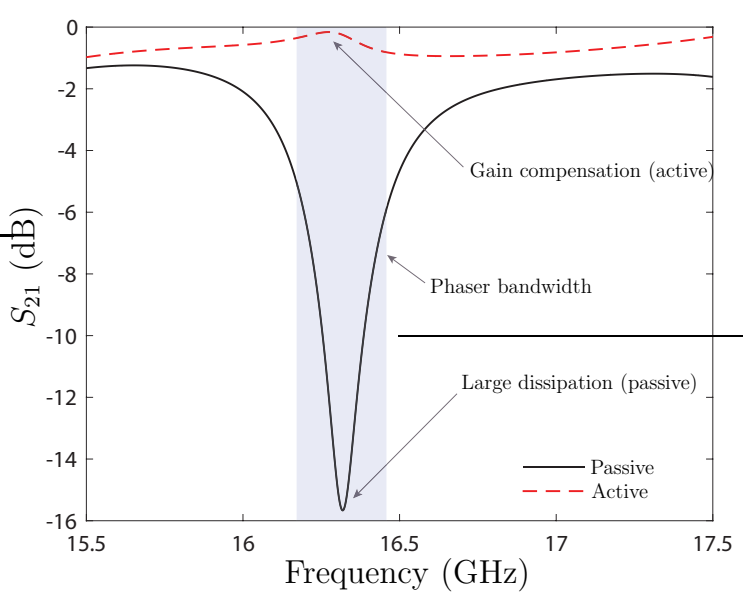

(a)

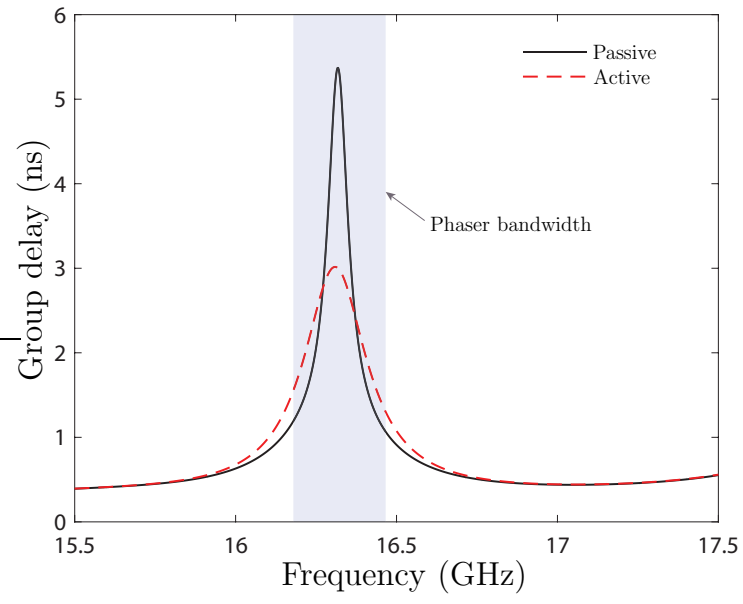

(b)

Figure 3.13: Single HMSIW phaser: comparison of passive vs. active responses. a) Transmission as a function of frequency, and b) group delay as a function of frequency.

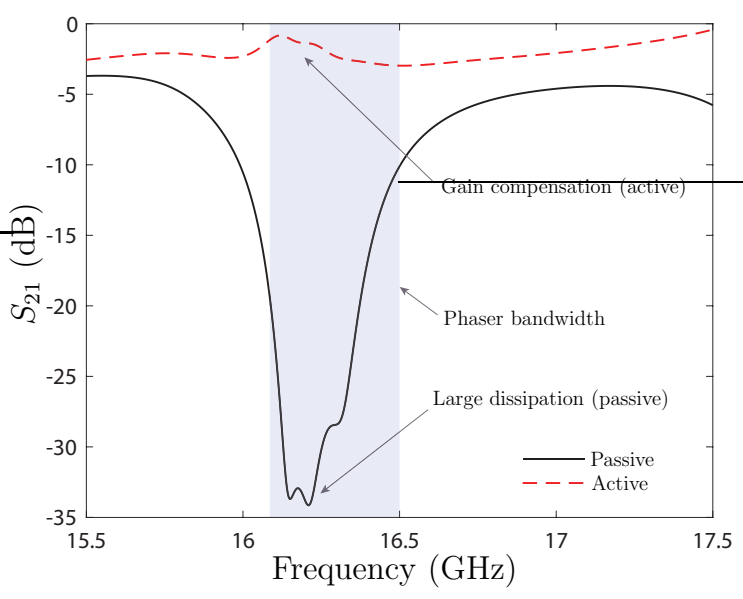

(a)

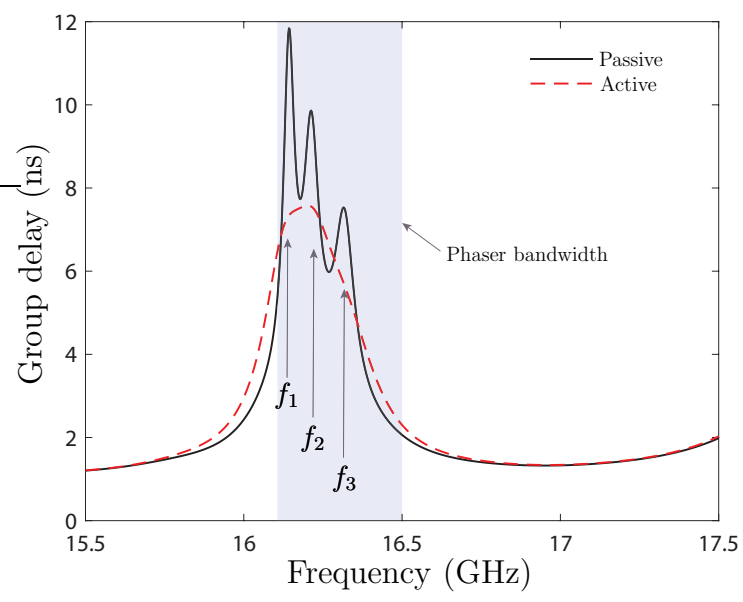

(b)

Figure 3.14: Cascaded HMSIW phaser: comparison of passive vs. active responses. a) Transmission as a function of frequency, and b) group delay as a function of frequency. 


\subsection{Experimental Demonstration}

\subsubsection{Design Prototypes}

In order to experimentally demonstrate the active phasers, a wideband $(400 \mathrm{MHz}$ to $20 \mathrm{GHz}$ ) variable gain amplifier (VGA) [106] was used as the gain element. The amplifier test board is shown in Fig. 3.15.

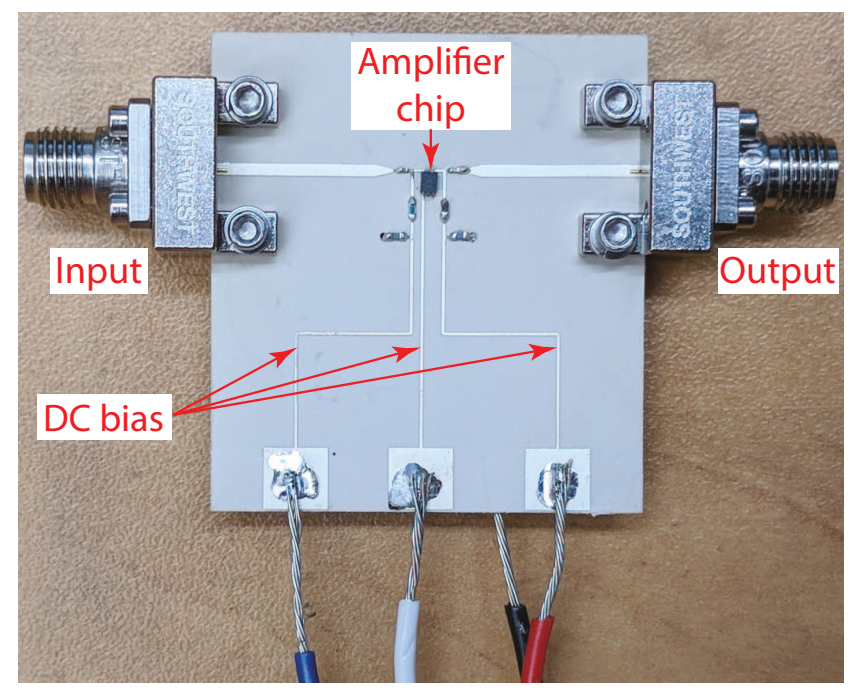

Figure 3.15: Photograph of MACOM MAAM-011100 variable gain amplifier test board.

Due to the amplifier's wideband nature, it can cause gain-peaking and oscillations at undesired frequencies. For this reason, a 4-section microstrip parallel edge-coupled bandpass filter was designed based on the concepts in [36] to act as a pre-filter for the structure. The fabricated filter is shown in Fig. 3.16.

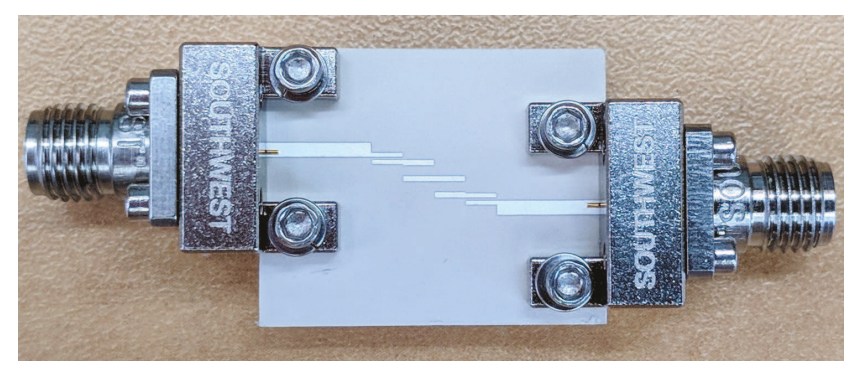

Figure 3.16: Photograph of the fabricated bandpass filter test board.

Fig. 3.17 and Fig. 3.18 show photographs of the fabricated passive and active phasers with the bandpass filter at their inputs. 


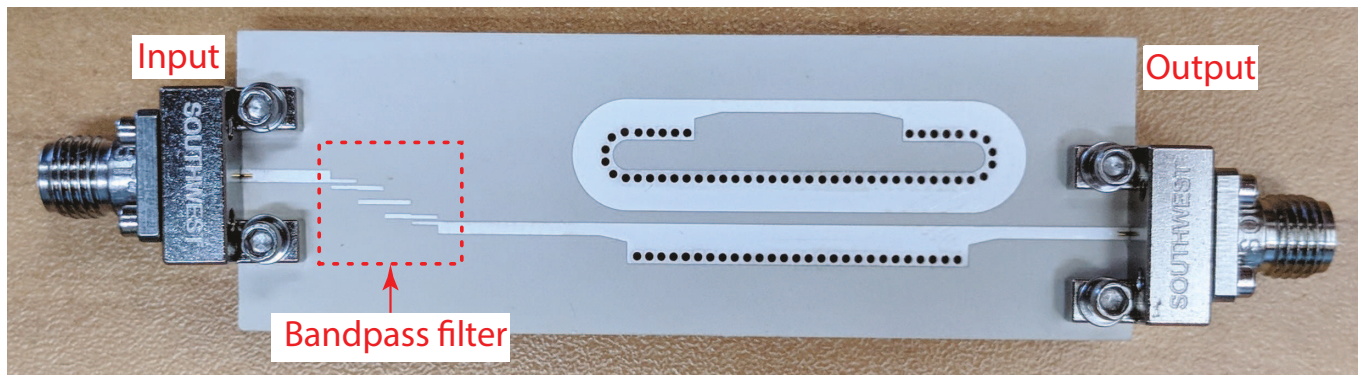

(a)

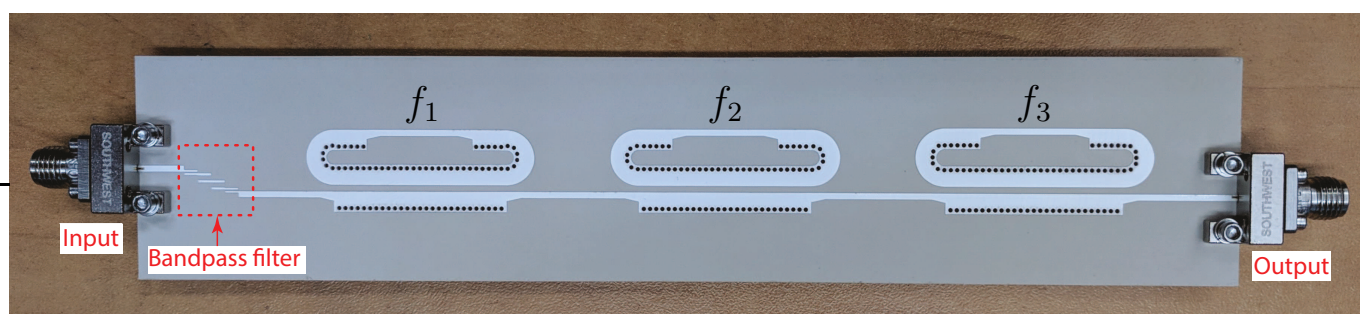

(b)

Figure 3.17: Photographs of the fabricated a) single and b) cascaded passive phasers with the bandpass filter at the input.

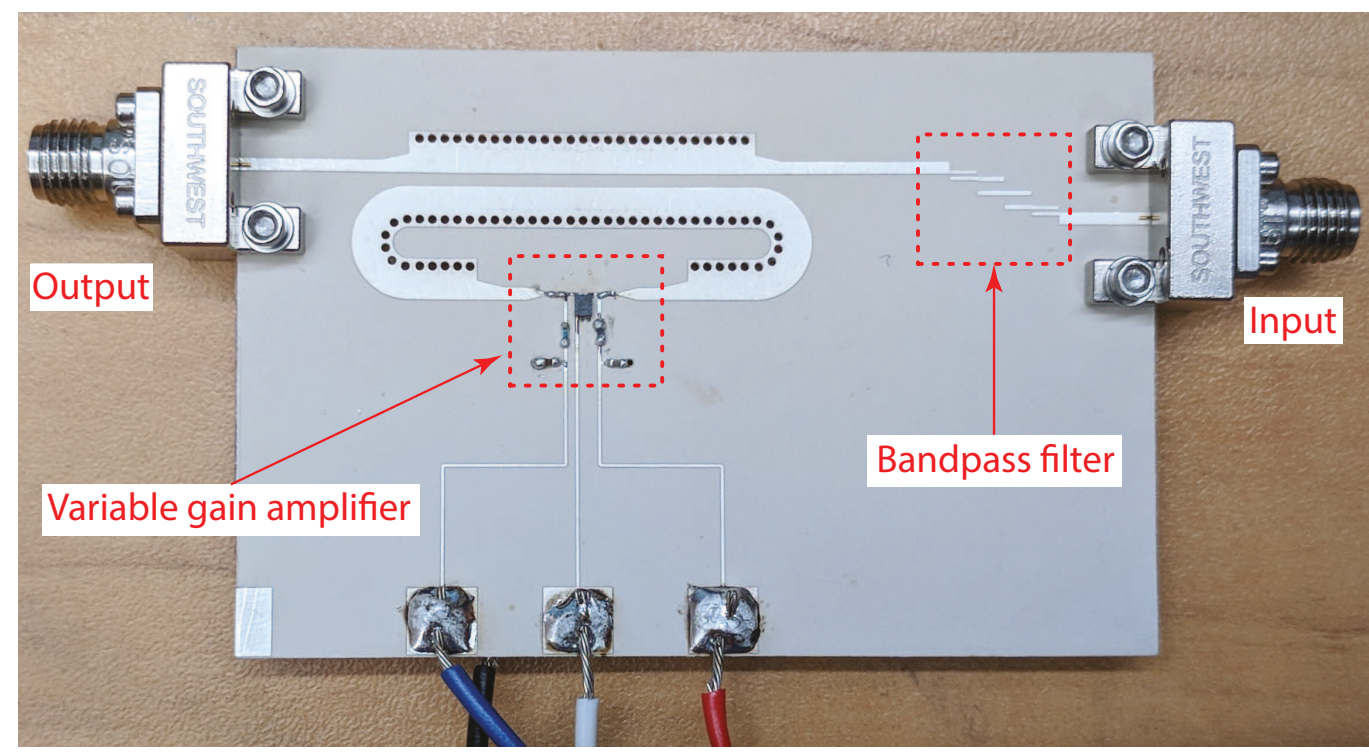

Figure 3.18: Photograph of the fabricated single active phaser with the amplifier integrated in the ring and the bandpass filter at the input. 


\subsubsection{Testing}

The S-parameters of the filter, amplifier, and phasers were measured using a Keysight 8722ES VNA (50 MHz to $40 \mathrm{GHz}$ ) at Carleton University. The VNA was calibrated using a standard SOLT calibration kit.

\subsubsection{Results}

Fig. 3.19 shows the experimental and FEM-HFSS simulated results for the bandpass filter. It is seen that while there is strong agreement between measurements and simulations, the responses shifted to the right in the measurements. This shift can be attributed to the difference between the dielectric constant of the substrate used in the simulations and that used in fabrication. It is imperative for the filter to have a flat group delay response to ensure minimal effect on the phaser group delay response. Overall, the filter exhibits acceptable performance with a nearly flat transmission and group delay responses in the operational bandwidth of the phaser. Moreover, the filter has good input and output reflection with responses greater than $10 \mathrm{~dB}$ in the operational bandwidth.

Fig. 3.20 and Fig. 3.21 show the experimental and FEM-HFSS simulated results for the single and cascaded passive phasers. Again, there is a strong agreement between the measurements and simulations, with a shift in the response which can be attributed to dielectric constant tolerances.

The single phaser exhibits more dissipation losses than predicted by the simulations, showing more than $25 \mathrm{~dB}$ transmission loss, and the group delay was slightly higher than predicted. In addition, the single passive phaser has acceptable input and output reflection responses greater than $10 \mathrm{~dB}$ in the operational bandwidth.

The cascaded passive phaser exhibits less, but still significant, dissipation losses than predicted by the simulations, showing more than $30 \mathrm{~dB}$ transmission loss, and the group delay was about 2 ns less than predicted. The cascaded passive phaser also has acceptable input and output reflection responses greater than $10 \mathrm{~dB}$ in a significant portion of the bandwidth.

The amplifier characterization (Fig. 3.15) and experimental demonstration of the active phaser (Fig. 3.18) is currently under development. 


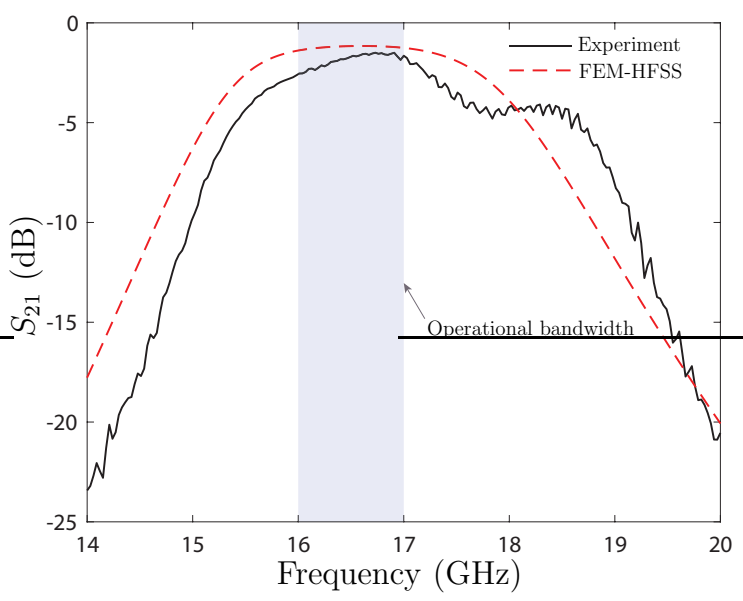

(a)

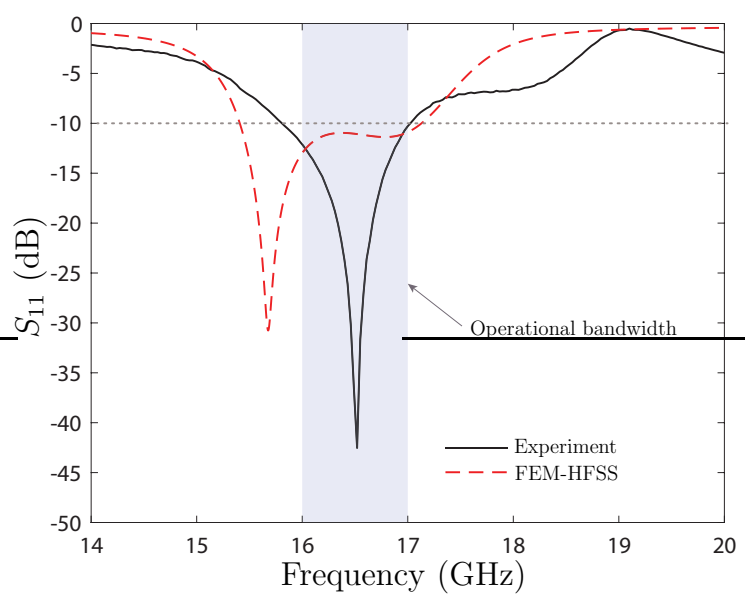

(c)

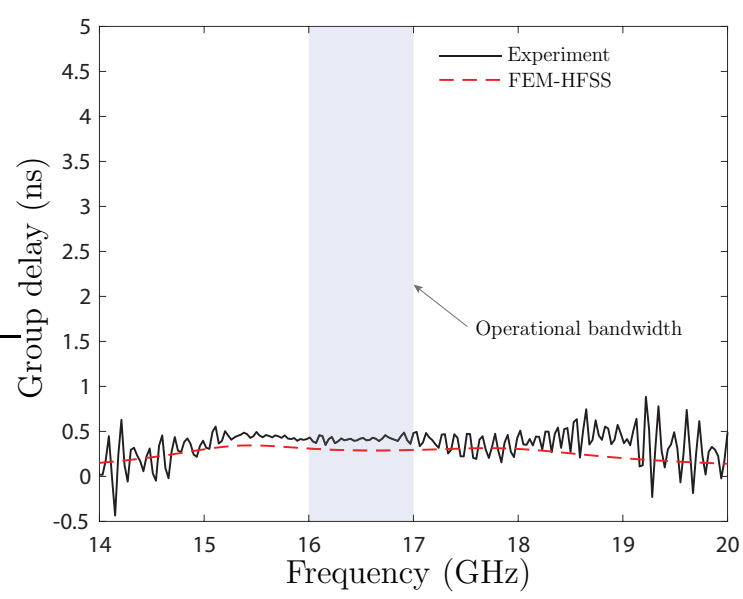

(b)

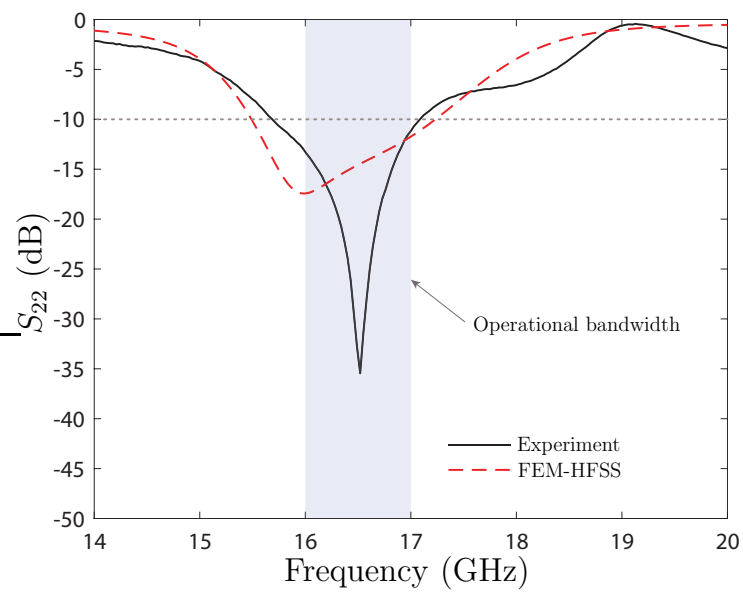

(d)

Figure 3.19: Bandpass filter results: a) transmission $\left(S_{21}\right)$, b) group delay, c) input return loss $\left(S_{11}\right)$, and d) output return loss $\left(S_{22}\right)$. 


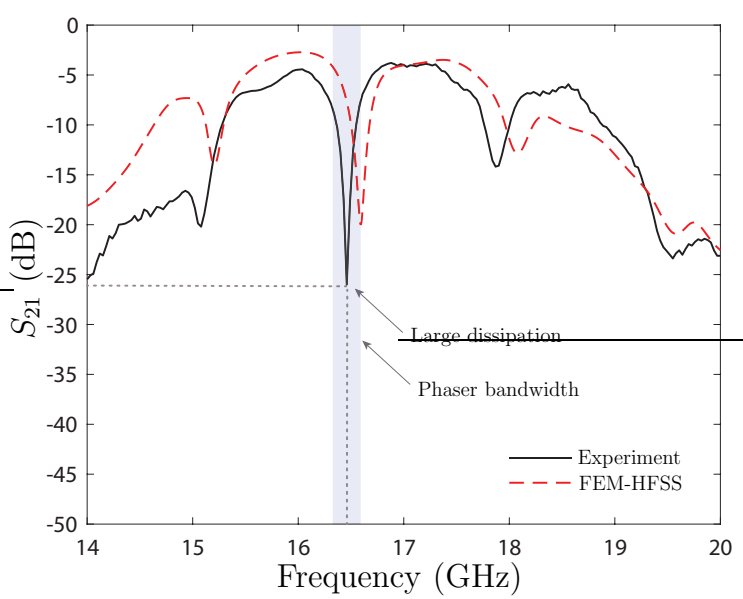

(a)

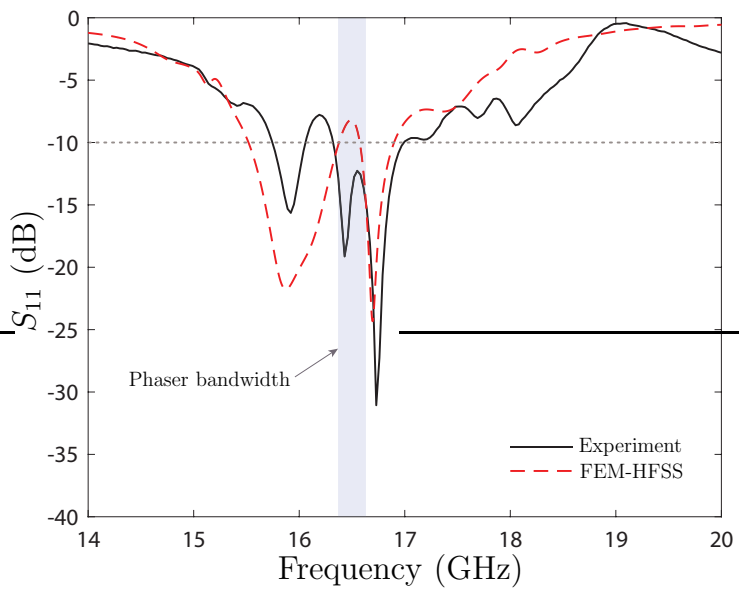

(c)

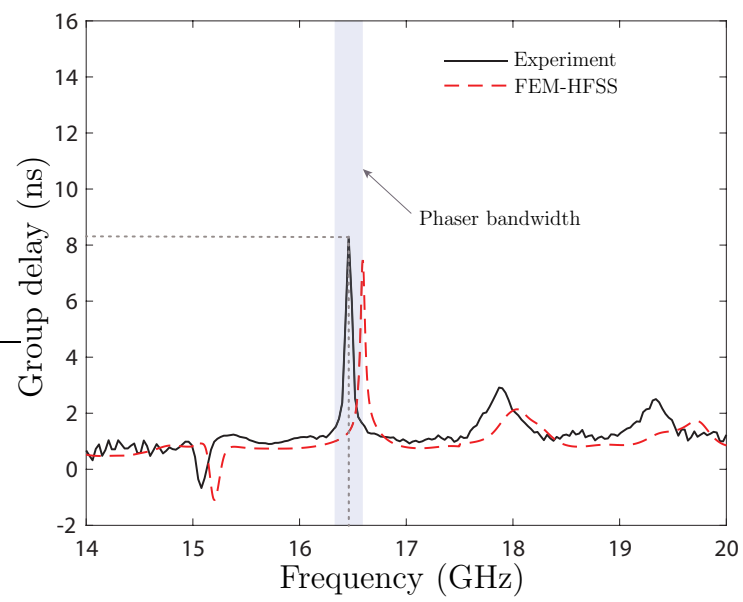

(b)

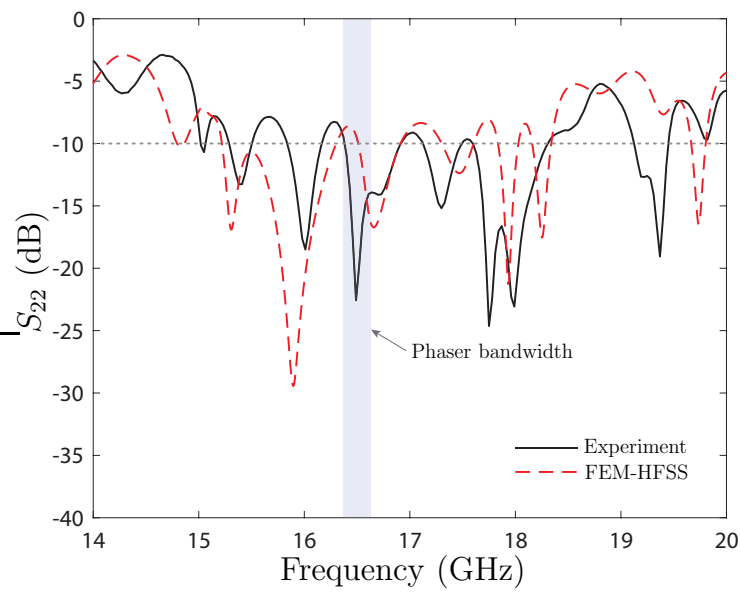

(d)

Figure 3.20: Single passive phaser results: a) transmission $\left(S_{21}\right)$, b) group delay, c) input return loss $\left(S_{11}\right)$, and d) output return loss $\left(S_{22}\right)$. 


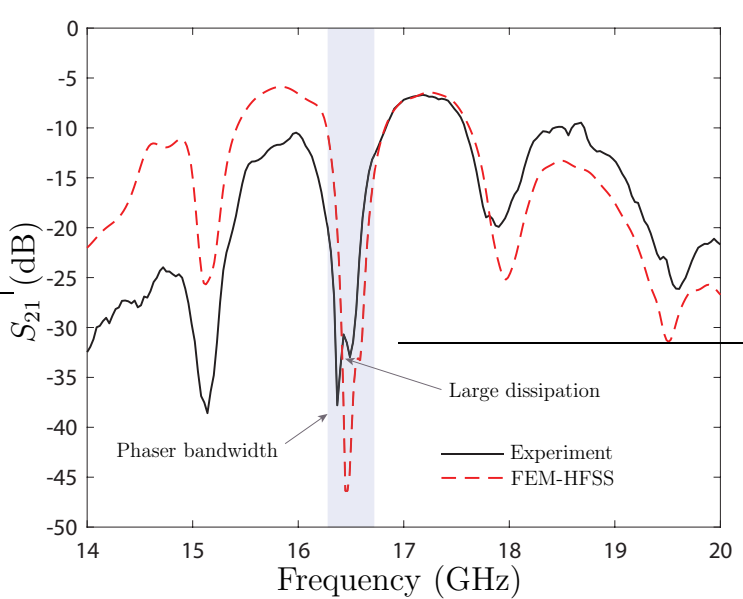

(a)

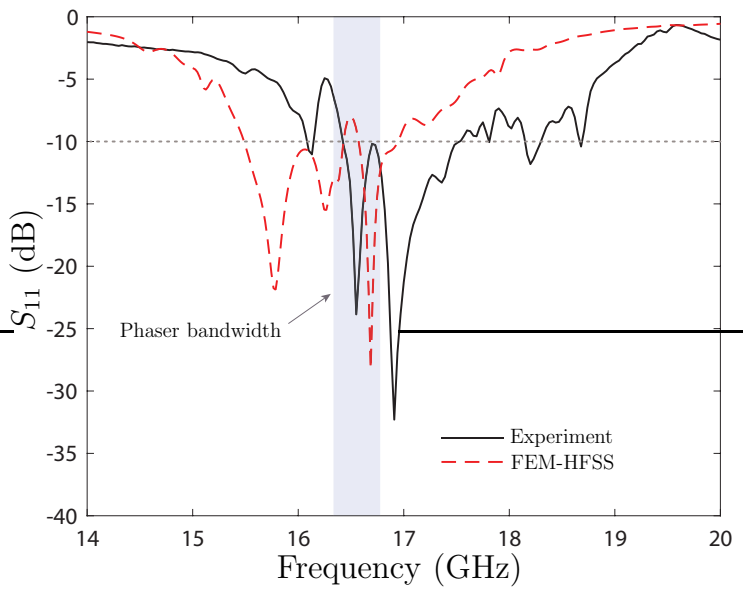

(c)

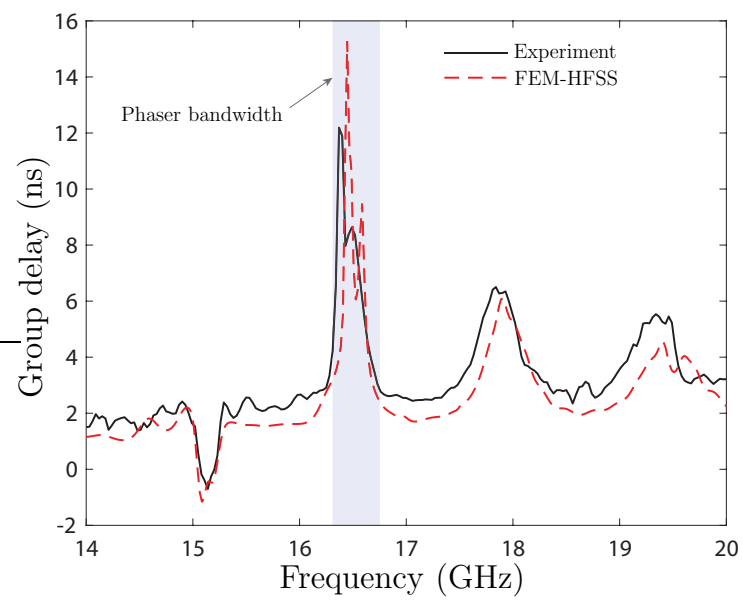

(b)

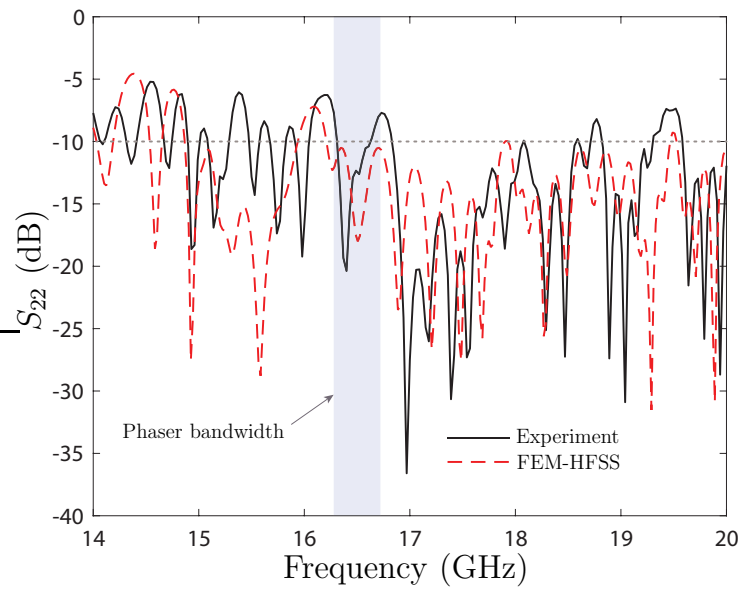

(d)

Figure 3.21: Cascaded passive phaser results: a) transmission $\left(S_{21}\right)$, b) group delay, c) input return loss $\left(S_{11}\right)$, and d) output return loss $\left(S_{22}\right)$. 


\subsection{Conclusion}

Active phasers based on co- and contra-directional couplers have been proposed for achieving a flat magnitude transmission while preserving their strong dispersive group delay response. The first proposed device is a microwave phaser based on a C-section for low frequency applications. The second phaser is based on micro-ring resonators and was implemented at $16.5 \mathrm{GHz}$ using HMSIW structures. Due to the non-TEM nature of the micro-ring phaser, it is ideally suitable for $\mathrm{mm}$-wave frequency operation, which is an important requirement in designing high-performance analog signal processing systems at these frequencies. The proposed phasers have been successfully demonstrated using full-wave simulations. These high performance phasers are critical in successful demonstration of processing mm-wave signals in real-time. 


\section{Chapter 4}

\section{Conclusions \& Future Work}

\subsection{Conclusions}

This thesis has presented two novel dispersion engineered electromagnetic structures for radiative and guided-wave applications. First, a low-profile grooved dielectric choke ring with dual-band surface impedance resonance has been proposed and experimentally demonstrated. The proposed structure is based on engineering the surface impedance of the dielectric based choke ring by strategically introducing two resonant frequencies around the GNSS bands of L2 and L1. This has been shown to provide capacitive surface impedances around both bands simultaneously, required for efficient surface wave suppression for higher multipath rejection performance. An in-house choke ring prototype of the proposed design has been tested with the DM antenna and has shown high multipath rejection performances in both GNSS bands, comparable to an otherwise bulky, heavy, and expensive conventional metallic choke ring. The proposed design further provides $58 \%$ reduction in height and $26 \%$ reduction in diameter using the FR4 material employed, and is an attractive low-cost solution for high performance GNSS applications. Second, active phasers based on co- and contra-directional couplers have been proposed for achieving a flat magnitude transmission while preserving their strong dispersive group delay response. The first proposed device is a microwave phaser based on a C-section for low frequency applications. The second phaser is based on micro-ring resonators and was implemented at $16.5 \mathrm{GHz}$ using HMSIW structures. Due to the non-TEM nature of the micro-ring phaser, it is ideally suitable for mm-wave frequency operation, which is an important requirement in designing high-performance analog signal processing systems at these frequencies. The proposed phasers have been successfully demonstrated using fullwave simulations. The experimental demonstration of the proposed active micro-ring phaser is currently under development. 


\subsection{Future Work}

\subsubsection{Dual-Band Dielectric Choke Ring GNSS Applications}

Future research on choke rings could potentially involve the following topics:

- A systematic design procedure, rather than full-wave simulations, involving an eigenmode analysis of the ground plane to produce dispersion diagrams of the propagation of surface waves to rigorously demonstrate the electromagnetic bandgap at L2 and L1 for the dual-band dielectric choke ring. This could be done using GA/FDTD [107], [108] or FEM [34], [56] models. It could also be experimentally demonstrated using various methods presented in literature. For example, using two monopole antennas at opposite ends of the surface and measuring the transmission response [34]. TM surface waves can be measured by orienting the antennas vertically, and TE surface waves can be measured by orienting the antennas horizontally. Another method is to use a suspended microstrip line over the choke ring and measure the transmission response as a function of frequency as was done in [50], [109].

- Different prototype implementations of the design, for example, using standard PCB processes and continuous copper plating instead of copper nails. A fully fabricated prototype, as opposed the in-house prototype presented here, will potentially have better performance and show even better agreement with the simulation results. Research could also explore other dielectrics which are less lossy and less dense than FR4, which will result in improved performance and a lighter structure.

- Exploration of 3D printing the conventional choke ring and copper platting the outside. This has the potential to produce a lighter and cheaper choke ring with theoretically the same performance of the fully metallic conventional choke ring. More investigation would need to be done into the mechanical integrity of the 3D-printed structure compared to that of the conventional choke ring.

- Exploration of making a multi-band design with various dimensions of grooves in the dielectric to create additional surface impedance resonances.

\subsubsection{Active Phasers for Analog Signal Processing Applica- tions}

Work is currently underway to experimentally demonstrate the active single and cascaded phasers using a VGA integrated inside the phaser ring. Future research on active phasers could potentially involve the following topics: 
- Implementation of phaser at higher frequencies close to the mm-wave band (e.g. $60 \mathrm{GHz}$ ) and hence make the phaser directly implementable in ASP applications for $5 \mathrm{G}$ networks. This work could potentially involve a fully integrated circuit design of the amplifier and the phaser on a silicon wafer.

- Implementation of a phaser with real-time dispersion control. The structure proposed here has a static dispersion response, but it could be made tunable using phase shifters inside the ring in addition to the amplifier. Phase shifters will allow tuning of the resonant frequency and group delay. This will allow realtime tuning of the dispersion characteristics for a wide range of frequencies and group delay dispersion responses (e.g. linearly decreasing, linearly increasing, parabolic, etc).

- Using the phaser to implement RTFT analog devices for real-time analysis of impulse signals [110] and real-time dispersion code multiple access (DCMA) [111] [112] for 5G applications. 


\section{Bibliography}

[1] M. Born and E. Wolf, Principles of Optics: Electromagnetic Theory of Propagation, Interference and Diffraction of Light. Cambridge University Press, 1999.

[2] B. E. A. Saleh and M. C. Teich, Fundamentals of Photonics. Wiley-Interscience, second ed., 2007.

[3] M. Sadiku, Elements of Electromagnetics. New York: Oxford University Press, 6 ed., 2015.

[4] D. V. Thiel, "On measuring electromagnetic surface impedance - discussions with Professor James R. Wait," IEEE Trans. Antennas Propagat., vol. 48, pp. 1517-1520, Oct. 2000.

[5] M. K. Emara, J. Hautcoeur, G. Panther, J. S. Wight, and S. Gupta, "Surface impedance engineered low-profile dual-band grooved-dielectric choke ring for GNSS applications," IEEE Trans. Antennas Propagat., 2018.

[6] M. K. Emara, S. Gupta, J. Hautcoeur, G. Panther, and J. S. Wight, "A lowprofile dual-band tunable AMC structure for GNSS antennas and its performance trade-offs," in 2018 18th International Symposium on Antenna Technology and Applied Electromagnetics (ANTEM), Aug. 2018.

[7] M. K. Emara, K. Maheshwari, J. S. Wight, and S. Gupta, "Amplitude-equalized microwave phasers," in International Applied Computational Electromagnetics Society (ACES) Symposium, Mar. 2018.

[8] M. K. Emara, J. S. Wight, and S. Gupta, "Active phasers based on codirectional couplers for millimeter-wave analog signal processing," in 2018 18th International Symposium on Antenna Technology and Applied Electromagnetics (ANTEM), Aug. 2018.

[9] "Department of Defense." https://www.gps.gov/governance/agencies/ defense/. Accessed: 2018-02-20.

[10] "An Introduction to GNSS." https://www.novatel.com/ an-introduction-to-gnss/chapter-3-satellite-systems/glonass/. Accessed: 2018-02-20. 
[11] "What is Galileo?." http://www.esa.int/Our_Activities/Navigation/ Galileo/What_is_Galileo. Accessed: 2018-02-20.

[12] E. Kaplan and C. Hegarty, Understanding GPS: Principles and Applications. Artech House mobile communications series, Artech House, 2006.

[13] F. Dovis, GNSS Interference Threats and Countermeasures. Norwood, MA: Artech House, 2015.

[14] W. E. McKinzie, R. B. Hurtado, B. K. Klimczak, and J. D. Dutton, "Mitigation of multipath through the use of an artificial magnetic conductor for precision GPS surveying antennas," in IEEE Antennas and Propagation Society International Symposium (IEEE Cat. No.02CH37313), vol. 4, pp. 640-643, 2002.

[15] J. Hao and S. Guo, "The study of dual frequency ionospheric error correction method and accuracy analysis based on GPS," in 2011 IEEE International Conference on Signal Processing, Communications and Computing (ICSPCC), pp. 1-4, 2011.

[16] D. Skournetou and E. S. Lohan, "Ionosphere-corrected range estimation in dual frequency global navigation satellite systems receivers," IET Radar, Sonar Navigation, vol. 5, pp. 215-224, Mar. 2011.

[17] "Inmarsat antennas." https://www.wirelessdesignonline.com/doc/ inmarsat-antennas-0001. Accessed: 2018-02-20.

[18] M. Berardo, E. G. Manfredini, F. Dovis, and L. L. Presti, "A spoofing mitigation technique for dynamic applications," in 2016 8th ESA Workshop on Satellite Navigation Technologies and European Workshop on GNSS Signals and Signal Processing (NAVITEC), pp. 1-7, Dec. 2016.

[19] F. Wang, H. Li, and M. Lu, "GNSS spoofing countermeasure with a single rotating antenna," IEEE Access, vol. 5, pp. 8039-8047, 2017.

[20] P. Y. Montgomery, T. E. Humphreys, and L. B. M., "Receiver-autonomous spoofing detection: Experimental results of a multi-antenna receiver defense against a portable civil GPS spoofer," in Proceedings of the 2009 International Technical Meeting of The Institute of Navigation, pp. 124-130, Jan. 2009.

[21] R. Baggen, M. Martinez-Vazquez, J. Leiss, S. Holzwarth, L. S. Drioli, and P. de Maagt, "Low profile GALILEO antenna using EBG technology," IEEE Trans. Antennas Propagat., vol. 56, pp. 667-674, Mar. 2008.

[22] J. M. Baracco, L. Salghetti-Drioli, and P. de Maagt, "AMC low profile wideband reference antenna for GPS and GALILEO systems," IEEE Trans. Antennas Propagat., vol. 56, pp. 2540-2547, Aug. 2008.

[23] P. Misra and P. Enge, Global Positioning System: Signals, Measurements, and Performance. Lincoln, MA: Ganga-Jammuna Press, 2012. 
[24] M. Maqsood, S. Gao, T. Brown, and M. Unwin, "Effects of ground plane on the performance of multipath mitigating antennas for GNSS," in 2010 Loughborough Antennas Propagation Conference, pp. 241-244, Nov. 2010.

[25] J. M. Tranquilla, J. P. Carr, and H. M. Al-Rizzo, "Analysis of a choke ring groundplane for multipath control in global positioning system (GPS) applications," IEEE Trans. Antennas Propagat., vol. 42, pp. 905-911, Jul. 1994.

[26] F. Scire-Scappuzzo and S. N. Makarov, "A low-multipath wideband GPS antenna with cutoff or non-cutoff corrugated ground plane," IEEE Trans. Antennas Propagat., vol. 57, pp. 33-46, Jan. 2009.

[27] R. E. Collin, Field Theory of Guided Waves. York, PA: McGraw-Hill, 1 ed., 1960.

[28] W. E. McKinzie, R. B. Hurtado, and B. K. Klimczak, "Artificial magnetic conductor technology reduces size and weight for precision GPS antennas," in Proceedings of the 2002 National Technical Meeting of The Institute of Navigation, vol. 4, pp. 448-459, Jan. 2002.

[29] F. Zhu, J. Xu, and Q. Xu, "Reduction of mutual coupling between closelypacked antenna elements using defected ground structure," in 2009 3rd IEEE International Symposium on Microwave, Antenna, Propagation and EMC Technologies for Wireless Communications, pp. 1-4, Oct. 2009.

[30] W. L. Barnes, T. W. Preist, S. C. Kitson, and J. R. Sambles, "Physical origin of photonic energy gaps in the propagation of surface plasmons on gratings," Phys. Rev. B, vol. 54, pp. 6227-6244, Sept. 1996.

[31] A. F. Harvey, "Periodic and guiding structures at microwave frequencies," IRE Transactions on Microwave Theory and Techniques, vol. 8, pp. 30-61, Jan. 1960.

[32] W. Rotman, "A study of single-surface corrugated guides," Proceedings of the IRE, vol. 39, pp. 952-959, Aug. 1951.

[33] C. Elachi, "Waves in active and passive periodic structures: A review," Proceedings of the IEEE, vol. 64, pp. 1666-1698, Dec. 1976.

[34] D. Sievenpiper, L. Zhang, R. F. J. Broas, N. G. Alexopolous, and E. Yablonovitch, "High-impedance electromagnetic surfaces with a forbidden frequency band," IEEE Trans. Microw. Theory Tech., vol. 47, pp. 2059-2074, Nov. 1999.

[35] R. E. Collin and F. J. Zucker, Antenna Theory, Part II. Inter-University Electronics Series, McGraw-Hill, 1969.

[36] D. Pozar, Microwave Engineering. Wiley, 3 ed., 2004. 
[37] C. Balanis, Antenna Theory: Analysis, and Design. New York: Wiley, 1 ed., 1982.

[38] D. Sievenpiper and E. Yablonovitch, "Circuit and method for eliminating surface currents on metals," US Patent 6,262,495, Jul. 2001.

[39] E. Yablonovitch, "Photonic band-gap structures," J. Opt. Soc. Am. B, vol. 10, pp. 283-295, Feb. 1993.

[40] F.-R. Yang, K.-P. Ma, Y. Qian, and T. Itoh, "A novel TEM waveguide using uniplanar compact photonic-bandgap (UC-PBG) structure," IEEE Trans. Microw. Theory Tech., vol. 47, pp. 2092-2098, Nov. 1999.

[41] A. Aminian, F. Yang, and Y. Rahmat-Samii, "In-phase reflection and EM wave suppression characteristics of electromagnetic band gap ground planes," in IEEE Antennas and Propagation Society International Symposium. Digest. Held in conjunction with: USNC/CNC/URSI North American Radio Sci. Meeting (Cat. No.03CH37450), vol. 4, pp. 430-433 vol.4, Jun. 2003.

[42] X. L. Bao, G. Ruvio, and M. J. Ammann, "Low-profile dual-frequency GPS patch antenna enhanced with dual-band EBG structure," Microw. Opt. Technology Lett., vol. 49, pp. 2630-2634, Nov. 2007.

[43] V. K. Pandit and A. R. Harish, "Dual-band monopole antenna loaded with dual-band AMC for WLAN/WiMAX applications," in 2016 International Conference on Emerging Trends in Communication Technologies (ETCT), pp. 1-3, Nov. 2016.

[44] S. X. Ta and I. Park, "Dual-band low-profile crossed asymmetric dipole antenna on dual-band AMC surface," IEEE Antennas Wirel. Propagat. Lett., vol. 13, pp. 587-590, 2014.

[45] L. M. Abdelghani, T. A. Denidni, and M. Niroo-Jazi, "Antenna beam shaping using conformal hybrid AMC/EBG reflectors," in 2014 IEEE Antennas and Propagation Society International Symposium (APSURSI), pp. 1770-1771, Jul. 2014.

[46] K. L. Ford and J. M. Rigelsford, "Street furniture antenna radiation pattern control using AMC surfaces," IEEE Trans. Antennas Propagat., vol. 56, no. 9, pp. 3049-3052, 2008.

[47] M. G. Bray and D. H. Werner, "A novel design approach for an independently tunable dual-band EBG AMC surface," in IEEE Antennas and Propagation Society Symposium, 2004., vol. 1, pp. 289-292 Vol.1, Jun. 2004.

[48] S. Makino, H. Hayashi, T. Moroya, K. Itoh, K. Noguchi, and T. Hirota, "Dual band characteristics of a linear antenna over a capacitance grid type AMC substrate," in 2014 IEEE-APS Topical Conference on Antennas and Propagation in Wireless Communications (APWC), pp. 93-96, Aug. 2014. 
[49] Y. Ge and K. P. Esselle, "Design of single-band and dual-band AMC surfaces by combining a micro-genetic algorithm with the spectral FDTD method," in 2006 IEEE Antennas and Propagation Society International Symposium, pp. 38533856, Jul. 2006.

[50] H. R. Cheng, Q. Song, Y.-C. Guo, X.-Q. Chen, and X.-W. Shi, "Design of a novel EBG structure and its application in fractal microstrip antenna," Progress In Electromagnetics Research C, vol. 11, pp. 81-90, 2009.

[51] J. H. Yoon, E. Y. Kim, Y. Lim, and Y. J. Yoon, "Equivalent circuit model and reflection phase control methods for dual-band AMC," in Proceedings of the 5th European Conference on Antennas and Propagation (EUCAP), pp. 1222-1226, Apr. 2011.

[52] P. S. Kildal, "Artificially soft and hard surfaces in electromagnetics," IEEE Trans. Antennas Propagat., vol. 38, pp. 1537-1544, Oct. 1990.

[53] L. R. Weill, "Conquering multipath: The GPS accuracy battle," GPS World: News and Applications of the Global Positioning System, vol. 8, pp. 59-66, Apr. 1997.

[54] L. Wanninger and M. May, "Carrier-phase multipath calibration of GPS reference stations," Navigation, vol. 48, no. 2, pp. 112-124, 2001.

[55] C. J. Comp and P. Axelrad, "Adaptive SNR-based carrier phase multipath mitigation technique," IEEE Trans. Aerosp. Electron. Syst., vol. 34, pp. 264276, Jan. 1998.

[56] C. C. Tchapwou and T. Bertuch, "Investigation of EBG surface performance for high-precision GPS applications," Electron. Lett., vol. 43, pp. 1327-1329, Nov. 2007.

[57] H. R. Khaleel, H. Al-Rizzo, A. Isaac, and A. Bihnam, "Multipath mitigation in high precision GPS systems using artificial magnetic conductors," in 2014 IEEE Antennas and Propagation Society International Symposium (APSURSI), pp. 432-433, Jul. 2014.

[58] S. N. Makarov and F. Scire-Scappuzzo, "Non-cutoff frequency selective surface ground plane antenna assembly," US Patent 8,451,190, May 2013.

[59] J. Ashjaee, V. S. Filippov, D. V. Tatarnikov, A. V. Astakhov, and I. V. Sutjagin, "Dual-frequency choke-ring ground planes," US Patent 6,278,407, Aug. 2001.

[60] V. Filippov, D. Tatarnicov, J. Ashjaee, A. Astakhov, and I. Sutiagin, "The first dual-depth dual-frequency choke ring," in Proceedings of the 11th International Technical Meeting of the Satellite Division of The Institute of Navigation (ION GPS 1998), pp. 1035-1040, 1998. 
[61] Y. Lee, S. Ganguly, and R. Mittra, "Multi-band L5-capable GPS antenna with reduced backlobes," in 2005 IEEE Antennas and Propagation Society International Symposium, vol. 1A, pp. 438-441 Vol. 1A, Jul. 2005.

[62] Y. Lee, S. Ganguly, and R. Mittra, "Multifrequency antenna with reduced rear radiation and reception," US Patent 6,940,457, 2005.

[63] L. I. Basilio, R. L. Chen, J. T. Williams, and D. R. Jackson, "A new planar dualband GPS antenna designed for reduced susceptibility to low-angle multipath," IEEE Trans. Antennas Propagat., vol. 55, pp. 2358-2366, Aug. 2007.

[64] L. I. Basilio, J. T. Williams, D. R. Jackson, and M. A. Khayat, "A comparative study of a new GPS reduced-surface-wave antenna," IEEE Antennas Wirel. Propagat. Lett., vol. 4, pp. 233-236, 2005.

[65] D. R. Jackson, J. T. Williams, A. K. Bhattacharyya, R. L. Smith, S. J. Buchheit, and S. A. Long, "Microstrip patch designs that do not excite surface waves," IEEE Trans. Antennas Propagat., vol. 41, pp. 1026-1037, Aug. 1993.

[66] L. Boccia, G. Amendola, and G. D. Massa, "A dual frequency microstrip patch antenna for high-precision GPS applications," IEEE Antennas Wirel. Propagat. Lett., vol. 3, pp. 157-160, 2004.

[67] L. Boccia, G. Amendola, G. D. Massa, and L. Giulicchi, "Shorted annular patch antennas for multipath rejection in GPS-based attitude determination systems," Microw. Opt. Technology Lett., vol. 28, no. 1, pp. 47-51, 2001.

[68] L. Boccia, G. Amendola, and G. D. Massa, "A shorted elliptical patch antenna for GPS applications," IEEE Antennas Wirel. Propagat. Lett., vol. 2, pp. 6-8, 2003.

[69] W. Kunysz, "High performance GPS pinwheel antenna," tech. rep., NovAtel Inc.

[70] L. Bedford, N. Brown, and J. Walford, "Leica AR25 White Paper," tech. rep., Leica Geosystems AG, Feb. 2009.

[71] D. Tatarnikov, "PN-A5: Topcon full wave reference station antenna with convex impedance ground plane," tech. rep., Topcon Positioning Systems Inc.

[72] S. H. Huynh and G. Cheng, "Low profile ceramic choke," US Patent 6,040,805, Mar. 2000.

[73] W. EnCheng, W. Zhuopeng, and C. Zhang, "A wideband antenna for global navigation satellite system with reduced multipath effect," IEEE Antennas Wirel. Propagat. Lett., vol. 12, pp. 124-127, 2013.

[74] N. A. Abbasi and R. Langley, "A wideband printed monopole antenna over dualband AMC," in 2010 Loughborough Antennas Propagation Conference, pp. 221224, Nov. 2010. 
[75] E. Y. Kim, J. H. Yoon, Y. J. Yoon, and C. G. Kim, "Low profile dual-band reflector antenna with dual resonant AMC," in 2011 IEEE International Symposium on Antennas and Propagation (APSURSI), pp. 1800-1803, Jul. 2011.

[76] E. R. Gafarov and Y. P. Salomatov, "Hexagonal FSS for GLONASS/GPS antenna with improved axial ratio," in 2011 International Siberian Conference on Control and Communications (SIBCON), pp. 159-161, 2011.

[77] N. Chahat, L. R. Amaro, J. Harrell, C. Wang, P. Estabrook, and S. A. Butman, "X-band choke ring horn telecom antenna for interference mitigation on NASA's SWOT mission," IEEE Trans. Antennas Propagat., vol. 64, pp. 2075-2082, Jun. 2016.

[78] C. Francque and P. A. Lindsey, "Mobile platform window choke rings controlling electromagnetic interference with electronics systems," US Patent 2008/0218421, Sept. 2008.

[79] M. Mantash and A. C. Tarot, "On the bandwidth and geometry of dual-band AMC structures," in 2016 10th European Conference on Antennas and Propagation (EuCAP), pp. 1-4, Apr. 2016.

[80] F. Linot, R. Cousin, X. Begaud, and M. Soiron, "Design and measurement of high impedance surface," in Proceedings of the Fourth European Conference on Antennas and Propagation, pp. 1-4, Apr. 2010.

[81] R. K. Gupta, "On radiation properties of the alford loop and a dipole antenna," India, IEE-IERE Proceedings, vol. 9, pp. 145-149, Jul. 1971.

[82] M. Bozzi, A. Georgiadis, and K. Wu, "Review of substrate-integrated waveguide circuits and antennas," IET Microwaves, Antennas Propagation, vol. 5, pp. 909920, Jun. 2011.

[83] "Instrumentation." http://www.grames.polymtl.ca/facilities/ instrumentation/. Accessed: 2018-04-07.

[84] "VP6000." http://www.tallysman.com/index.php/gnss/products/ antennas-all-gnss-signals/vp6000/. Accessed: 2018-04-07.

[85] C. Caloz, S. Gupta, Q. Zhang, and B. Nikfal, "Analog signal processing: A possible alternative or complement to dominantly digital radio schemes," IEEE Microw. Mag., vol. 14, pp. 87-103, Sept. 2013.

[86] F. Boccardi, R. W. Heath, A. Lozano, T. L. Marzetta, and P. Popovski, "Five disruptive technology directions for 5G," IEEE Communications Magazine, vol. 52, pp. 74-80, Feb. 2014.

[87] T. S. Rappaport, S. Sun, R. Mayzus, H. Zhao, Y. Azar, K. Wang, G. N. Wong, J. K. Schulz, M. Samimi, and F. Gutierrez, "Millimeter wave mobile communications for 5G cellular: It will work!," IEEE Access, vol. 1, pp. 335-349, 2013. 
[88] S. Gupta and C. Caloz, "Perfect dispersive medium for real-time signal processing," IEEE Trans. Antennas Propagat., vol. 64, pp. 5299-5308, Dec. 2016.

[89] E. G. Cristal, "Theory and design of transmission line all-pass equalizers," IEEE Trans. Microw. Theory Tech., vol. 17, pp. 28-38, Jan. 1969.

[90] G. Matthaei, E. M. T. Jones, and L. Young, Microwave Filters, ImpedanceMatching Networks, and Coupling Structures. Artech House Publishers, 1980.

[91] S. Gupta, D. L. Sounas, Q. Zhang, and C. Caloz, "All-pass dispersion synthesis using microwave C-sections," Int. J. Circ. Theory Appl., May 2013.

[92] S. Gupta, Q. Zhang, L. Zou, L. J. Jiang, and C. Caloz, "Generalized coupledline all-pass phasers," IEEE Trans. Microw. Theory Tech., vol. 63, pp. 1007 1018, Mar. 2015.

[93] C. Caloz, S. Gupta, Q. Zhang, and B. Nikfal, "Analog signal processing," Microw. Mag., vol. 14, pp. 87-103, Sept. 2013.

[94] S. Gupta and C. Caloz, "Perfect dispersive medium," arXiv:1511.00671, Nov. 2015.

[95] L. Zou, S. Gupta, and C. Caloz, "Loss-gain equalized reconfigurable C-section analog signal processor," IEEE Trans. Microw. Theory Tech., vol. 65, pp. 555564, Feb. 2017.

[96] T. Jannson, "Real-time Fourier transformation in dispersive optical fibers," Opt. Lett., vol. 8, pp. 232-234, Apr. 1983.

[97] M. A. G. Laso, T. Lopetegi, M. J. Erro, D. Benito, M. J. Garde, M. A. Muriel, M. Sorolla, and M. Guglielmi, "Real-time spectrum analysis in microstrip technology," IEEE Trans. Microw. Theory Tech., vol. 51, pp. 705-717, Mar. 2003.

[98] B. Nikfal, S. Gupta, and C. Caloz, "Increased group delay slope loop system for enhanced-resolution analog signal processing," IEEE Trans. Microw. Theory Tech., vol. 59, pp. 1622-1628, Jun. 2011.

[99] S. Gupta, D. L. Sounas, H. V. Nguyen, Q. Zhang, and C. Caloz, "CRLHCRLH C-section dispersive delay structures with enhanced group delay swing for higher analog signal processing resolution," IEEE Trans. Microw. Theory Tech., vol. 60, pp. 3939-3949, Dec. 2012.

[100] S. Gupta, A. Parsa, E. Perret, R. V. Snyder, R. J. Wenzel, and C. Caloz, "Group delay engineered non-commensurate transmission line all-pass network for analog signal processing," IEEE Trans. Microw. Theory Tech., vol. 58, pp. 23922407, Sept. 2010.

[101] S. Gupta, "Active micro-ring resonators as compact perfect dispersive devices," in 2016 Photonics North (PN), pp. 1-1, May 2016. 
[102] J. Hirokawa and M. Ando, "Single-layer feed waveguide consisting of posts for plane tem wave excitation in parallel plates," IEEE Trans. Antennas Propagat., vol. 46, pp. 625-630, May 1998.

[103] F. Xu and K. Wu, "Guided-wave and leakage characteristics of substrate integrated waveguide," IEEE Trans. Microw. Theory Tech., vol. 53, pp. 66-73, Jan. 2005.

[104] M. Bozzi, A. Georgiadis, and K. Wu, "Review of substrate-integrated waveguide circuits and antennas," IET Microwaves, Antennas Propagation, vol. 5, pp. 909920, Jun. 2011.

[105] W. Hong, B. Liu, Y. Wang, Q. Lai, H. Tang, X. X. Yin, Y. D. Dong, Y. Zhang, and $\mathrm{K} . \mathrm{Wu}$, "Half mode substrate integrated waveguide: A new guided wave structure for microwave and millimeter wave application," in 2006 Joint 31st International Conference on Infrared Millimeter Waves and 14th International Conference on Teraherz Electronics, pp. 219-219, Sept. 2006.

[106] "MAAM-011100 Broadband Variable Gain Amplifier (VGA)." https://cdn . macom.com/datasheets/MAAM-011100.pdf. Accessed: 2018-04-08.

[107] Y. Ge and K. Esselle, "GA/FDTD technique for the design and optimisation of periodic metamaterials," IET Microw. Antennas Propagat., vol. 1, pp. 158-164, Feb. 2007.

[108] A. Aminian, F. Yang, and Y. Rahmat-Samii, "Bandwidth determination for soft and hard ground planes by spectral FDTD: a unified approach in visible and surface wave regions," IEEE Trans. Antennas Propagat., vol. 53, pp. 18-28, Jan. 2005.

[109] M. Y. Fan, R. Feng, Z. H. Hu, X. X. Zhang, and Q. Hao, "Advance in 2D-EBG research," Journal of Infrared Millimeter Waves, vol. 22, pp. 883-887, 2003.

[110] S. Gupta and C. Caloz, "Analog real-time Fourier transformer using a group delay engineered C-section all-pass network," in 2010 IEEE Antennas and Propagation Society International Symposium, pp. 1-4, Jul. 2010.

[111] L. Zou, S. Gupta, and C. Caloz, "Real-time dispersion code multiple access for high-speed wireless communications," IEEE Trans. Wirel. Commun., vol. 17, pp. 266-281, Jan. 2018.

[112] S. Gupta, L. Zou, M. A. Salem, and C. Caloz, "Bit-error-rate (BER) performance in dispersion code multiple access (DCMA)," in 2015 IEEE International Symposium on Antennas and Propagation USNC/URSI National Radio Science Meeting, pp. 1015-1016, Jul. 2015. 
Appendices 


\section{Appendix A}

\section{Dual-Band Choke Ring Drawing}

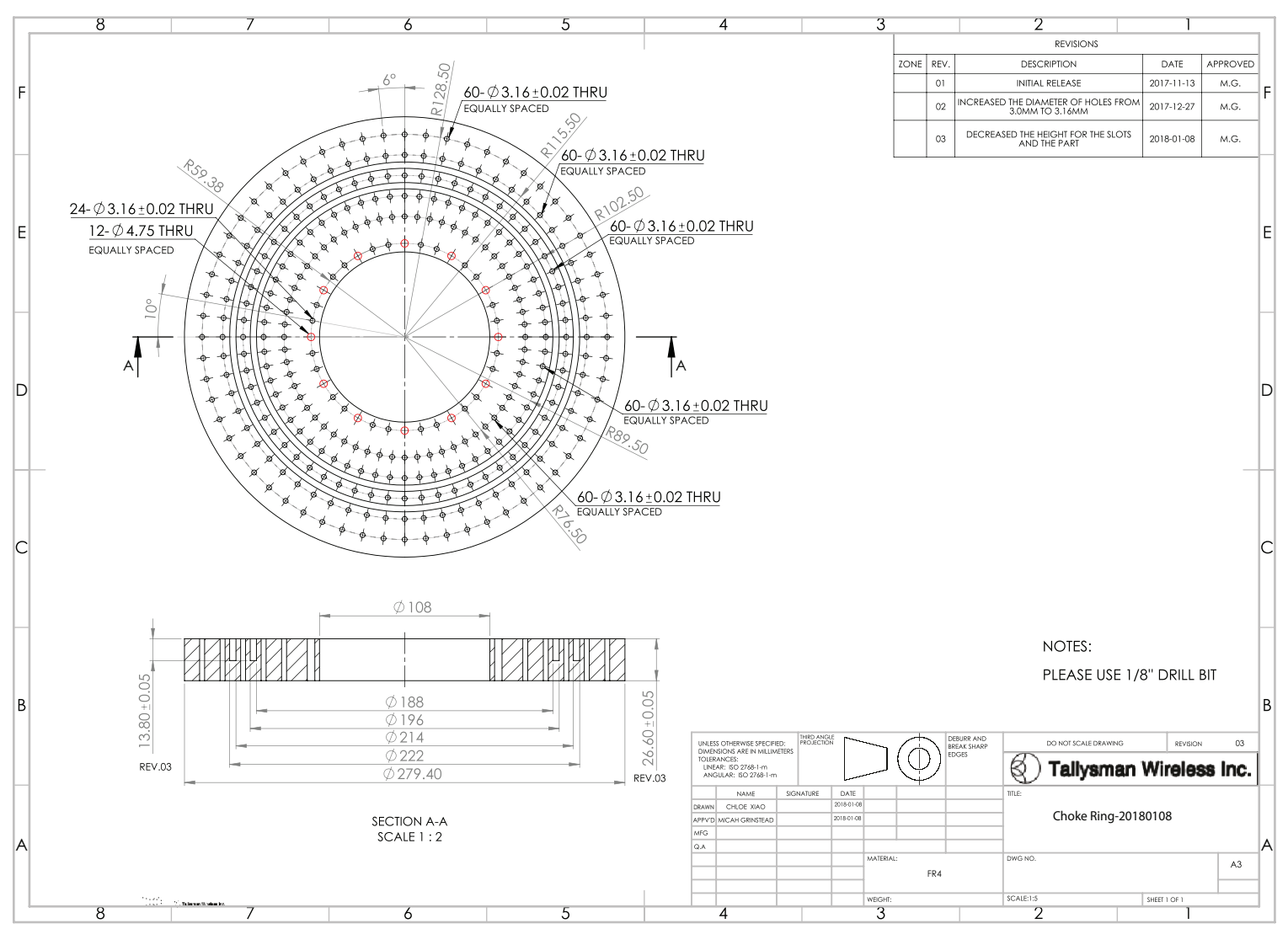

Figure A.1: Mechanical drawing of the manufactured dual-band dielectric choke ring 